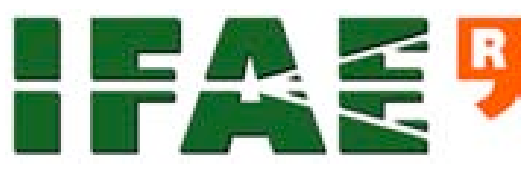

INSTITUT DE FÍSICA

D'ALTES ENERGIES
UAB

UNIVERSITAT AUTÒNOMA

DE BARCELONA

\title{
Measurement of the Inclusive Isolated Prompt Photon Production Cross Section at the Tevatron using the CDF Detector
}

\author{
PhD Dissertation \\ Carolina Deluca Silberberg \\ IFAE \\ Edifici Cn, UAB \\ 08193 Bellaterra (Barcelona) \\ <cdeluca@fnal.gov>
}

April 2009

supervised by:
Sebastian Grinstein
ICREA
and

Mario Martínez Pérez

IFAE / ICREA

Universitat Autònoma de Barcelona 

The results presented in this Thesis have been approved by the CDF Collaboration and will lead to a publication in Physics Review Letters. 



\section{Acknowledgments}

During this $\mathrm{PhD}$ thesis I have met great physicists, but also extraordinary people.

My greatest debt is with Raymond Culbertson, without whose guidance, advice and support this work would not have been possible. His expertise on photons has been the most valuable input for this measurement. He has been involved with my analysis before I moved to Fermilab, and I have learned many things from the useful discussions we had together. When I arrived to Fermilab Eiko joined us, and it is with her that I have my second greatest debt. Eiko has been always available and ready to help me with technical things, and together with Ray, followed my work day by day, and contributed with many useful studies for the measurement. I would like to thank them both for their help and great companionship, for the friendly environment in our meetings, for the discussions about physics, and for every day of working together. This result is as mine as theirs. My last greatest debt is with Sebastian Grinstein, with whom I have been working very closely since I came back to Barcelona. His questions and his rigor have been crucial for the final form of the analysis and of this thesis itself. I would like to thank him for his help and availability to discuss all the details of the analysis. Thanks for being my supervisor and friend.

This work would not have been possible without the support of Mario Martínez, who gave me the opportunity to join the CDF group at IFAE and encouraged me to move to Fermilab. I would like to thank him for his valuable advice and foresight. I also would like to thank Enrique Fernández for giving me the opportunity of joining the IFAE, and to Matteo Cavalli-Sforza, for his advice and help in the crucial moments. I would like to express my gratitude to Martine Bosman, my Master Thesis supervisor, for helping me in the very beginning of my learning process. Thanks to all of them for the freedom they always gave me to choose the experiment and my Thesis project.

There is other people from IFAE I must thank for. To Carlos, my first office-mate, and my first good friend at IFAE, thanks for being there. And to Mireia, for being also my first good friend at IFAE and for the good times with our daughters. I also want to mention Emili and Ramón, thanks for the funniest lunches ever and for your friendship. I missed you while I was at Fermilab. Thanks to Jordi, Lluís, Estel, Carlos Osuna, Sigrid, Xavi Espinal, John, and all the people at IFAE, for making of it a good place to stay. Special thanks to my IFAE CDF workmates and friends, for creating such a good environment to work. To Xavi, for your patience and help when I arrived at IFAE, and for being always so good. To Olga for all this time we shared at Fermilab. 
I do not forget Oriol, with his great sense of humor, his friendship and his unconditional help when I started working at CDF. To Gianluca, for his crazy solutions and his always funny point of view. And to Stefano, for the very deep analysis and conversations. To Monica, thanks for her unbiased approach to problems when helping me, and to Alon, for the good company in the office at Fermilab.

This work was done with the help of many people who did not contribute directly to the analysis but that enriched my life everyday. I would like to express my most sincere thanks to all the friends I met at Fermilab. My most warm memory is for Barbara, who supported me in everything even before I arrived at Fermilab. Thanks Barbara for all the times we had together. Another good friend is Cristobal, thanks for the good advice and the sincerity, for all the visits and for the great paellas. To Belén and Esteban, for keeping alive the paella tradition, and above all, for being so nice, so good and my friends. There were other friends there that I still miss, thanks Miguel for being so funny, and to Bruno for the good times before the bar was closed, and to Kike, thanks for being able to express a lot of things with so little words. Clearly, this is an ability I lack of. To Arán, thanks for the advice and his always good humor. And to Oscar, thanks for being always ready to help me. I had other good friends at Chicago I do not forget. To Ainara, thanks for the very funny times together. And to the Argonne people, Paola, M. Ángeles and Xavi, thanks for the Saturday lunches, the volleyball and the good times. To all of you, the biggest thanks for being as uncles and aunties to my daughter Maitane.

My family always supported and encouraged me. I would like to thank my parents, that took care of me even at $7.000 \mathrm{~km}$ distance, and to my sister Anita, who has been my best friend since we were kids. I thank Martin, my youngest brother, for his goodness, and Manuel for his jokes. To Djasmine, for the very especial times we had, and Laura, for her fighter nature. Finally, thanks to my eldest brother, Pablo, for the fantastic nights listening to your guitar. Thanks to you all for being such a big family. The family has grown larger with Dani, Ingrid, Amy, Cris and Ger, who also help (or will help) my brothers and sisters to give me the marvelous nice and nephews I have.

The last and most important words are for my little family. Thanks Ernest, for being my companion and best friend, and the best father Maitane and I could ever dream of. With his, my greatest thanks go to Maitane, my daughter, the little person who makes me happy every day. Without her, these days would not have been as funny, interesting, challenging and unforgettable as they have been. 


\section{Contents}

List of Figures $\quad$ v

List of Tables $\quad$ xiii

1 Introduction 1

2 Theory 3

2.1 Hadron Scattering Formalism . . . . . . . . . . . . . . . . . . . . . 3

2.2 Prompt photon production $\ldots \ldots \ldots \ldots \ldots$

2.2 .1 Isolated prompt photon calculations $\ldots \ldots \ldots . \ldots . \ldots$

2.2.2 Previous measurements . . . . . . . . . . . . . . . . . 11

2.3 Monte Carlo Event Generators . . . . . . . . . . . . . . . . . . . . 14

$2.3 .1 \quad$ PYTHIA Monte Carlo . . . . . . . . . . . . . . . . . . . . 15

3 The CDF Detector 17

3.1 The Tevatron . . . . . . . . . . . . . . . . . . . . 17

3.2 The CDF Run II Detector . . . . . . . . . . . . . . . . . . . . . . . . . . 19

3.2 .1 Tracking Systems . . . . . . . . . . . . . . . . . . 20

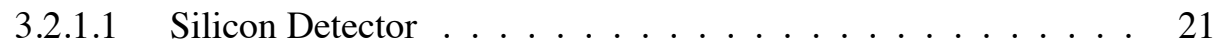

3.2.1.2 Central Outer Tracker . . . . . . . . . . . . . . . 22 


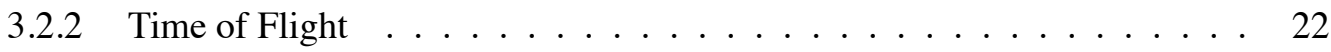

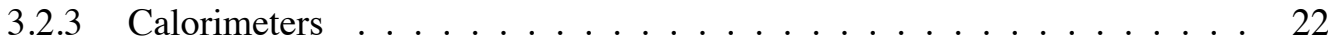

3.2.3.1 Central Calorimeters . . . . . . . . . . . . . . 23

3.2.3.2 Forward Calorimeters . . . . . . . . . . . . . 26

3.2.3.3 Shower Profile detectors . . . . . . . . . . . . . . . . 26

3.2.3.4 Pre-Radiator detectors . . . . . . . . . . . . . . 27

3.2.3.5 EMTiming system . . . . . . . . . . . . . 27

3.2 .4 Muon detectors . . . . . . . . . . . . . . . . . . . . . . 28

3.2 .5 Trigger and DAQ systems $\ldots \ldots \ldots \ldots$

3.2 .6 Luminosity measurement . . . . . . . . . . . . . . . . . . 31

3.3 Offline Photon Reconstruction . . . . . . . . . . . . . . . . . . . . . . . 32

4 The Inclusive Isolated Prompt Photon Cross Section Measurement 35

4.1 Data Samples . . . . . . . . . . . . . . . . . . . . . . 35

4.2 Monte Carlo Samples . . . . . . . . . . . . . . . . . . . . . . . . . 37

4.3 Event Selection . . . . . . . . . . . . . . . . . . . . . 37

4.3.1 Photon selection variables $\ldots \ldots \ldots 38$

4.3.2 Photon-like electron identification . . . . . . . . . . . . . . . 40

4.4 The photon energy scale $\ldots \ldots \ldots \ldots \ldots$. . . . . . . . . . . . . 41

4.5 Triggers Efficiency $\ldots \ldots \ldots \ldots$. . . . . . . . . . . . . . . 44

4.5.1 PHOTON_25_ISO trigger efficiency . . . . . . . . . . . . . . 44

4.5.2 SUPER_PHOTON70 trigger efficiency . . . . . . . . . . . . . 46

4.6 Background Subtraction $\ldots \ldots \ldots$. . . . . . . . . . . . . . . 46

4.6.1 Non-collision background . . . . . . . . . . . . . . . . . . 47

4.6 .2 Light meson background $\ldots \ldots \ldots$. . . . . . . . . . . 48

4.6.2.1 Isolation templates . . . . . . . . . . . . . . . . . 50

4.6.2.2 The signal template correction . . . . . . . . . . . 51 
4.6.2.3 Fit results .................. 53

4.7 Unfolding Factors . . . . . . . . . . . . . . . . . . 61

5 Systematic Uncertainties $\quad 65$

5.1 Systematic in the Signal Fractions . . . . . . . . . . . . . . . 65

5.1.1 Methods to estimate the systematic uncertainty . . . . . . . . . . 65

5.1 .2 The systematic uncertainty . . . . . . . . . . . . . 68

5.2 Systematics due to photon ID efficiency $\ldots \ldots \ldots \ldots \ldots$

5.2.1 The CES $\chi^{2}$ cut efficiency . . . . . . . . . . . . 70

5.2.2 Uncertainty in the photon acceptance . . . . . . . . . . . . 72

5.3 Systematics due to the Photon Energy Scale . . . . . . . . . . . . . . . 72

5.4 Systematics due to the Photon Isolation Scale . . . . . . . . . . . . . . 74

5.5 Other sources of systematics $\ldots \ldots \ldots \ldots \ldots \ldots$

5.6 Total Systematic Uncertainty . . . . . . . . . . . . . . . . . . . . 75

6 Results $\quad 79$

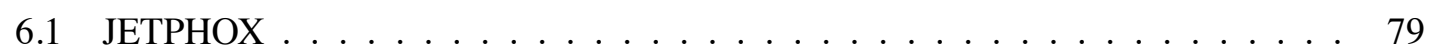

$6.1 .1 \quad$ PDF Uncertainty $\ldots \ldots \ldots \ldots$

6.1.2 Dependence on the renormalization, factorization and fragmentation scales 80

6.1.3 Non-pQCD contributions . . . . . . . . . . . . . . . . . . . 80

6.2 The Cross Section Result . . . . . . . . . . . . . . . . . . . . . . . . 82

$\begin{array}{lll}7 & \text { Conclusions } & 91\end{array}$

$\begin{array}{lll}A & \text { Fit results using } Z \text { templates } & 93\end{array}$

$\begin{array}{lll}\text { B Fit results using corrected dijet MC templates } & 97\end{array}$ 
$\begin{array}{ll}\text { C Study of the Isolation in the Photon Monte Carlo } & 105\end{array}$

C.1 Isolation at Raw, Leakage and Corrected levels _ . . . . . . . . . . . . . . 107

C.2 Underlying Event study using random cones . . . . . . . . . . . . . . . . . . 111

D Rewighting of the photon Monte Carlo 113

$\begin{array}{ll}\text { Bibliography } & 117\end{array}$ 


\section{List of Figures}

2.1 Summary of measurements of the strong coupling $\alpha_{s}\left(Q^{2}\right)$ as a function of the respective energy scale Q. The curves are the QCD predictions for the combined world average value of $\alpha\left(M_{Z}^{2}\right)$. Figure taken from [7]. . . . . . . . . . . . . . . . .

2.2 Diagram of the interaction between two hadrons. The process is described as the convolution of the Parton Density Functions (PDFs) of the partons inside the incoming hadrons. The hard scattering is described by the partonic cross section, $\sigma$, which can be calculated perturbatively. The outgoing partons can fragment into other particles. This process is taken into account by the fragmentation functions (Ds). . . . . . . . . . . . 5

2.3 Example of proton PDFs for $Q^{2}=1000 \mathrm{GeV}^{2}$ as given by CTEQ6.1M [9]. . . . . . . . 6

2.4 Prompt photon production diagrams. The two upper diagrams show the $q \bar{q}$ annihilation process $q \bar{q} \rightarrow g \gamma$. The second row of diagrams correspond to the Compton process $q(\bar{q}) g \rightarrow q(\bar{q}) \gamma$. The two diagrams in the bottom show two examples of fragmentation contributions. The left one is the point-like fragmentation of a quark into a photon, which can be calculated perturbatively for asymptotically large scales. The right one is the nonperturbative fragmentation of a gluon producing a photon. . . . . . . . . . . . .

2.5 Comparison of the JETPHOX perturbative QCD predictions at LO and NLO for central $(|\eta|<1)$ photons with $E_{T}^{\gamma}>30 \mathrm{GeV}$ and $E_{T}^{i s o}<2 \mathrm{GeV}$. . . . . . . . . . . . . .

2.6 Ratio data to theory for collider and fixed target data with scales $\mu=p_{T} / 2$. Measurements for fixed target data at low energies are inclusive, while DO and CDF data come from isolated measurements. Figure taken from [21].

3.1 The accelerator complex at FNAL. . . . . . . . . . . . . . . . . 18 
3.2 Isometric view of the CDF Run II detector with its main subsystems (innermost to outermost): the Silicon Vertex Detector (green), the Central Outer Tracker (orange), the superconducting solenoid (pink), the electromagnetic calorimeter (red), the hadronic calorimeter (blue) and the muon chamber (yellow and blue) . . . . . . . . . . . . . . 19

3.3 The CDF coordinate system. . . . . . . . . . . . . . . . . . 20

3.4 Longitudinal view of the CDF II tracking system. . . . . . . . . . . . . . . . 21

3.5 Layout of three COT SL2 supercells. . . . . . . . . . . . . . . . . . 23

3.6 Elevation view of one half of the $\mathrm{CDF}$ detector displaying the different components of the $\mathrm{CDF}$ calorimeter. . . . . . . . . . . . . . . . . . . . . . . 24

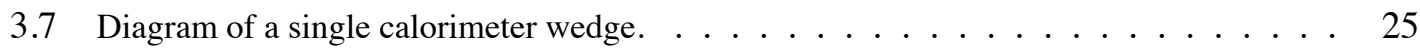

3.8 The EMTiming system layout. . . . . . . . . . . . . . . . . . . . . 27

3.9 General diagram of CDF Run II trigger and data acquisition systems. . . . . . . . . . . 29

3.10 Diagram of Level 1 and Level 2 trigger systems and the data flow between them. . . . . 30

3.11 Event Builder and L3 filtering. Data from the front end crates pass through ATM switches to the converter nodes. Here, the events are assembled and passed to the processor nodes. The accepted events are passed to output nodes which send them to the Consumer Server and Data Logging systems $(\mathrm{CS} / \mathrm{DL}) \ldots \ldots \ldots \ldots$. . . . . . . . . . . . 31

3.12 Schematic view of the luminosity monitor inside a quadrant of CDF. It is located at $|\theta|<3^{\circ} .32$

4.1 The invariant mass $M_{e e}$ distribution from $Z \rightarrow e^{+} e^{-}$data for the bin $90<E\left(e^{+}\right)+E\left(e^{-}\right)<110 \mathrm{GeV}$. The curve indicates the result of the fit to double Gaussian and a second-order polynomial background. . . . . . . . . . . . . . . . . 43

4.2 The energy scale correction from Z's $v s$ data period. Every data period corresponds to 25 months of data taking. The luminosities in each data period range from about $100 \mathrm{pb}^{-1}$

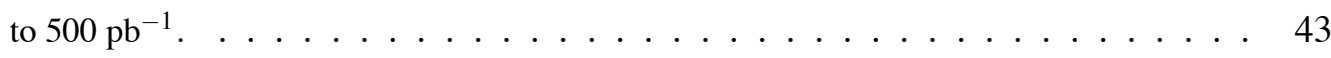

4.3 Efficiency of the PHOTON_25_ISO trigger as a function of the photon transverse momen-

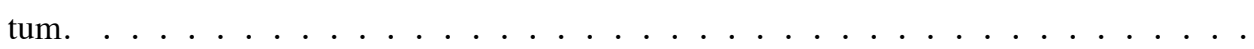

4.4 The efficiency of the level $2 \mathrm{HAD} / \mathrm{EM}<0.125$ requirement as a function of reconstructed photon $p_{T}$. The data are collected with the SUPER_PHOTON70 trigger. . . . . . . . . 45 
4.5 Efficiency of the SUPER_PHOTON70 trigger as a function of the $p_{T}$ of the photon. For $p_{T}^{\gamma}<120 \mathrm{GeV}$ the efficiency is measured with electrons in $Z \rightarrow e e$ decays. For higher $p_{T}$ the efficiency is measured relative to JET_100 trigger samples. . . . . . . . . . . . 46

4.6 MET $/ E_{T}^{\gamma}$ distribution for the inclusive photon sample (in red) and a Monte Carlo inclusive photon sample (in blue) for $80<p_{T}^{\gamma}<90 \mathrm{GeV} / \mathrm{c}$. The vertical green line indicates the separation between $M E T / E_{T}^{\gamma}<0.8$ and $M E T / E_{T}^{\gamma}>0.8 \ldots \ldots \ldots \ldots$

4.7 EMTiming distribution for beam halo and cosmic photons. The flat region is dominated by cosmic rays while the peak in the negative region is dominated by beam halos. There are turn-ons at $\sim-20 \mathrm{~ns}$ and fall-offs at $\sim 110 \mathrm{~ns}$ because the electronics gate opens about $20 \mathrm{~ns}$ before the collision and lasts for about $110 \mathrm{~ns}$ after it. . . . . . . . . .

4.8 Calorimeter isolation distribution for data, photon Monte Carlo and dijet Monte Carlo for $50<p_{T}^{\gamma}<60 \mathrm{GeV} / \mathrm{c}$. The distributions are normalized to their area, and the photon and dijet shapes are weighted according to the predicted signal fraction for this $p_{T}$ bin. . . .

$4.9 f_{\text {shift }}\left(p_{T}\right)=0.542 \cdot \tanh \left(-0.292+0.011 p_{T}-9.4 \cdot 10^{-5} p_{T}^{2}+5.1 \cdot 10^{-7} p_{T}^{3}\right)$ function that aligns the data and template peaks. . . . . . . . . . . . . .

$4.10 f_{o f f s e t}\left(p_{T}\right)=0.09-0.002 p_{T}$ function that centers the template at 0 before being reweighted. 54

$4.11 f_{\text {weight }}\left(p_{T}\right)=1.383-0.945 \cdot \tanh \left(8.32 \cdot 10^{-3} p_{T}\right)$ function that weights the peak width in the Monte Carlo template. . . . . . . . . . . . . . . . . . . . . . . 54

4.12 Signal fractions as a function of $p_{T}$ of the photon. The error bars associated to the points correspond to the statistical uncertainty in the fit, which comes from both the data and the Monte Carlo templates. The yellow band indicates the systematic uncertainty, described in detail in Chapter $5 . \ldots \ldots \ldots \ldots \ldots$

4.13 Fits to the isolation distribution in bins of $p_{T}$ for $30<p_{T}<44 \mathrm{GeV}$. Left column shows the fit results in linear scale. Logarithmic scales are displayed in right column. The signal template is from inclusive photon Monte Carlo, and the background template from dijet Monte Carlo with brem photons removed. . . . . . . . . . . . . . . .

4.14 Fits to the isolation distribution in bins of $p_{T}$ for $44<p_{T}<70 \mathrm{GeV}$. Left column shows the fit results in linear scale. Logarithmic scales are displayed in right column. The signal template is from inclusive photon Monte Carlo, and the background template from dijet Monte Carlo with brem photons removed. . . . . . . . . . . . . . . . . . 
4.15 Fits to the isolation distribution in bins of $p_{T}$ for $70<p_{T}<110 \mathrm{GeV}$. Left column shows the fit results in linear scale. Logarithmic scales are displayed in right column. The signal template is from inclusive photon Monte Carlo, and the background template from dijet Monte Carlo with brem photons removed. . . . . . . . . . . . . . . . .

4.16 Fits to the isolation distribution in bins of $p_{T}$ for $110<p_{T}<170 \mathrm{GeV}$. Left column shows the fit results in linear scale. Logarithmic scales are displayed in right column. The signal template is from inclusive photon Monte Carlo, and the background template from dijet Monte Carlo with brem photons removed. . . . . . . . . . . . . . . . . . .

4.17 Fits to the isolation distribution in bins of $p_{T}$ for $170<p_{T}<300 \mathrm{GeV}$. Left column shows the fit results in linear scale. Logarithmic scales are displayed in right column. The signal template is from inclusive photon Monte Carlo, and the background template from dijet Monte Carlo with brem photons removed. . . . . . . . . . . . . . . . . .

4.18 Fits to the isolation distribution in bins of $p_{T}$ for $300<p_{T}<400 \mathrm{GeV}$. Left column shows the fit results in linear scale. Logarithmic scales are displayed in right column. The signal template is from inclusive photon Monte Carlo, and the background template from dijet Monte Carlo with brem photons removed. . . . . . . . . . . . . . . . .

4.19 Unfolding factors as a function of the $p_{T}$ of the photon. The yellow band includes the systematic uncertainties due to the photon ID (acceptance + CES $\chi^{2}$ ) and the uncertainties due to the isolation and the energy scales (see next Chapter for details)

5.1 Summary of the signal fractions for all the different methods used to estimate the systematics together with the nominal result as a function of the photon $p_{T}$. The systematic uncertainty is indicated by the yellow band, and it is chosen to cover all the points. . . .

5.2 Fits of the $Z$ template for the two first bins $\left(30<p_{T}<34\right.$ and $\left.34<p_{T}<39 \mathrm{GeV} / \mathrm{c}\right)$. The $Z$ templates are fitted to the photon and dijet templates to estimate the residual background under the $Z$ mass peak. . . . . . . . . . . . . . . . . . . . . . .

5.3 The CES $\chi^{2}<20$ cut efficiency predicted by the photon Monte Carlo and with electrons from $Z$ 's in data as a function of the $p_{T}$ of the photon. . . . . . . . . . . . . 
5.4 The systematic uncertainty due to the CES $\chi^{2}$ efficiency as a function of the photon $p_{T}$. The red points are the efficiencies as measured by electrons from $Z$ decays, the green dots are the efficiencies predicted by the photon Monte Carlo, and the blue dots are the efficiencies measured with the inclusive photon sample. The systematic uncertainty covers the difference between the different estimations and is indicated by the yellow band.

5.5 Ratio of the energy scale in data and Monte Carlo $Z$ samples as a function of the sum of the energies of the two electrons from the $Z$ decay.

5.6 Comparison of the PHOTON_25_ISO trigger efficiencies obtained using electrons from Z's and electrons from $\mathrm{W}$ 's as a function of the photon $p_{T} \ldots \ldots \ldots \ldots$

5.7 Total systematic uncertainty and its relative contributions as a function of the $p_{T}$ of the photon. The systematic uncertainties are asymmetric, and its different contributions are detailed in Tables 5.2 and $5.3 \ldots \ldots \ldots \ldots \ldots$. . . . . . . . . . . . 76

6.1 The cross section as predicted by JETPHOX as a function of the isolation transverse energy in a cone $R=0.4$ around the photon for different bins of photon transverse momentum.

6.2 Ratio between the parton level cross section including the non-pQCD contributions and without including these contributions as predicted by PYTHIA Tune A samples. The correction to the JETPHOX theoretical predictions is given by the mean of the effects estimated with the Tune A set and with the Tune DW set. . . . . . . . . . . . . . . 82

6.3 Ratio between the parton level cross section including the non-pQCD contributions and without including these contributions as predicted by PYTHIA Tune DW samples. The correction to the JETPHOX theoretical predictions is given by the mean of the effects estimated with the Tune A set and with the Tune DW set. . . . . . . . . . . . . 83

6.4 Measured inclusive isolated photon cross section as a function of the photon $p_{T}$ compared to NLO pQCD predictions corrected for non-pQCD contributions. The yellow band includes the total systematic uncertainty on the measurement, except for the $6 \%$ luminosity uncertainty. 
6.5 Ratio DATA/THEORY as a function of the $p_{T}$ of the photon. The dot-dashed blue lines indicate the PDF uncertainty, and the dashed red line indicates the variation of the cross section when the renormalization, factorization and fragmentation scales are varied from $\mu=p_{T}$ to $\mu=p_{T} / 2$ and $\mu=2 P_{T}$. The theoretical prediction is corrected for the non-pQCD contributions. ....................... 86

6.6 Measured inclusive isolated photon cross section as a function of the photon $p_{T}$ compared to NLO pQCD predictions (top). The yellow band includes the total systematic uncertainty on the measurement except for the $6 \%$ luminosity uncertainty. The ratio DATA/THEORY as a function of the $p_{T}$ of the photon is presented below. The dot-dashed blue lines indicate the PDF uncertainty, and the dashed red lines indicate the variation of the cross section when the renormalization, factorization and fragmentation scales are varied from $\mu=p_{T}$ to $\mu=p_{T} / 2$ and $\mu=2 P_{T}$. The theoretical prediction is corrected for the non-pQCD contributions by $C_{U E}=0.913 \pm 0.004$ (stat) \pm 0.03 (sys), as shown in the bottom plot. . . . . . . . . . . . . . . . . . .

6.7 Ratio of data to theory predictions with CTEQ6.1M PDFs as a function of the $p_{T}$ of the photon (black dots) compared to the ratio of the predictions with CTEQ6.1M PDF to the predictions obtained with MRST04 PDF parametrization, indicated by the green line. The uncertainties due to the CTEQ6.1M PDF are indicated by the dot-dashed line, while the scale dependence is given by the dashed red line. The systematic uncertainties in the measurement are indicated by the yellow band. . . . . . . . . . . . . . .

A.1 Fits to the isolation distribution in bins of $p_{T}$ for $30<p_{T}<44 \mathrm{GeV}$. Left column shows the fit results in linear scale. Logarithmic scales are displayed in right column. The signal template is from $\mathrm{Z}$ data. The background template is from jet Monte Carlo with brem photons removed. . . . . . . . . . . . . . . . .

A.2 Fits to the isolation distribution in bins of $p_{T}$ for $44<p_{T}<70 \mathrm{GeV}$. Left column shows the fit results in linear scale. Logarithmic scales are displayed in right column. The signal template is from $\mathrm{Z}$ data. The background template is from jet Monte Carlo with brem photons removed. . . . . . . . . . . . . . . . . . . . 
B.1 Fits to the isolation distribution in bins of $p_{T}$ for $30<p_{T}<44 \mathrm{GeV}$. Left column shows the fit results in linear scale. Logarithmic scales are displayed in right column. The signal template is from photon Monte Carlo, the background template is from corrected

B.2 Fits to the isolation distribution in bins of $p_{T}$ for $44<p_{T}<70 \mathrm{GeV}$. Left column shows the fit results in linear scale. Logarithmic scales are displayed in right column. The signal template is from photon Monte Carlo, the background template is from corrected jet Monte Carlo with brem photons removed. . . . . . . . . . . . . . . . . .

B.3 Fits to the isolation distribution in bins of $p_{T}$ for $70<p_{T}<110 \mathrm{GeV}$. Left column shows the fit results in linear scale. Logarithmic scales are displayed in right column. The signal template is from photon Monte Carlo, the background template is from corrected jet Monte Carlo with brem photons removed. . . . . . . . . . . . . . . . . . . 100

B.4 Fits to the isolation distribution in bins of $p_{T}$ for $110<p_{T}<170 \mathrm{GeV}$. Left column shows the fit results in linear scale. Logarithmic scales are displayed in right column. The signal template is from photon Monte Carlo, the background template is from corrected jet Monte Carlo with brem photons removed. . . . . . . . . . . . . . . . 101

B.5 Fits to the isolation distribution in bins of $p_{T}$ for $170<p_{T}<300 \mathrm{GeV}$. Left column shows the fit results in linear scale. Logarithmic scales are displayed in right column. The signal template is from photon Monte Carlo, the background template is from corrected jet Monte Carlo with brem photons removed. . . . . . . . . . . . . . . . . . . . 102

B.6 Fits to the isolation distribution in bins of $p_{T}$ for $300<p_{T}<400 \mathrm{GeV}$. Left column shows the fit results in linear scale. Logarithmic scales are displayed in right column. The signal template is from photon Monte Carlo, the background template is from corrected jet Monte Carlo with brem photons removed.

C.1 Fit result for the bin $110<p_{T}<130 \mathrm{GeV}$ using non-corrected photon Monte Carlo templates. The peak in the photon Monte Carlo is wider and shifted to higher isolation values than the data. . . . . . . . . . . . . . . . . . . . . . 106

C.2 Data and Monte Carlo raw isolation in the region 1 for $150<p_{T}<170 \mathrm{GeV} / \mathrm{c}$. In this region the pile-up and the leakage contributions to the isolation energy are zero or negligible. 
C.3 Data and Monte Carlo raw isolation in the region 2 for $150<p_{T}<170 \mathrm{GeV} / \mathrm{c}$. In this region the pile-up together with the underlying event are the major contributors to the energy in the isolation cone. . . . . . . . . . . . . . . . . . . 109

C.4 Data and Monte Carlo corrected isolation in the region 2 for $150<p_{T}<170 \mathrm{GeV} / \mathrm{c}$. After the leakage correction (minor here) and the pile-up correction the discrepancies are not significantly worse than at raw level. . . . . . . . . . . . . . . . . . . 109

C.5 Data and Monte Carlo raw isolation in the region 3 for $150<p_{T}<170 \mathrm{GeV} / \mathrm{c}$. In this region the leakage contribution dominates. . . . . . . . . . . . . . . . . 110

C.6 Data and Monte Carlo leakage isolation in the region 3 for $150<p_{T}<170 \mathrm{GeV} / \mathrm{c}$. After the leakage corrections, differences are larger. . . . . . . . . . . . . . . . 110

C.7 Data and Monte Carlo corrected isolation for $150<p_{T}<170 \mathrm{GeV} / \mathrm{c}$. Red curve is Monte Carlo selected with $\mid$ cesx $\mid<15 \mathrm{~cm}$. Green curve is Monte Carlo with our nominal cuts and no $a d$ hoc correction. . . . . . . . . . . . . . . . . . . . . . . . . . 111

C.8 Energy in the random cone for data (black) and Monte Carlo (red) for $130<p_{T}<150 \mathrm{GeV} / \mathrm{c} .112$

D.1 Data/PYTHIA vs the $p_{T}$ of the photon. . . . . . . . . . . . . . . . . . 113

D.2 Comparison of the $p_{T}$ of the photon in data (after the background is subtracted) and Monte Carlo before the reweighting function is applied to the Monte Carlo. The distributions are normalized to their area . . . . . . . . . . . . . . . . . . . . . . . 114

D.3 Comparison of the $p_{T}$ of the photon in data (after the background is subtracted) and Monte Carlo after the reweighting function is applied to the Monte Carlo. The distributions are normalized to their area. . . . . . . . . . . . . . . . . . . 115 


\section{List of Tables}

2.1 Summary of the direct photon experiments whose results are presented in Fig. 2.6. The name of the experiment is given in the first column, in the second column the name of the corresponding accelerator is indicated. The next columns detail the particles in the initial state, the center of mass energy, and the year when the experiment started. . . . . . . . 12

3.1 Summary of the characteristics of the CDF Run II calorimeters. . . . . . . . . . . 26

4.1 The different level requirements of the PHOTON_25_ISO trigger, applied for $30<p_{T}^{\gamma}<90 \mathrm{GeV} / \mathrm{c}$. Energies are in $\mathrm{GeV} . \ldots \ldots \ldots$

4.2 The different requirements for all the levels of the SUPER_PHOTON70_EM and the SUPER_PHOTON70_JET triggers, applied for $p_{T}^{\gamma}>90 \mathrm{GeV} / \mathrm{c}$. The SUPER_PHOTON70 trigger path is an OR of these two. Energies are in GeV. . . . . . . . . . . . . . . . . 36

4.3 The applied photon cuts. Energies are in $\mathrm{GeV} \ldots \ldots \ldots \ldots$. . . . . . . . 41

4.4 The applied photon-like electron cuts. Energies are in GeV. . . . . . . . . . . . . . 42

4.5 Trigger efficiencies $\left(\varepsilon_{T}\right)$, signal fractions $(\mathcal{F})$ together with the systematic uncertainties, and unfolding factors $(U)$ with the associated statistical uncertainties as a function of the photon transverse momentum. The trigger efficiencies correspond to the PHOTON_25_ISO trigger for $p_{T}<90 \mathrm{GeV} / \mathrm{c}$, while the SUPER_PHOTON70 efficiencies are those for $p_{T}>90 \mathrm{GeV} / \mathrm{c}$. In the trigger, the statistical uncertainties are smaller than $1 \%$ for all the measured range, while the systematic uncertainties are small and are neglected. All the systematic uncertainties are described in detail in Chapter $5 . \ldots 63$ 
5.1 2-bin method results compared to the fit result when the templates are divided at isolation of $1 \mathrm{GeV}$. . . . . . . . . . . . . . . . . . . . . .

5.2 Positive systematic uncertainties (in $\%$ ) for every $p_{T}$ bin. $\mathcal{F}$ is for signal fraction (blue line in Fig. 5.7), photon ID includes both the uncertainty due to the acceptance and the CES $\chi^{2}$ uncertainty (brown line in Fig. 5.7), and $e-$ scale and iso-scale are the energy and the isolation scales uncertainties (red and green lines respectively in Fig. 5.7). . . . .

5.3 Negative systematic uncertainties (in $\%$ ) for every $p_{T}$ bin. $\mathcal{F}$ is for signal fraction (blue line in Fig. 5.7), photon ID includes both the uncertainty due to the acceptance and the CES $\chi^{2}$ uncertainty (brown line in Fig. 5.7), and $e-$ scale and iso-scale are the energy and the isolation scales uncertainties (red and green lines respectively in Fig. 5.7). . . . .

6.1 The cross section result and the corresponding theoretical prediction. The latter does not include the correction due to the non pQCD effects, $C_{U E}=0.913 \pm 0.004$ (stat) \pm 0.03 (sys). The statistical and systematic uncertainties in the measurement are also presented, both in $\%$. The statistical errors come from the data, and also take into account the small effect of the limited statistics in the Monte Carlo samples employed for the unfolding factors (see Chapter 4), while the systematic uncertainties are asymmetric and are explained in detail in Chapter 5. The luminosity uncertainty, of 6\%, is not included in the Table. . . . 


\section{Chapter 1}

\section{Introduction}

In this thesis we present the measurement of the inclusive isolated prompt photon cross section with a total integrated luminosity of $2.5 \mathrm{fb}^{-1}$ of data collected with the CDF Run II detector at the Fermilab Tevatron Collider. The prompt photon cross section is a classic measurement to test perturbative QCD (pQCD) [1] with potential to provide information on the parton distribution function (PDF), and sensitive to the presence of new physics at large photon transverse momentum. Prompt photons also constitute an irreducible background for important searches such as $\mathrm{H} \rightarrow \gamma \gamma$, or SUSY and extra-dimensions with energetic photons in the final state.

The Tevatron at Fermilab (Batavia, U.S.A.) is currently the hadron collider that operates at the highest energies in the world. It collides protons and antiprotons with a center-of-mass energy of $1.96 \mathrm{TeV}$. The CDF and the D0 experiments are located in two of its four interaction regions. In Run I at the Tevatron, the direct photon production cross section was measured by both CDF and DO [2], and first results in Run II have been presented by the DO Collaboration based on $380 \mathrm{pb}^{-1}$ [3]. Both Run I and Run II results show agreement with the theoretical predictions except for the low $p_{T}^{\gamma}$ region, where the observed and predicted shapes are different. Prompt photon production has been also extensively measured at fixed-target experiments [4] in lower $p_{T}^{\gamma}$ ranges, showing excess of data compared to the theory, particularly at high $x_{T}$.

From an experimental point of view, the study of the direct photon production has several advantages compared to QCD studies using jets. Electromagnetic calorimeters have better energy resolution than hadronic calorimeters, and the systematic uncertainty on the photon absolute energy scale is smaller. Furthermore, the determination of the photon kinematics does not require the use of jet algorithms. However, the measurements using photons require a good understand- 
ing of the background, mainly dominated by light mesons $\left(\pi^{0}\right.$ and $\left.\eta\right)$ which decay into two very collinear photons. Since these photons are produced within a jet, they tend to be non-isolated in most of the cases, and can be suppressed by requiring the photon candidates to be isolated in the calorimeter. In the case the hard scattered parton hadronizes leaving most of its energy to the meson, the photon produced in the decay will not be surrounded by large energy depositions. To further reduce this remaining isolated background, we present a new technique based on the isolation distribution in the calorimeter. The measured cross section is compared to next-to-leading order (NLO) pQCD calculations, which have been corrected for non-perturbative contributions.

This thesis is organized as follows: we start with a brief review of QCD theory and the formalism to calculate cross sections in Chapter 2, where we also introduce the physics of prompt photon production and summarize the current status of the prompt photon phenomenology. Chapter 3 contains a description of the Tevatron and the CDF detector. The experimental measurement is described in Chapter 4, where we provide details on the different datasets used in the measurement, the trigger, and the event selection requirements. Most of this Chapter is devoted to the explanation of the background subtraction method and the determination of the photon signal fraction. The systematic uncertainties on the measurement are evaluated in Chapter 5, while Chapter 6 discusses the final results and the comparison to the theoretical predictions. Finally, the conclusions are presented in Chapter 7. 


\section{Chapter 2}

\section{Theory}

In this chapter we first present the basis of the formalism to calculate cross sections in QCD, and then we focus on the particular case of the photon production, discussing the physics, the phenomenology and the theoretical calculations related to this process. Finally, we provide a short summary of the Monte Carlo simulation tools used in the experimental measurement.

\subsection{Hadron Scattering Formalism}

Quantum Chromodynamics (QCD) is the theory that describes the strong interactions in the Standard Model. The strength of the strong interactions is set by the strong coupling constant $\alpha_{s}$, and its dependence with the energy scale is given by the $\beta\left(\alpha_{s}\right)$ function. The evolution of the running coupling constant with $Q^{2}$ is given by the renormalization group equation:

$$
Q^{2} \frac{d \alpha_{s}}{d Q^{2}}=\beta\left(\alpha_{s}\left(Q^{2}\right)\right)
$$

where the $\beta$ function is calculated perturbatively. At leading order, the renormalization group equation is solved by

$$
\alpha_{S}\left(Q^{2}\right)=\frac{1}{b_{0} \ln \left(Q^{2} / \Lambda_{Q C D}^{2}\right)}
$$

where $b_{0}$ is a LO coefficient which depends on the scale $Q^{2}$ and $\Lambda_{Q C D}$, and indicates the order of magnitude of the scale at which $\alpha_{s}\left(Q^{2}\right)$ becomes non-perturbative, typically chosen to be of the order of $200 \mathrm{MeV}$. Fig. 2.1 shows the predicted QCD running of the coupling constant compared 
to several measurements in different processes. The running of the strong coupling constant is such that the strength of the interaction decreases with the increasing of the energy scale. Therefore, at short distances the partons behave essentially as free particles, while at large distances the strength of the coupling asymptotically diverges, bounding quarks and gluons into colourless hadrons. These two phenomena are known as asymptotic freedom and colour confinement [6].

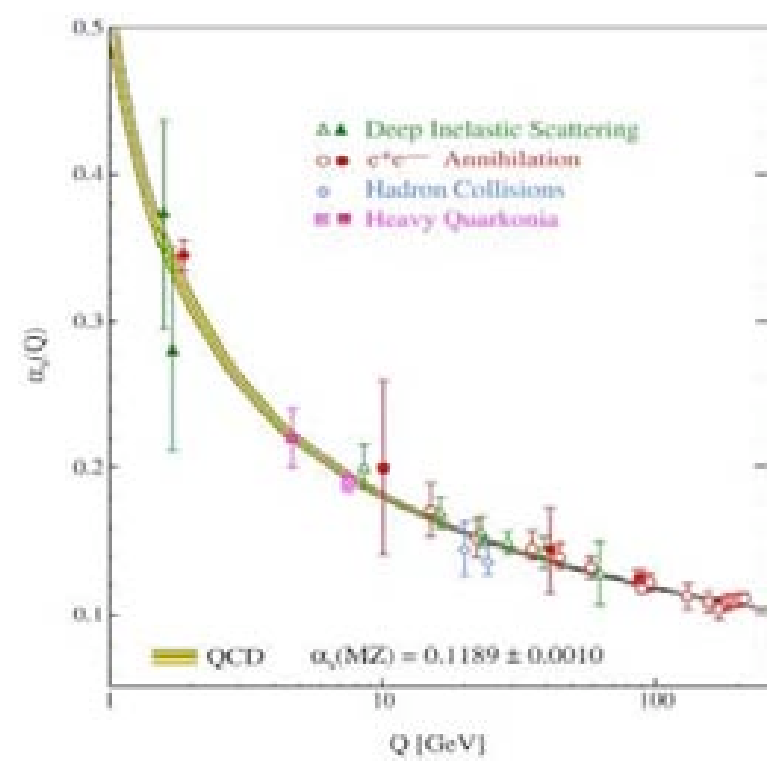

Figure 2.1: Summary of measurements of the strong coupling $\alpha_{s}\left(Q^{2}\right)$ as a function of the respective energy scale $Q$. The curves are the QCD predictions for the combined world average value of $\alpha\left(M_{Z}^{2}\right)$. Figure taken from [7].

QCD provides the formalism to calculate the cross sections for interactions involving hadrons in the initial or the final state. The factorization theorem holds that the cross section of any QCD process can be written as the convolution of three basic building blocks (PDF, partonparton cross section, fragmentation) that separate the high-energy (perturbative) processes from the low-energy (non-perturbative) physics effects. The cross section of any interaction between two hadrons $H_{1}+H_{2} \rightarrow H_{3}+X$ can be expressed as (Fig. 2.2):

$$
\sigma\left(P_{1}, P_{2}\right)=\sum_{i, j, k} \int d x_{1} d x_{2} d z_{3} f_{i / 1}\left(x_{1}, \mu_{F}^{2}\right) f_{j / 2}\left(x_{2}, \mu_{F}^{2}\right) D_{k / 3}\left(z_{k}, \mu_{f}^{2}\right) \hat{\sigma_{i j k}}\left(p_{1}, p_{2}, p_{3}, \alpha_{s}\left(\mu_{F}^{2}\right), Q^{2} / \mu_{F}^{2}\right)
$$

Here $P_{1,2}$ are the momenta of the incoming hadrons and $p_{1,2}=x_{1,2} P_{1,2}$ the momenta of the partons which participate in the hard scattering process. The sum runs over all parton types, whose proba- 
bility density to be found within the hadron $H_{1}$ with momentum fraction $x_{1}$ at a given factorization scale $\mu_{F}$ is given by the parton distribution function (PDF) $f_{i / 1}\left(x_{1}, \mu_{F}\right)$. The factorization scale is an arbitrary parameter introduced to handle singularities in the calculation that cannot be treated perturbatively. These singularities are caused by soft physics effects such as collinear radiation, and are absorbed into the parton distribution functions at a given scale $\mu_{F}$, usually chosen to be of the order of the hard scale $Q^{2}$. The PDFs cannot be determined by perturbative QCD calculations but its functional form is parametrized from experimental data at a fixed scale $Q_{0}^{2}$. Fortunately, they are defined in a way that they are universal and their evolution with the factorization scale is predicted by the DGLAP (Dokshitzer, Gribov, Lipatov, Altarelli, Parisi) equations [8], so the PDFs measured at one scale can be used to predict the results of experiments at other scales. There are different sets of PDF parametrizations. In this thesis, the Next-to-Leading Order (NLO) calculations are done using the CTEQ6.1M PDFs set, whose predictions for the $u, \bar{u}, d$ quarks and the gluon at scale $Q^{2}=1000 \mathrm{GeV}^{2}$ are shown in Fig. 2.3. Other parametrizations are, for example, Alekin2004 [10] and MRST2004 [11].

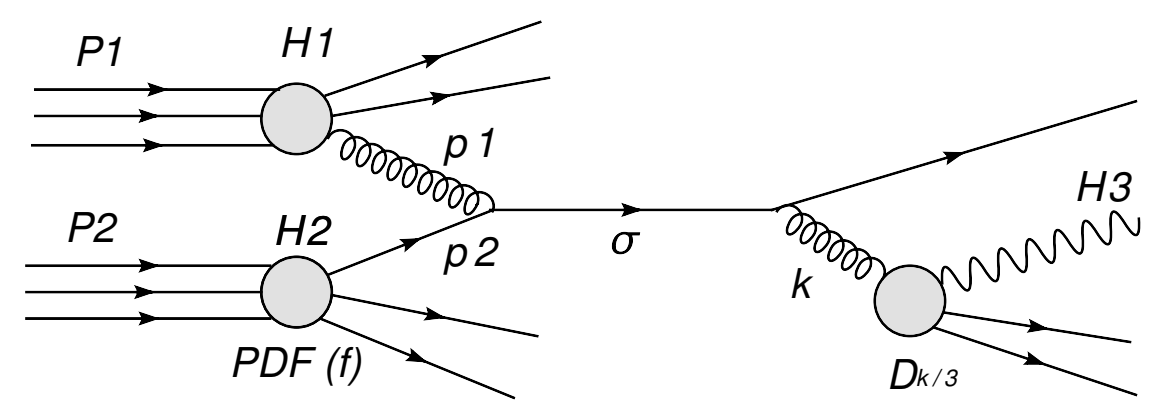

Figure 2.2: Diagram of the interaction between two hadrons. The process is described as the convolution of the Parton Density Functions (PDFs) of the partons inside the incoming hadrons. The hard scattering is described by the partonic cross section, $\sigma$, which can be calculated perturbatively. The outgoing partons can fragment into other particles. This process is taken into account by the fragmentation functions (Ds).

$D_{k / 3}\left(z_{k}, \mu_{f}^{2}\right)$ is the fragmentation function and gives the probability that the produced parton produces final state particle $H_{3}$ with momentum fraction $z_{3}$ during the fragmentation process at some fragmentation scale $\mu_{f}$. The fragmentation scale $\mu_{f}$ is introduced the same way as the factorization scale and under similar prescription. It intends to absorb singularities due to finalstate collinear radiation. Like the PDFs, the fragmentation functions are not calculable, but we can calculate their dependence with the scale. The fragmentation functions only appear in the calculation when the final state particle is the result of the fragmentation process of the parton 


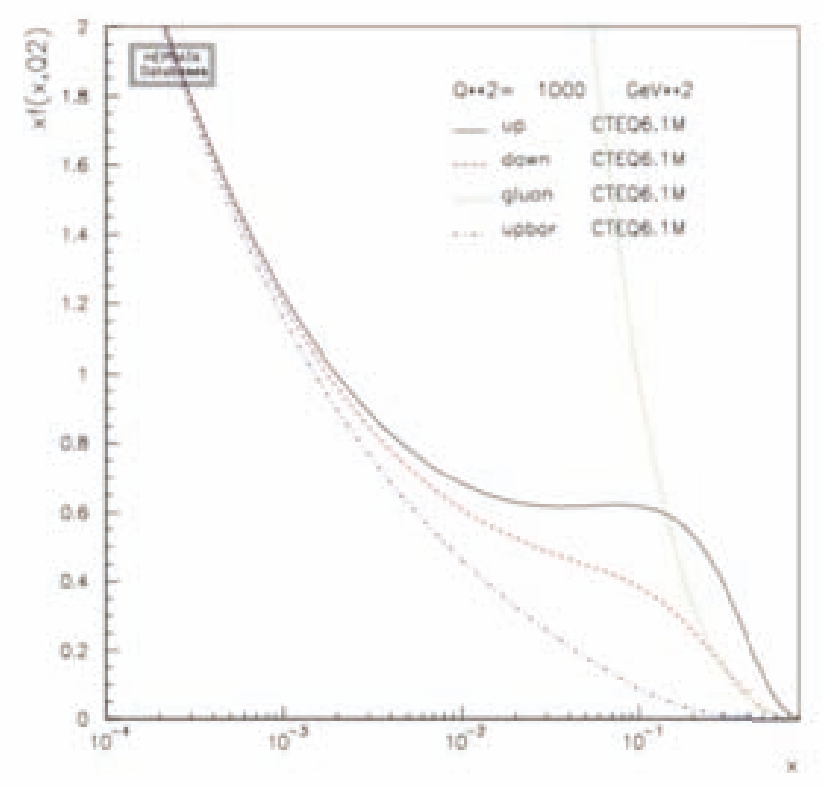

Figure 2.3: Example of proton PDFs for $Q^{2}=1000 \mathrm{GeV}^{2}$ as given by CTEQ6.1M [9].

produced in the hard scattering process. In our case, prompt photons can be produced directly in the hard interaction collision (direct photons) or as a result of the fragmentation of a quark or a gluon into a photon (fragmentation photons). In the first case no fragmentation function is needed for describing the process but in the second case the contribution to the cross section will depend on $D_{\gamma / q}$ and $D_{\gamma / g}$. The determination of these functions will be discussed with more detail in Section 2.2.

$\sigma_{i j}$ is the parton cross section interaction, calculated at a given order of pQCD and at a renormalization scale $\mu_{R}$. The renormalization scale is introduced to absorb the ultraviolet singularities that appear at higher than LO orders in the perturbative calculations. It is usually chosen to be of the same value of $\mu_{F}$ and $\mu_{f}$. There is no reason for these three scales to be exactly the same, but they should not be chosen to be very different from each other because this would introduce an unphysical hierarchy into the calculation. Since they are totally arbitrary, any physical observable must be independent from their particular choice. If a calculation were carried out to all orders in perturbation theory, there would not be any dependence left in the final result. However, most calculations are available at a fixed order, and therefore residual dependence on the scales is left. The variation of the cross section with the scale reflects the size of the uncalculated terms in the 
perturbative expansion.

$$
\mu^{2} \frac{d}{d \mu^{2}} \sum_{i=0}^{N} \alpha_{s}^{i} \sigma_{i}=O\left(\alpha_{s}^{N+1}\right)
$$

In this thesis we have chosen $\mu_{R}=\mu_{F}=\mu_{f}=p_{T}^{\gamma}$ for the calculation of the nominal pQCD predictions. In order to evaluate the effect of higher order terms in the predictions we re-calculated the cross section by fixing these scales to be $2 p_{T}^{\gamma}$ and $p_{T}^{\gamma} / 2$. The choice of the PDFs and fragmentation functions employed in the theory calculation also leaves some residual dependence in the predictions that must be taken into account when comparing to the experimental data. The uncertainties in the theory due to the particular choice of the parametrization of the PDFs are evaluated using the Hessian method [12] (see Chapter 6 for more details on the calculation).

\subsection{Prompt photon production}

The production of inclusive prompt photon provides a stringent test of $\mathrm{pQCD}$ predictions over several orders of magnitude [9], and its measurement offers some unique advantages over jets. First, the presence of the QED vertex at tree level makes the theory calculations more reliable. The process also gives access to lower $p_{T}$ 's than jets, where the underlying event contamination reduces the sensitivity of the QCD measurements. Photons do not hadronize, so there is no need for arbitrary jet definitions, and the photon energies can be measured with electromagnetic rather than hadronic calorimeters, resulting in improved energy resolution. One of the main motivations for prompt photon measurements is their potential to constrain the gluon distribution of the proton. This is due to the gluon appearance in the initial state of the tree level Compton diagrams (Fig. 2.4) which dominate the photon cross section at the Tevatron at low-to-moderate $p_{T}$. The process is also sensitive to the presence of new physics at very high $p_{T}$, and constitutes an irreducible background for important searches, such as $H \rightarrow \gamma \gamma$, or SUSY and extra-dimensions with energetic photons in the final state.

Prompt photons can be produced directly from the hard scattering process or as a result of the collinear fragmentation of a parton that is itself produced with a large transverse momentum. The tree level contributions to direct process are shown in the four first diagrams of Fig. 2.4. The two upper diagrams describe the $q \bar{q}$ annihilation process and the other two are the QCD analog of Compton scattering in QED. Fig. 2.4 also shows two examples of diagrams that may contribute to the fragmentation or bremsstrahlung process of a parton into a photon. These processes are described in general as $2 \rightarrow 2$ hard scattering convoluted with the fragmentation functions, $D_{\gamma / q}$ 
and $D_{\gamma / g}$, though for large scales the calculation can be done perturbatively, as shown in the diagram on the left.
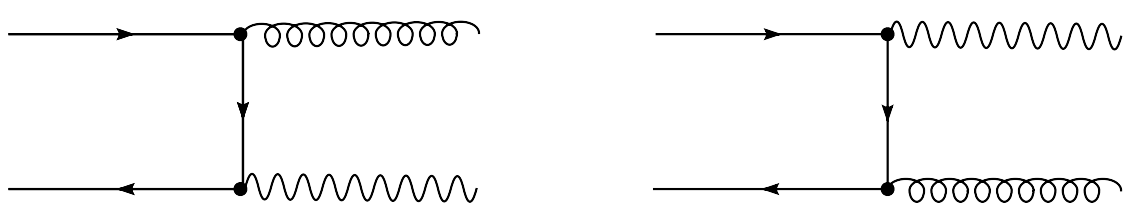

Annihilation
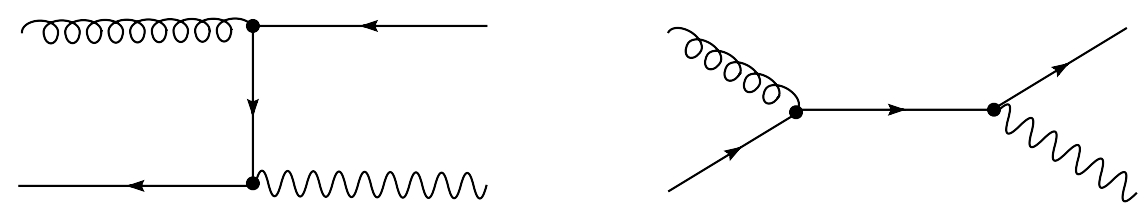

Compton $Q C D$
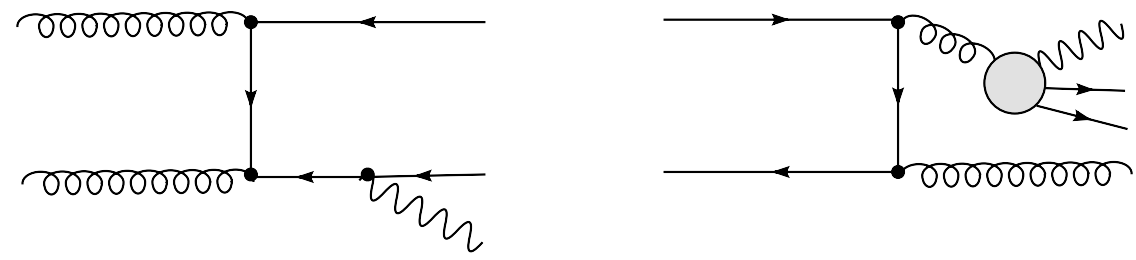

Fragmentation

Figure 2.4: Prompt photon production diagrams. The two upper diagrams show the $q \bar{q}$ annihilation process $q \bar{q} \rightarrow g \gamma$. The second row of diagrams correspond to the Compton process $q(\bar{q}) g \rightarrow q(\bar{q}) \gamma$. The two diagrams in the bottom show two examples of fragmentation contributions. The left one is the pointlike fragmentation of a quark into a photon, which can be calculated perturbatively for asymptotically large scales. The right one is the non-perturbative fragmentation of a gluon producing a photon.

At LO, the differential cross section as a function of the transverse momentum $p_{T}$ and the pseudorapidity ${ }^{1} \eta$ of the photon can be written as the sum of the direct and the fragmentation contributions:

$$
\frac{d \sigma\left(\mu_{R}, \mu_{F}, \mu_{f}\right)}{d p_{T} d \eta}=\frac{d \sigma_{D}\left(\mu_{R}, \mu_{F}, \mu_{f}\right)}{d p_{T} d \eta}+\frac{d \sigma_{f}\left(\mu_{R}, \mu_{F}, \mu_{f}\right)}{d p_{T} d \eta}
$$

The distinction between these two mechanisms has no physical meaning beyond LO [13]. The separation between them in the theoretical calculations is arbitrary, only their sum has physical

\footnotetext{
${ }^{1}$ The pseudorapidity is defined as a function of the polar angle of the photon $\theta$ as $\eta=-\ln (\tan (\theta / 2))$.
} 
meaning and can be compared to the experimental cross section. The fragmentation part can be expressed synthetically as:

$$
\frac{d \sigma_{f}\left(\mu_{R}, \mu_{F}, \mu_{f}\right)}{d p_{T} d \eta}=\sum_{k=q, \bar{q}, g} \frac{d \sigma_{k}^{f}\left(\mu_{R}, \mu_{F}, \mu_{f}\right)}{d p_{T} d \eta} \otimes D_{\gamma / k}\left(\mu_{f}\right)
$$

$\sigma_{k}^{f}$ describes the production of a parton $k$ in the hard collision and $D_{\gamma / k}$ accounts for the fragmentation function of the parton $k$ into the photon. When the fragmentation occurs at large scales ${ }^{2}$, the process can be described by perturbation theory (as shown in the left diagram of Fig. 2.4). However, the contribution from soft QCD processes (such as the illustrated in the right diagram of Fig. 2.4 or the soft component of the left diagram of the same figure) cannot be treated perturbatively. In this case $D_{\gamma / k}$ is determined experimentally from $e^{+} e^{-} \rightarrow q q \gamma$ data at LEP and other electron machines [14] using the Vector Meson Dominance Model (VDM). In this model, one assumes the quarks and gluons first fragment into vector mesons which then turn into photons. The photon is described by a linear combination of vector mesons such as the $\rho(770), \omega(782)$ and $\phi(1020)$ and the input of $D_{\gamma / k}$ at a fixed scale is determined from $D_{\gamma / \rho}, D_{\gamma / \omega}$ and $D_{\gamma / \phi}$ at this same scale. These meson-to-photon fragmentation functions can be determined using data from ALEPH [15] and HRS [16]. The resulting fragmentation functions were compared to ALEPH data in [14], providing a direct check of the VDM approach. In this thesis, to calculate the theoretical prediction of the cross section, we employ BFG set II [57] parametrization of fragmentation functions.

\subsubsection{Isolated prompt photon calculations}

From the experimental point of view, the challenge of the prompt photon cross section measurement is the identification of the photon signal. This task is complicated by the decays of light mesons such $\pi^{0}$ and $\eta$ in two photons. Since they interact hadronically, light mesons are produced in great quantities in hadron machines, and all photon measurements must have highly efficient methods to remove these decays from their datasets. Fixed target experiments generally measure the cross section for photon $p_{T}$ less than $10 \mathrm{GeV}$. At these energies, the $\pi^{0} \rightarrow \gamma \gamma$ and the $\eta \rightarrow \gamma \gamma$ decays produce two photons which can be resolved by the calorimeter, and therefore fixed target experiments can provide inclusive measurements of the prompt photon cross section. However, measurements at collider experiments are performed at higher energies, making very difficult the distinction between the decays and single photon showers in an event-by-event basis. To deal

\footnotetext{
${ }^{2} \mu_{f}^{2} \sim p_{T}^{2} \sim 25 \mathrm{GeV}^{2}$ in fixed-target direct photon experiments [14]
} 
with such background, hadron colliders usually measure only the isolated part of the photon cross section. The isolation transverse energy of the photon $\left(E_{T}^{i s o}\right)$ is measured in the calorimeter as the transverse energy deposited within a cone of radius $R$ centered in the photon cluster minus the energy of the photon itself:

$$
E_{T}^{i s o}=E_{T}(R)-E_{T}^{\gamma}
$$

Photons from meson decays are produced within a jet, with large energy depositions around them, so they come as non-isolated photons in most of the cases. The isolaton requirement (typically $E_{T}^{i s o}<1-2 \mathrm{GeV}$ ) highly suppresses these contributions. However, in the case the hard scattered parton hadronizes leaving most of its energy to a light meson, the two photons from the decay will look as an isolated photon. Therefore, even after the isolation requirement is imposed, photon precision measurements at hadron colliders need statistical background subtraction techniques to further remove the remaining isolated photons from the datasets.

To face up this experimental reality, new theoretical calculations of the photon isolated cross section were developed. Nowadays, full NLO calculations are implemented at the partonic level in flexible Monte Carlo programs. Programs of this type account for experimental cuts in an easy way, match naturally the binning of experimental data and, by histograming of the partonic configurations generated, allow for a straightforward study of correlations. In particular, these NLO calculations easily allow the implementation of the isolation requirement at the parton level in the inclusive calculations.

The prove of the applicability of the factorization theorem to the isolated prompt photon cross section in hadron interactions was first available in [13]. The authors of this study demonstrated that the calculations of the isolated case were infrared and collinear safe and that both the PDFs and the fragmentation functions were independent of the process and of the isolation requirements. Therefore, the partonic cross sections in the isolated case are still calculable with perturbative QCD.

In this thesis the measured cross section is compared to NLO perturbative QCD prediction from the JETPHOX Monte Carlo program [22]. JETPHOX is a general purpose cross section integrator, designed to calculate both single photon inclusive and photon+jet inclusive cross sections and related correlations. The isolated cross section is obtained from the inclusive one by subtracting the part that does not satisfy the isolation constraints:

$$
d \sigma^{i s o}=d \sigma^{i n c}-d \sigma^{s u b}\left(R, E_{T}^{i s o}\right)
$$

Fig. 2.5 shows the comparison of the JETPHOX predictions at LO and NLO for the isolated 
prompt photon production. The photons are required to be central $(|\eta|<1)$ in the calorimeter, have $E_{T}^{\gamma}>30 \mathrm{GeV}$ and $E_{T}^{i s o}<2 \mathrm{GeV}$. The NLO calculations include the full description of the fragmentation component, which is not totally accounted for at LO accuracy. The effect of the fragmentation in the cross section appears to be important at low energies, where the NLO result doubles the LO prediction. The effect tends to decrease as the photon energy increases.

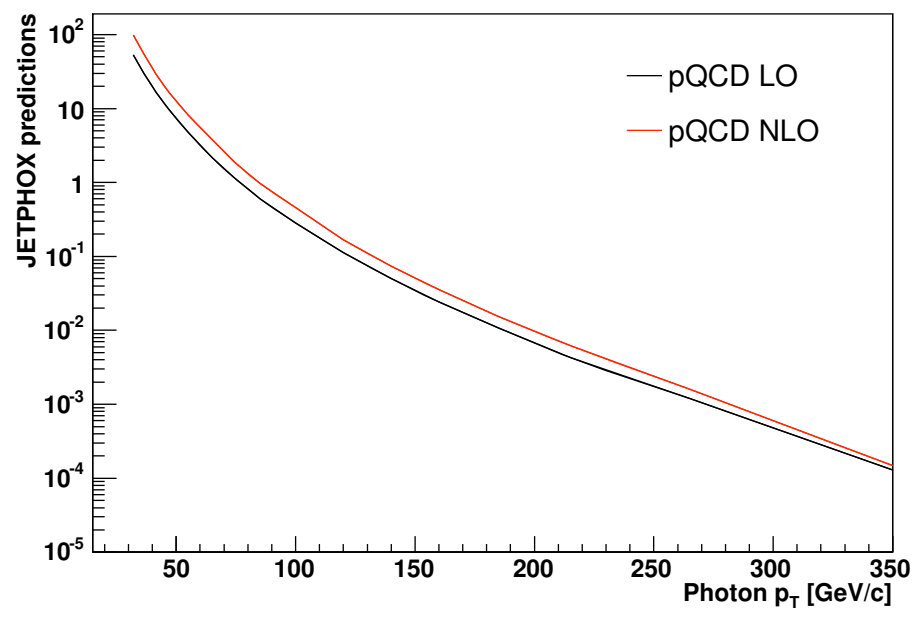

Figure 2.5: Comparison of the JETPHOX perturbative QCD predictions at LO and NLO for central $(|\eta|<1)$ photons with $E_{T}^{\gamma}>30 \mathrm{GeV}$ and $E_{T}^{i s o}<2 \mathrm{GeV}$.

\subsubsection{Previous measurements}

Many years of intense experimental and theoretical efforts had contributed to the understanding of the inclusive prompt photon production in hadron collisions. A large variety of experiments have measured the photon cross section at both fixed target and collider energies (see Table 2.1 for a summary), but no consensus has been reached concerning the phenomenology of these processes. Various experimental results tend to fall above the theoretical predictions. The initial partons may possess some 'intrinsic $k_{T}$ ' due to the fact that they are bound within a nucleon of finite size, though this effect is believed too small (of the order of 0.3-0.5 GeV/c) to explain the observed enhancement. This situation motivated the introduction of an extra non-perturbative parameter to account for the effects of multiple soft gluon emissions associated to the hard partonic scattering [17], so the colliding partons would possess a small amount of transverse momentum $\left(k_{T}\right)$ 
due to the recoil of the system, and the smearing of the initial-state center-of-mass energy would increase the cross section because of its steep slope as a function of $p_{T}$.

However, the $k_{T}$ hypothesis remains somewhat controversial. Authors of [18] affirm there is no need for an additional $k_{T}$ to force the agreement between QCD predictions and experiments, with the possible exception of one data set from the E706 experiment. The normalization of these data is of the order of 5 times higher than in the other fixed target experiments (see Fig. 2.6). The introduction of the $k_{T}$ parameter brings the E706 data closer to the theory predictions, but the agreement found with other datasets when no $k_{T}$ parameter is considered is no longer observed. They conclude this data is inconsistent with other experiments and that no serious discrepancy exists between data and theory. Overall, there is no definite theoretical method to parametrize the $k_{T}$ smearing effects and different groups obtain rather different shifts on the differential cross sections, especially at low $p_{T}[19]$.

\begin{tabular}{ccccc}
\hline \hline Experiment & Accelerator & Initial state & $\sqrt{s}$ & year \\
\hline R806 & ISR & $p p$ & $63 \mathrm{GeV}$ & 1982 \\
WA70 & SPS & $p p$ & $23 \mathrm{GeV}$ & 1988 \\
UA1 & $S p \bar{p} S$ & $p \bar{p}$ & $630 \mathrm{GeV}$ & 1988 \\
R110 & ISR & $p p$ & $63 \mathrm{GeV}$ & 1989 \\
R807 & ISR & $p p$ & $63 \mathrm{GeV}$ & 1990 \\
UA2 & $S p \bar{p} S$ & $p \bar{p}$ & $630 \mathrm{GeV}$ & 1991 \\
UA6 & $S p \bar{p} S$ & $p p$ & $24.3 \mathrm{GeV}$ & 1998 \\
UA6 & $S p \bar{p} S$ & $p \bar{p}$ & $24.3 \mathrm{GeV}$ & 1998 \\
E706 & Tevatron fixed target & $p B e$ & $31.6 \mathrm{GeV}$ & 1998 \\
E706 & Tevatron fixed target & $p B e$ & $38.8 \mathrm{GeV}$ & 1998 \\
DO & Tevatron collider & $p \bar{p}$ & $630 \mathrm{GeV}$ & 2000 \\
DO & Tevatron collider & $p \bar{p}$ & $1800 \mathrm{GeV}$ & 2001 \\
CDF & Tevatron collider & $p \bar{p}$ & $630 \mathrm{GeV}$ & 2001 \\
CDF & Tevatron collider & $p \bar{p}$ & $1800 \mathrm{GeV}$ & 2001 \\
\hline \hline
\end{tabular}

Table 2.1: Summary of the direct photon experiments whose results are presented in Fig. 2.6. The name of the experiment is given in the first column, in the second column the name of the corresponding accelerator is indicated. The next columns detail the particles in the initial state, the center of mass energy, and the year when the experiment started.

This situation has prevented the use of the inclusive photon data for the PDF fits since year 


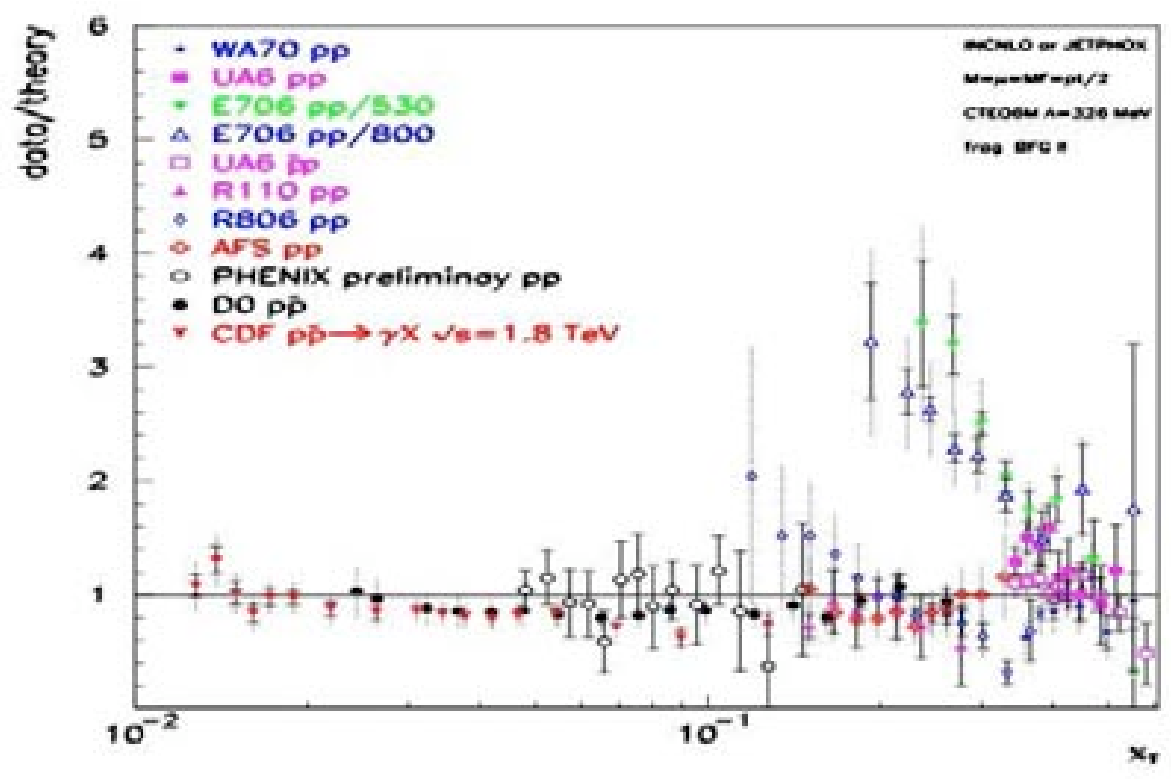

Figure 2.6: Ratio data to theory for collider and fixed target data with scales $\mu=p_{T} / 2$. Measurements for fixed target data at low energies are inclusive, while DO and CDF data come from isolated measurements. Figure taken from [21].

1998. Currently, the gluon PDF is determined from global fits to jet data from the Tevatron, to HERA data and data from fixed-target experiments, but the uncertainty in the gluon distribution at high $x$ is still quite large. Therefore, it is important to incorporate further constraints on the gluon, and the introduction of the photon data has a high potential to improve the knowledge of the gluon PDF. However, prior to including photon data in the PDF fits, the controversy about the $k_{T}$ enhancement needs to be solved. This particularly affects the $p_{T}$ region of the fixed-target experiments, while for collider data this is less an issue because the $k_{T}$ affects only for $p_{T}<30$ $\mathrm{GeV} / \mathrm{c}$ or so [20]. Therefore, collider data might be used for the PDF global fits in the region between $30<p_{T}<200 \mathrm{GeV} / \mathrm{c}$ and $0.03<x_{T}<0.2$, where the Compton scattering dominates, if reasonable agreement between data and theory is found. Because of the dominance of the $q \bar{q}$ scattering subprocess in the Tevatron photon cross sections at high $p_{T}$, a large change in the gluon distribution is required to generate relatively small change in the cross section, increasing the uncertainty in the gluon PDF at high $x$. The results of this thesis will help to improve the understanding of prompt photon production and hopefully will contribute to constrain the gluon PDF for $0.03<x_{T}<0.2$. 


\subsection{Monte Carlo Event Generators}

The Monte Carlo simulation programs constitute an essential tool for experimental high energy physics. The applications of Monte Carlo simulations are extensive. For example, they can be used to study effects of the detector calibration, efficiencies of identification selections, or to generate the expected background events in the data sample.

Starting from an initial hadron-hadron collision, the Monte Carlo programs include models to simulate the final particles measured in the detector. The simulation chain starts with the generation of the LO $2 \rightarrow 2$ hard scattering between the two initial partons and follows up with the Parton Shower approximation, where the partons produced in the hard scattering process emit other partons, producing a cascade. The successive parton radiation is governed by the splitting functions for gluon radiation, gluon splitting and quark pair production. The shower can evolve backward or forward in time, and therefore these programs allow the possibility to include the effect of initial-state radiation processes taking into account the different PDFs involved in the hard collision. In the final state, the point at which the cascade stops is set by the $\Lambda_{Q C D}$ parameter, usually of the order of $1 \mathrm{GeV}$.

At this point the partons are recombined together to form hadrons. This process does not imply large momentum transfers and cannot be treated perturbatively. Its simulation is based on the hypothesis of the local parton-hadron duality, which states that hadrons are produced by partons that are close in phase-space [23]. Therefore, the flow of momentum and quantum numbers at the hadron level tends to follow the one established at the parton level, and the transition from a partonic to a hadronic jet does not wash out the original parton kinematics and flavor information. The longitudinal and transverse momentum distributions are substantially unchanged and the final state hadrons are collimated into a small angular region in the direction of the original parton, forming jets. The process of the hadron formation is only described by models based on parameters that have to be determined experimentally. The two most used models are the cluster model [6] and the string model [24].

The Monte Carlo programs are designed to provide a realistic simulation of the interactions of the particles with the detector material. They fully simulate the detector effects and include models to incorporate in the simulations the effect caused by non-perturbative processes. In hadronhadron colliders, the presence of hadron remnants that do not participate in the hard interaction leads to soft underlying event activity that contribute to the final state. The proper treatment of the underlying event involves taking into account their colour connections with the hard interaction. 
Their contributions to the final state are modeled as "minimum bias" processes with $\hat{p_{T}} \geq p_{T}^{\hat{m i n}}$ and flat in rapidity, where $p_{T}^{\hat{m} i n}$ has a typical value around 1.5-2.5 GeV/c. In CDF, this contribution has been tuned to correctly reproduce the experimental results using observables that are specially sensitive to the underlying event modeling, as for example, jet shapes and event profiles.

\subsubsection{PYTHIA Monte Carlo}

PYTHIA is the Monte Carlo event generator used in this thesis for the Monte Carlo samples. It uses LO Matrix Element calculations to generate hard interactions between partons [25] and it is optimized for $2 \rightarrow 1$ and $2 \rightarrow 2$ processes.

An event in PYTHIA is initiated by two particles coming toward each other, each particle characterized by a PDF. One parton from each particle starts off a sequence of branchings which build up an initial-state shower. The hard process occurs between one parton of each of these showers, producing two outgoing particles which may branch building up final-state showers. After the parton shower, the parton fragmentation and hadronization mechanisms gather the partons together into singlet colour states. The hadronization in PYTHIA is performed using string fragmentation. Most of the hadrons produced during the fragmentation are unstable particles and decay to final and stable particles which finally deposit their energy in the detector, simulated using a GEANT program [26].

In the case of direct photon production, the photon produced in the hard interaction is calculated directly at Matrix Element level in the $2 \rightarrow 2$ process. The contributions to this process are the $q \bar{q}$ annihilation and the QCD Compton, whose diagrams are detailed in Fig. 2.4. These photons are mostly isolated, similarly to the direct photons in JETPHOX. However, prompt photons can also be produced during the parton shower process if one of the final state partons radiates an early photon (see first fragmentation diagram of Fig. 2.4). Due to this mechanism, about $15 \%$ of the events may contain two high $p_{T}$ photons. Similarly to the fragmentation component in JETPHOX, the bremsstrahlung photons are accompanied by other radiated particles and therefore will usually appear non-isolated.

The description of the underlying event in PYTHIA is done through different models, the Tune A being the default set of parameters at CDF. PYTHIA Tune A has been tuned to reproduce specific measurements performed by the CDF experiment during the Run I of the Tevatron [29], and its settings mainly affect the initial state showers from the incoming hadrons, where the scale $Q^{2}$ is increased and the lower cut-off decreased to allow more radiation. The probability 
that the multiple parton interaction produces two gluons with colour connections to their nearest neighbours is also increased. An alternative parametrization to Tune A is the Tune DW [30], very similar to Tune $\mathrm{A}$, but that in addition describes accurately the distribution of the transverse momentum of the $Z / \gamma^{*}$ boson at very low values $p_{T}^{Z}<5 \mathrm{GeV} / \mathrm{c}$. 


\section{Chapter 3}

\section{The CDF Detector}

The data used in this analysis was collected by the Collider Detector at Fermilab (CDF) during the Tevatron Run II. This chapter is devoted to the description of the Tevatron accelerator chain and the main CDF detector subsystems, and concludes with a detailed explanation of the trigger and the offline photon reconstruction at CDF.

\subsection{The Tevatron}

The Tevatron is a proton antiproton superconducting collider located at Fermilab (Batavia, IL, U.S.A.). It collides 36 proton and 36 antiproton bunches at a center-of-mass energy of $\sqrt{s}=1.96 \mathrm{TeV}$ every $396 \mathrm{~ns}$, and the CDF and DO experiments are located at two of its four interaction points. A picture of the Fermilab's accelerator chain is shown in Fig. 3.1. The first stage of the acceleration process starts with the Cockcroft-Walton electrostatic accelerator, where hydrogen gas $\left(\mathrm{H}_{2}\right)$ is ionized to create negative ions $\left(H^{-}\right)$that are accelerated to $750 \mathrm{KeV}$. These ions are inserted in a linear accelerator (Linac), a 150 meter long chain of radio-frequency accelerator cavities, where they reach an energy of $400 \mathrm{MeV}$. Before entering the third stage, the ions pass through a carbon foil which strips the electrons, leaving only the protons, which are injected into the Booster, a 75 meter radius synchrotron. In the Booster the protons are split into bunches while accelerated to $8 \mathrm{GeV}$ before going into the Main Injector, another synchrotron with a circumference of $3 \mathrm{~km}$ where the proton bunches are merged together in higher density bunches which are accelerated to $150 \mathrm{GeV}$. Finally in the Tevatron, 36 proton bunches, separated by $396 \mathrm{~ns}$, are accelerated to $980 \mathrm{GeV}$. 


\section{FERMILAB'S ACCELERATOR CHAIN}

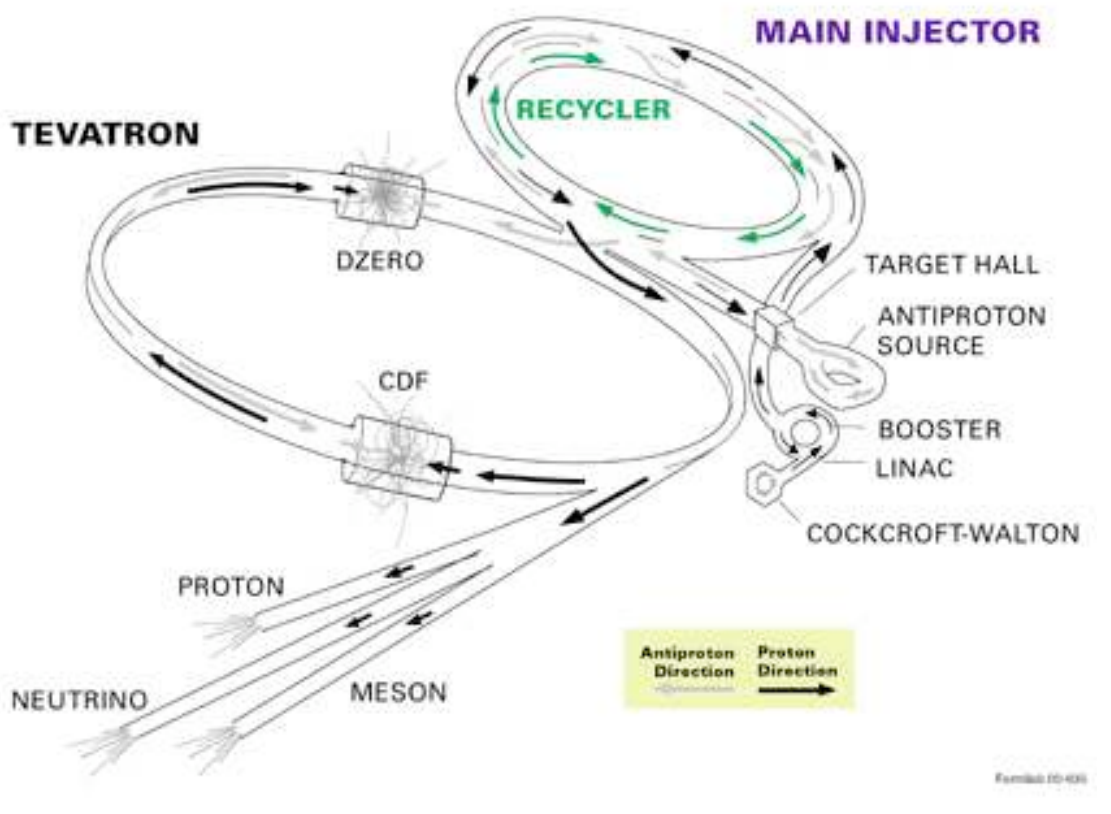

Figure 3.1: The accelerator complex at FNAL.

The production of antiprotons is significantly more complicated and its production rate is the major limiting factor in the available instantaneous luminosity at the Tevatron. First, $120 \mathrm{GeV}$ protons are extracted from the Main Injector to hit a nickel target, producing antiprotons among a variety of different particles, with an efficiency of $2 \cdot 10^{-5}$ antiproton/proton. The antiprotons are separated from the other produced particles and focused into a beam that is stored in the Accumulator by a system of pulsed magnets and lithium lens. When enough antiprotons are accumulated (about $10^{12}$ ), they are transferred back to the Main Injector where their energy is increased to $150 \mathrm{GeV}$. Finally, they enter to the Tevatron for the final acceleration to energies of $980 \mathrm{GeV}$.

The number of collisions per second is described by the instantaneous luminosity $\mathcal{L}$, which can be expressed as:

$$
\mathcal{L}=\frac{f N_{B} N_{p} N_{\bar{p}}}{2 \pi\left(\sigma_{p}^{2}+\sigma_{\bar{p}}^{2}\right)} F\left(\sigma_{l} / \beta^{*}\right)
$$

where $f$ is the revolution frequency in $\mathrm{Hz}, N_{B}$ is the number of bunches, $N_{p(\bar{p})}$ is the number of protons (antiprotons) per bunch, and $\sigma_{p(\bar{p})}$ is the protons (antiprotons) RMS beam size at the 
interaction point. This is multiplied by a form factor, $F$, that depends on the ratio of the bunch longitudinal RMS size, $\sigma_{l}$, and the beta function at the interaction point, $\beta^{*}$, a measure of the transverse beam width.

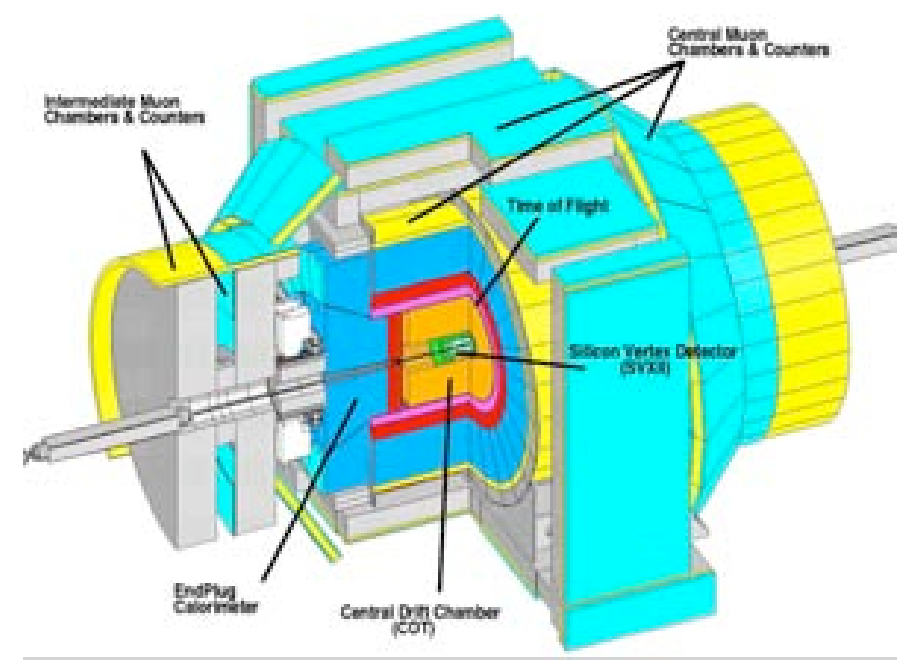

Figure 3.2: Isometric view of the CDF Run II detector with its main subsystems (innermost to outermost): the Silicon Vertex Detector (green), the Central Outer Tracker (orange), the superconducting solenoid (pink), the electromagnetic calorimeter (red), the hadronic calorimeter (blue) and the muon chamber (yellow and blue).

\subsection{The CDF Run II Detector}

The CDF Run II detector, shown schematically in Fig. 3.2, is a multipurpose experiment designed to study high energy $p \bar{p}$ collisions. CDF is a cylindrical-shaped detector with azimuthal and forward-backward symmetry and uses a cylindrical coordinate system $(z, \eta, \phi)$, with the origin set at the geometrical center of the detector (see Fig. 3.3). The $z$ axis lays along the proton beam direction, the $y$ axis points upward and the $x$ axis $(\phi=0)$ lays in the accelerator plane pointing away from the center of the ring.

Due to the fact that the protons and antiprotons are extended objects, the actual constituent partons will not be traveling at $980 \mathrm{GeV}$ but they will have different longitudinal velocities. Therefore, in hadron colliders, we must use variables that are invariant under longitudinal boosts. Instead of the energy or the momentum, only transverse quantities, such as the transverse energy 


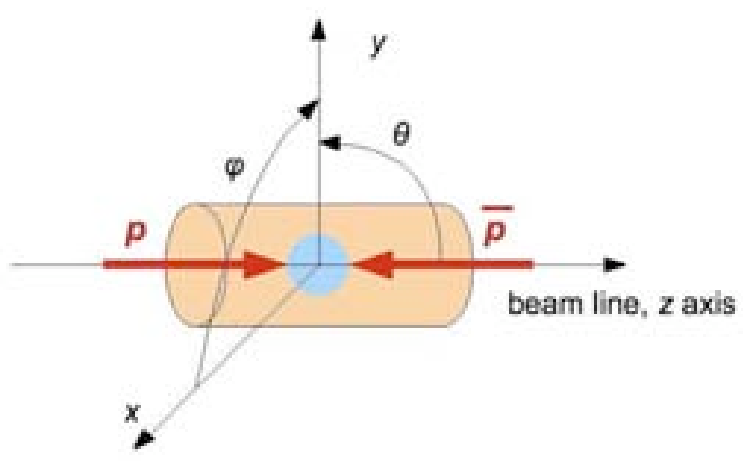

Figure 3.3: The CDF coordinate system.

$E_{T}=E \sin \theta$ or the transverse momentum $p_{T}=p \sin \theta$ are useful.

The rapidity, $y$, of a particle is given by

$$
y=\frac{1}{2} \ln \frac{E+p_{z}}{E-p_{z}}
$$

and it is invariant under a Lorentz boost transformation. The pseudorapidity, $\eta$, defined as

$$
\eta=-\ln (\tan (\theta / 2))
$$

equals the rapidity $y$ in the massless approximation $(p>>m)$ and it is extensively used because of its direct geometric interpretation.

The CDF detector is formed by different subdetectors each designed to measure specific particles in the event. In the next sections we describe the main subsystems of the CDF detector, following the path of a particle coming from the interaction point.

\subsubsection{Tracking Systems}

The tracking system of the CDF detector is the closest subsystem to the beam pipe and is formed by the silicon detector and the Central Outer Tracker (COT), which are surrounded by a superconducting solenoid magnet that provides a $1.4 \mathrm{~T}$ magnetic field parallel to the beam axis. The $\eta$ coverage of the system is shown in Fig. 3.4 together with other subdetectors. 


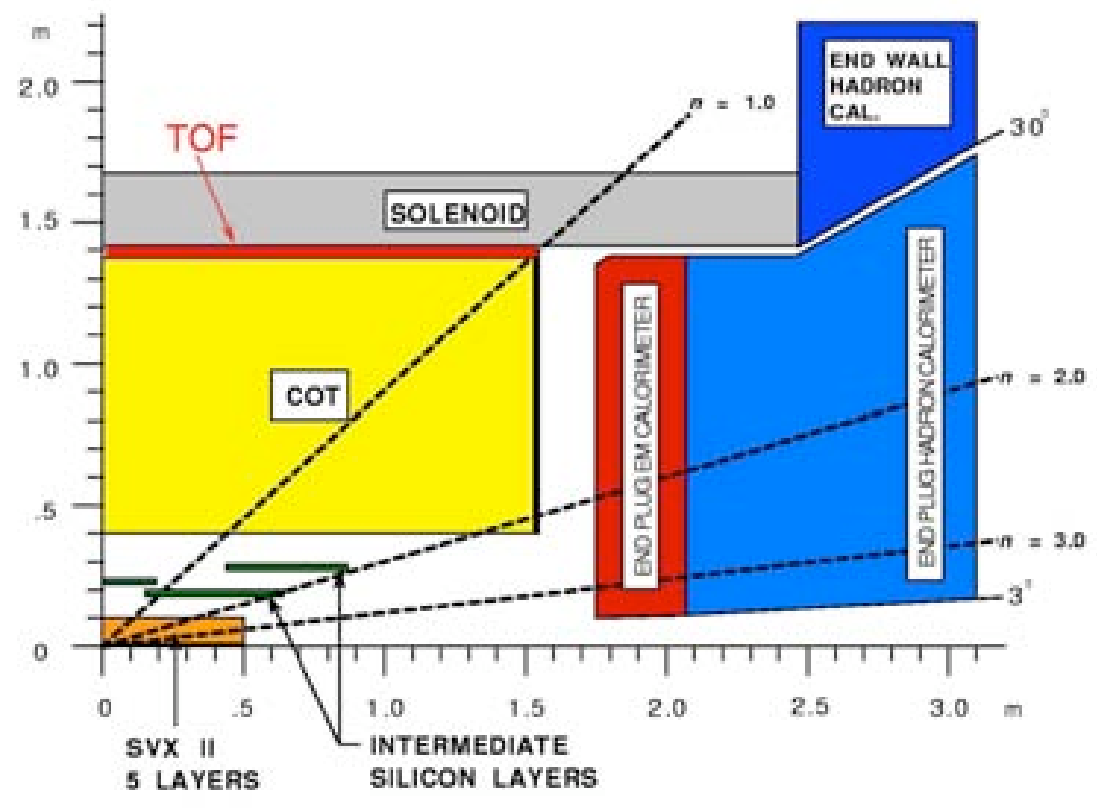

Figure 3.4: Longitudinal view of the CDF II tracking system.

\subsubsection{Silicon Detector}

The silicon detector [32] is a 8-layer silicon micro-strip tracker designed to provide high resolution in the measurement of the impact parameter and to increase the acceptance of the outer tracker in the forward regions of the detector. The system is composed by 3 subdetectors and covers the region $|\eta|<2.8$ (Fig. 3.4). The innermost layer (Layer00) goes from $r=1.35 \mathrm{~cm}$ to $2.4 \mathrm{~cm}$ and is mounted directly on the beam pipe. The 5 layers of the Silicon Vertex Detector (SVX II) expand from $r=2.4 \mathrm{~cm}$ to $10.6 \mathrm{~cm}$ to perform precision tracking measurement and triggering. The Intermediate Silicon Layers (ISL) are located between the SVX II and the main CDF tracking chamber (COT), and link the silicon measurements with those of the COT, extending the coverage of the detector in the forward region. The SVX II and the ISL layers are double-sided detectors, while the L00 is single-sided silicon. They achieve together about $10 \mu \mathrm{m}$ resolution in single hit measurements, $20 \mu \mathrm{m}$ on impact parameter for tracks of $p_{T}>3 \mathrm{GeV} / \mathrm{c}$, and $40 \mu \mathrm{m}$ in the determination of the intersection point of a track with the beam line, where the beam line itself has a size of $30 \mu \mathrm{m}$. 


\subsubsection{Central Outer Tracker}

The COT [33] is a cylindrical, open cell, multiwire drift chamber surrounding the silicon detector. The cylinder is $310 \mathrm{~cm}$ long and it radially expands from $r=40 \mathrm{~cm}$ to $137 \mathrm{~cm}$, providing full coverage of the region of $|\eta|<1$ and with a maximum acceptance of $|\eta|<2$ (see Fig. 3.4). It is divided into 8 "superlayers" (SL) which are in turn divided in the azimuthal direction into "supercells". The supercells have a maximum drift distance that is approximately the same for all the superlayers, so the number of supercells in a given SL scales with the radius. Fig. 3.5 shows the layout of a supercell. In the COT, "axial" and "stereo" superlayers alternate. Axial SL have the wires running parallel to the $z$ axis, while the wires in "stereo" SL are strung at a small angle $\left(2^{\circ}\right)$ with respect to the $z$ direction. The combination of axial and stereo layers provide information of $z$ and $r-\phi$ positions.

The COT is filled with an Argon-Ethane (50:50) gas mixture and Isopropyl alcohol. The Argon-Ethane mixture provides a constant electron drift velocity across the cells, while the Isopropyl is added to reduce aging effects on the wires. Since the COT is immersed in a $1.4 \mathrm{~T}$ magnetic field, the electrons drift at a Lorentz angle of $35^{\circ}$. Supercells are tilted by the same amount with respect to the radial direction to compensate for this effect. Single hit resolutions in the COT of $140 \mu \mathrm{m}$ translate into transverse momentum resolutions of $\sigma\left(p_{T}\right) / p_{T}=$ $(0.15 \%) \times p_{T}\left[(\mathrm{GeV})^{-1}\right]$. The resolution improves to $(0.07 \%) \times p_{T}\left[(\mathrm{GeV})^{-1}\right]$ if silicon tracking information is added. In addition to the measurement of charged particle momenta, the COT can be used to identify particles with $p_{T}<2 \mathrm{GeV} / \mathrm{c}$ based on $d E / d x$ measurements.

\subsubsection{Time of Flight}

The Time of Flight (TOF) detector lies between the COT and the solenoid (see Fig. 3.4) and is formed by 2163 meter long scintillating bars located at $\mathrm{r} \sim 140 \mathrm{~cm}$ with one photo-multiplier tube attached to both ends. Every bar covers $1.7^{\circ}$ in $\phi$ and $|\eta|<1$. The TOF detector is used to distinguish between low momentum pions, kaons and protons by using the time they take to travel from the primary vertex to the system with a resolution of $\sim 100 \mathrm{ps}$.

\subsubsection{Calorimeters}

The primary purpose of the calorimeters is to measure the energy of neutral and charged particles in the final state. An schematic view of the CDF calorimeters is shown in Fig. 3.6; they are 


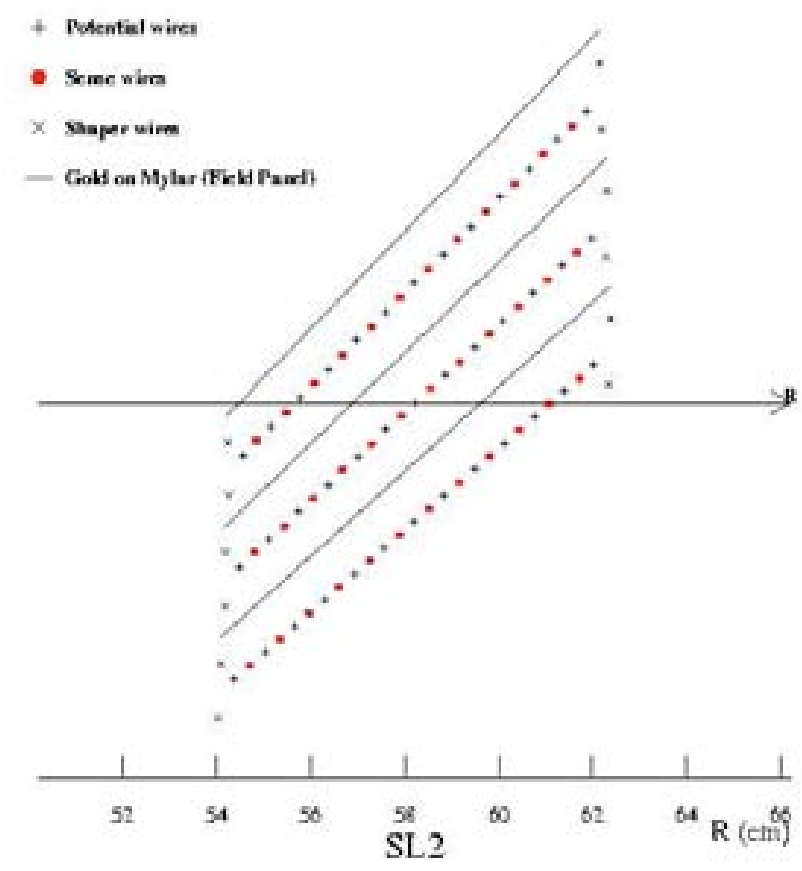

Figure 3.5: Layout of three COT SL2 supercells.

non compensated sampling calorimeters that use scintillating plastic as active material. Located after the solenoid coil, the CDF calorimeter system is formed by two calorimeters, the central, up to $\eta=1.1$, and the forward (or "plug") calorimeter, that extends to $|\eta|<3.64$. The central calorimeter is divided in two halves at $\eta=0$, leaving two gaps at $\eta=0$ and $\eta=1$. The central and the plug calorimeters are segmented into 1536 projective towers pointing to the center of the detector, distributed along in 48 wedges of $15^{\circ}$ in azimuth and 0.1 units in pseudorapidity. The light produced by the shower particles crossing a scintillating plate is collected by a wavelength shifting (WLS) fibers that transport it to photomultiplier tubes (PMT) located in the outermost part of the calorimeters. Every projective tower is read by one or two PMTs, depending on the calorimeter.

\subsubsection{Central Calorimeters}

The central calorimeter is subdivided into an inner electromagnetic detector, the central electromagnetic calorimeter (CEM) [35], and two outer hadronic detectors, called the central hadronic 


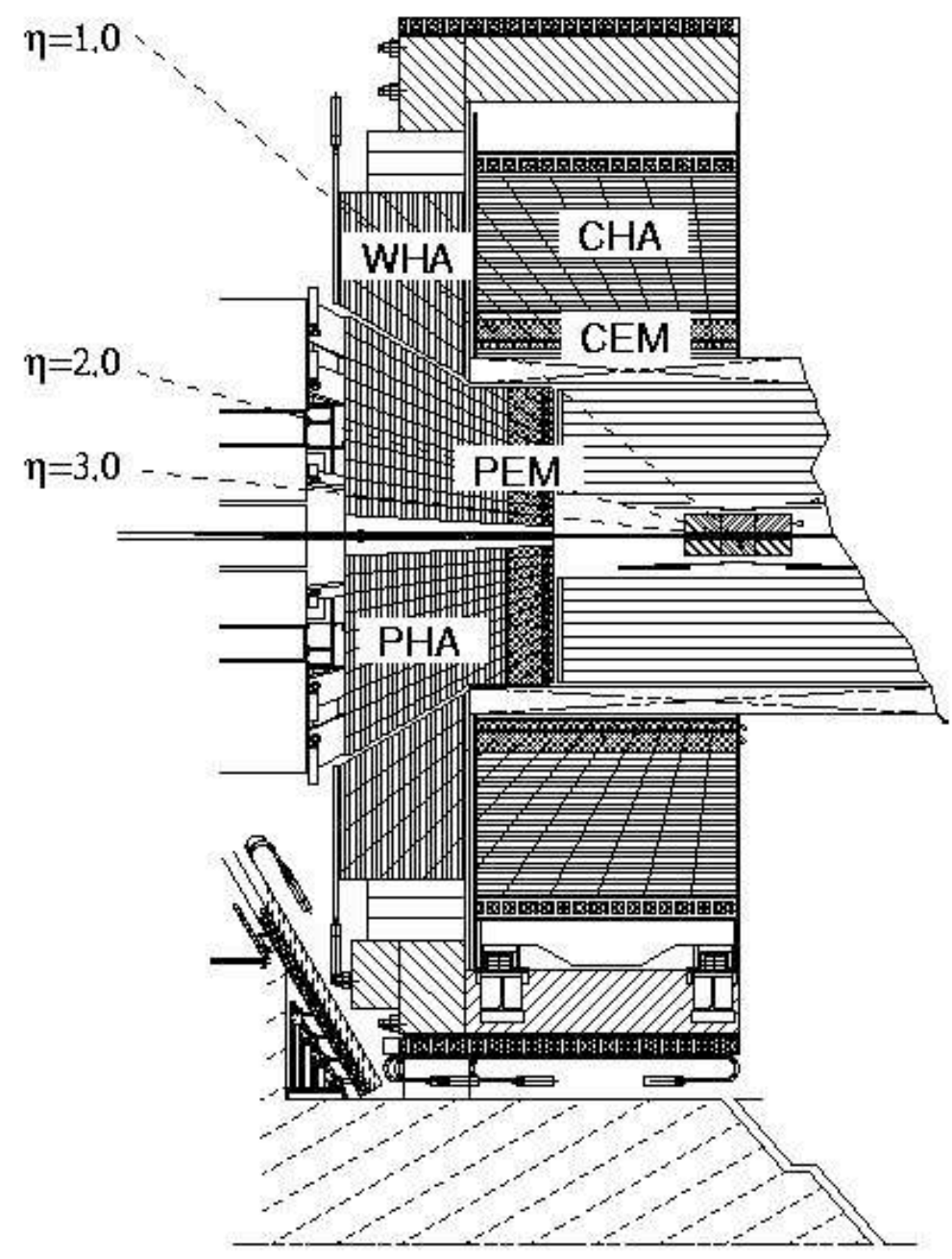

Figure 3.6: Elevation view of one half of the CDF detector displaying the different components of the $\mathrm{CDF}$ calorimeter.

and end-wall calorimeters (CHA, WHA) [36]. A cutaway cross-section view of a central calorimeter wedge is shown in Fig. 3.7. The CEM has a depth of 18 radiation lengths $\left(X_{0}\right)$ and is surrounded by the central hadronic calorimeter (CHA), whose coverage is extended by the WHA in the region $0.9<|\eta|<1.3$. Both the CHA and the WHA have a depth of 4.7 interaction lengths $\left(\lambda_{I}\right)$. Each tower in the central calorimeters is a set of plastic scintillator tiles interleaved with lead as a sampling material in the case of CEM, and with steel in the case of CHA and WHA. 
The central calorimeter towers are read by two WLS fibers and two PMTs, located at the low and high- $\phi$ side of the tower, which produce around 40 photoelectrons per GeV.

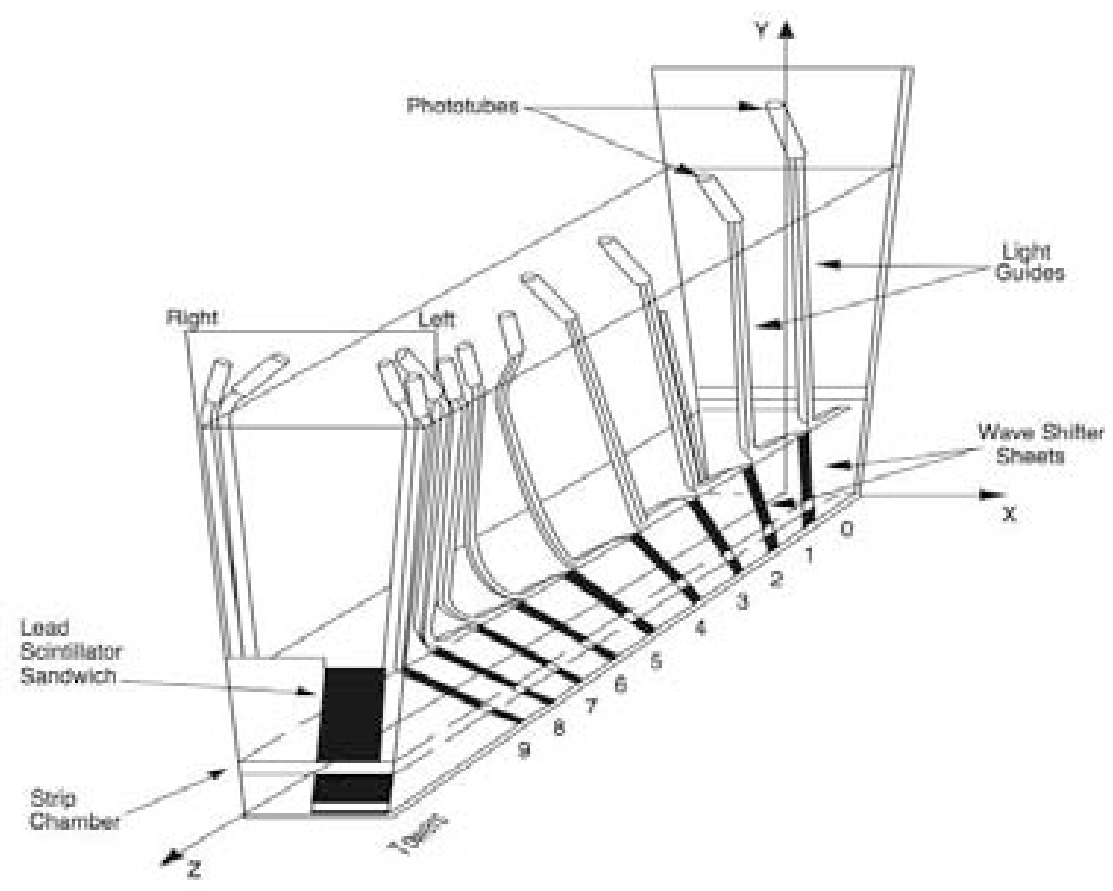

Figure 3.7: Diagram of a single calorimeter wedge.

The energy resolution of each segment of the calorimeter for a single particle was measured using a testbeam and can be parametrized as:

$$
\left(\frac{\sigma}{E_{T}}\right)^{2}=\left(\frac{a}{\sqrt{E_{T}}}\right)^{2}+b^{2}
$$

where the first term comes from sampling fluctuations and the photo-statistics of the PMTs, the second term comes from the intercalibration between the different towers due to the non-uniform response of the calorimeter, and $E_{T}$ is the transverse energy of the particle. For the CEM, the energy resolution (expressed in $\mathrm{GeV}$ ) of the high-energy photons and electrons is $\frac{\sigma}{E_{T}}=\frac{13.5 \%}{\sqrt{E_{T}}} \oplus$ $2 \%$. In CHA and WHA detectors the energy resolution was obtained using charged pions, giving $\frac{\sigma}{E_{T}}=\frac{50 \%}{\sqrt{E_{T}}} \oplus 3 \%$ for the CHA and $\frac{\sigma}{E_{T}}=\frac{75 \%}{\sqrt{E_{T}}} \oplus 4 \%$ for the WHA. 


\subsubsection{Forward Calorimeters}

The forward or "plug" calorimeters [37] are also divided in electromagnetic (PEM) and hadronic (PHA) parts, the PEM with $23 X_{0}$ and the PHA with $6.8 \lambda_{I}$. In the plug calorimeters, each tower is a set of plastic scintillator tiles interleaved with lead as a sampling material in the case of PEM, and with iron in the case of the PHA. The $\eta$ segmentation of the towers varies from 0.1 to 0.6 depending on the pseudo-rapidity region, while the segmentation in $\phi$ varies from $7.5^{\circ}$ in the region $1.1<|\eta|<2.1$ to $15^{\circ}$ in the region $2.1<|\eta|<3.6$. The light produced in response to the energy deposited is collected using WLS fibers and derived to the PMTs to produce around 300 photoelectrons per $\mathrm{GeV}$. The energy resolution as a function of the total energy (in $\mathrm{GeV}$ ) for the plugs was determined in the test beam to be $\frac{\sigma}{E}=\frac{16 \%}{\sqrt{E}} \otimes 1 \%$ for PEM and $\frac{\sigma}{E}=\frac{80 \%}{\sqrt{E}} \otimes 5 \%$ for PHA. The characteristics of the CDF calorimeters are summarized in Table 3.1.

\begin{tabular}{ccccc}
\hline \hline Calorimeter & Coverage & Segmentation $(\eta \times \phi)$ & Thickness & Resolution $[E$ in GeV] \\
\hline CEM & $|\eta|<1.1$ & $0.1 \times 0.26$ & $18 X_{0}, 1 \lambda_{I}$ & $\frac{\sigma}{E_{T}}=\frac{13.5 \%}{\sqrt{E_{T}}} \oplus 2 \%$ \\
CHA & $|\eta|<0.9$ & $0.1 \times 0.26$ & $4.7 \lambda_{I}$ & $\frac{\sigma}{E_{T}}=\frac{50 \%}{\sqrt{E_{T}}} \oplus 3 \%$ \\
WHA & $0.9<|\eta|<1.3$ & $0.1 \times 0.26$ & $4.7 \lambda_{I}$ & $\frac{\sigma}{E_{T}}=\frac{75 \%}{\sqrt{E_{T}}} \oplus 4 \%$ \\
PEM & $1.1<|\eta|<3.6$ & $(0.1-0.6) \times(0.13-0.26)$ & $23 X_{0}, 1 \lambda_{I}$ & $\frac{\sigma}{E}=\frac{16 \%}{\sqrt{E}} \otimes 1 \%$ \\
PHA & $1.2<|\eta|<3.6$ & $(0.1-0.6) \times(0.13-0.26)$ & $6.8 \lambda_{I}$ & $\frac{\sigma}{E}=\frac{80 \%}{\sqrt{E}} \otimes 5 \%$ \\
\hline \hline
\end{tabular}

Table 3.1: Summary of the characteristics of the CDF Run II calorimeters.

\subsubsection{Shower Profile detectors}

The central and forward parts of the calorimeter have their own shower profile detector positioned at the expected maximum of the lateral shower profile, approximately at $6 X_{0}$. These Central (CES) and Plug Electromagnetic Showermax (PES) chambers [39] are designed to measure the position of photon and electron showers and to separate single photons from the photons produced in $\pi^{0} \rightarrow \gamma \gamma$ decays. The CES measures the charge deposition on orthogonal strips and wires. Cathode strips running in the azimuthal direction provide $z$ information, while anode wires running in the $z$ direction provide $r-\phi$ information. The position resolution of the CES for $50 \mathrm{GeV}$ electrons is approximately $2 \mathrm{~mm}$ in each direction. 


\subsubsection{Pre-Radiator detectors}

The Central Pre-Radiatior (CPR) and the Plug Pre-Radiator (PPR) [40] are located in the inner face of the central and plug calorimeters, between the calorimeter and the solenoid. The CPR is a set of four multi-wire proportional chambers, two at each side of $z=0$, positioned at a radius of $162 \mathrm{~cm}$ from the beam line. The CPR samples the early shower development in the solenoid, and was used in previous photon analyses at CDF for determining the background contamination from mesons [5] (more details on how it is used are given in Section 5.1).

\subsubsection{EMTiming system}

The EMTiming system [45] is installed in the CEM and PEM calorimeters and measures the time of arrival of the particles with a resolution higher than $1 \mathrm{~ns}$. Its design is optimized for high $E_{T}$ photons, and it is shown schematically in Fig. 3.8.

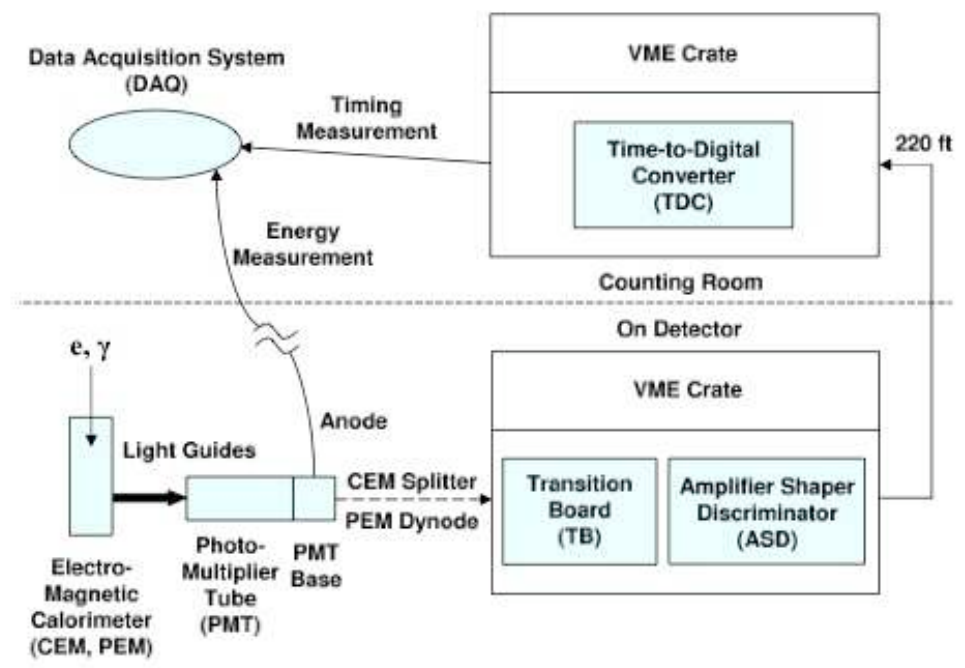

Figure 3.8: The EMTiming system layout.

The EMTiming system routes a copy of the PMT signal to a passive Transition Board (TB) and an Amplifier-Shaper-Discriminator board (ASD) that, in turn, converts analog signal into digital and sends it to a Time-to-Digital Converter (TDC) board for a timing measurement. The time of arrival of a particle is recorded if its deposited energy is above $3.8 \mathrm{GeV}$ in the case of the CEM, 
and $1.0 \mathrm{GeV}$ in the case of the PEM. The recorded time is corrected to remove channel-to-channel differences and to eliminate the dependence on the particle energy.

\subsubsection{Muon detectors}

The CDF II muon system [41] consists of four subsystems, the central muon chambers (CMU), the central muon upgrade chambers (CMP), the central muon extension (CMX) and the intermediate muon system (IMU). They are all functionally similar and combined cover the region of $|\eta|<2$ and $2 \pi$ in azimuth. The muons chambers are located outside the calorimeter systems, as shown in Fig. 3.2, and use the calorimeter steel and the magnet return yoke as absorbers for showering particles. The systems consists of drift cells and scintillator counters which are used to reconstruct the tracks from minimum ionizing particles. These tracks are matched using dedicated algorithms with the silicon and the COT information in order to reconstruct the full trajectory of the muons.

\subsubsection{Trigger and DAQ systems}

The collision rate at the Tevatron, with an average of $1.7 \mathrm{MHz}$, is much higher than the rate at which data can be stored on tape. The role of the trigger is to efficiently extract the most interesting physics events from the large number of $p \bar{p}$ collisions. The CDF trigger system has a three level architecture as shown in Fig. 3.9. Each level provides a rate reduction sufficient to allow for processing in the next level with minimal dead time.

Level 1 (L1) uses designed hardware to make decisions based on simple physics quantities using a subset of the detector information. As shown in Fig. 3.10, three different streams of information allow L1 to make a decision: calorimeter objects that may be further reconstructed into electrons, photons or jets; track segments in the muon detector; and tracking data to identify tracks which can be linked to objects in the calorimeter or muon detector. The L1 trigger decision takes place $5.5 \mu \mathrm{s}$ after a collision and it works in parallel through a pipeline that can store up to 14 bunch crossings. After L1, the event rate is reduced to less than $50 \mathrm{kHz}$.

The level 2 (L2) is a combination of hardware and software triggers that perform limited event reconstruction using programmable processors. These events are stored in one of four asynchronous buffers and the decision whether they are accepted or not is based on calorimeter cluster algorithms, shower information from Showermax (CES/PES) detectors and combined tracking information from L1 and from SVX II, which is crucial in order to trigger on different tracking 


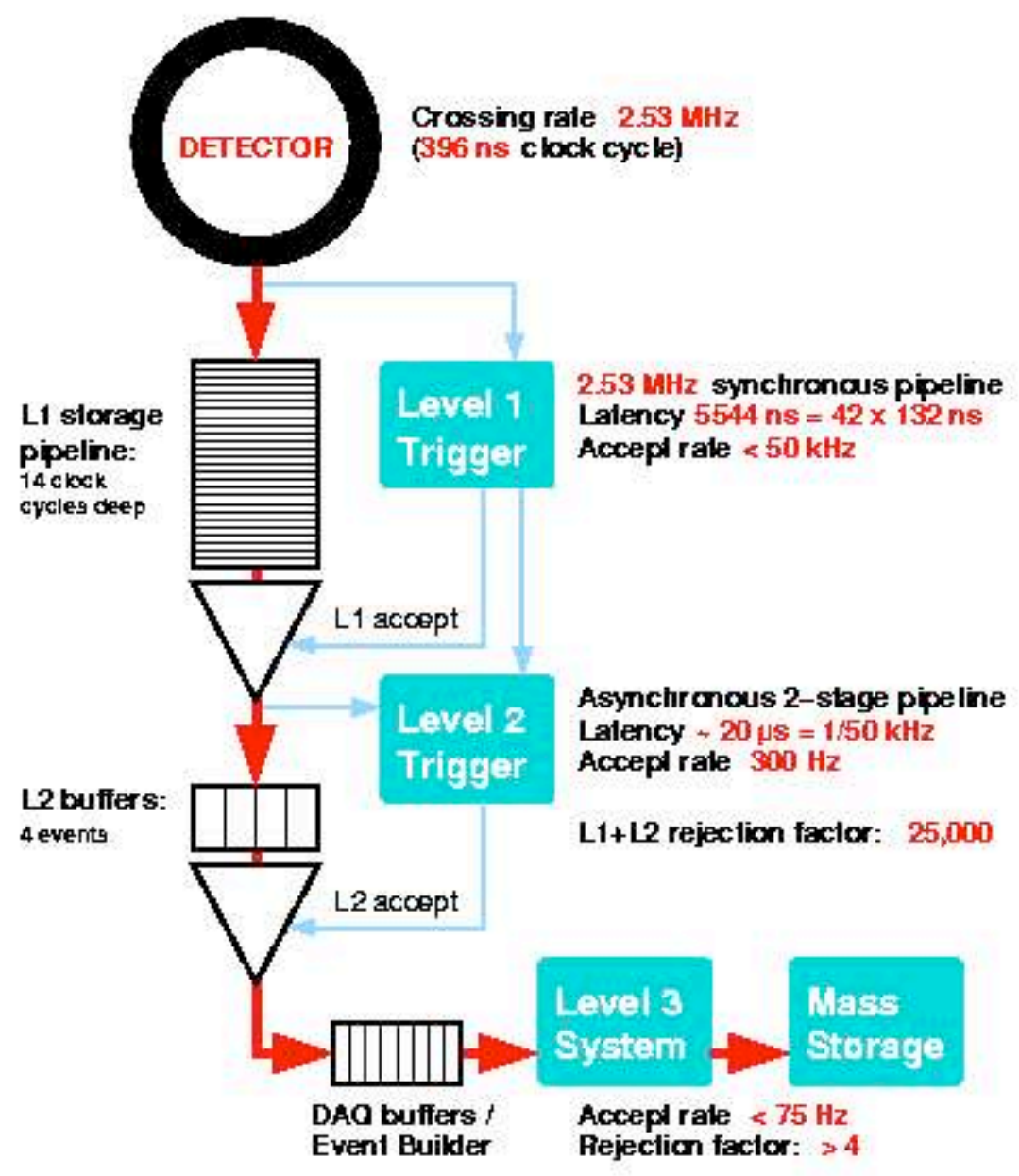

Figure 3.9: General diagram of CDF Run II trigger and data acquisition systems.

features like the impact parameter and to detect secondary vertexes from $B$ hadron decays. This level of decision takes approximately $20 \mu \mathrm{s}$ and further reduces the event rate to approximately $300 \mathrm{~Hz}$.

The level 3 (L3) consists of two components: an "event builder" and a Linux PC farm. As shown in Fig. 3.11 the detector readout from the L2 buffers is received via an Asynchronous Transfer Mode (ATM) switch and distributed to 16 PC nodes. The main task of these nodes is to assemble all the pieces of the same event as they are delivered from different subdetector systems 


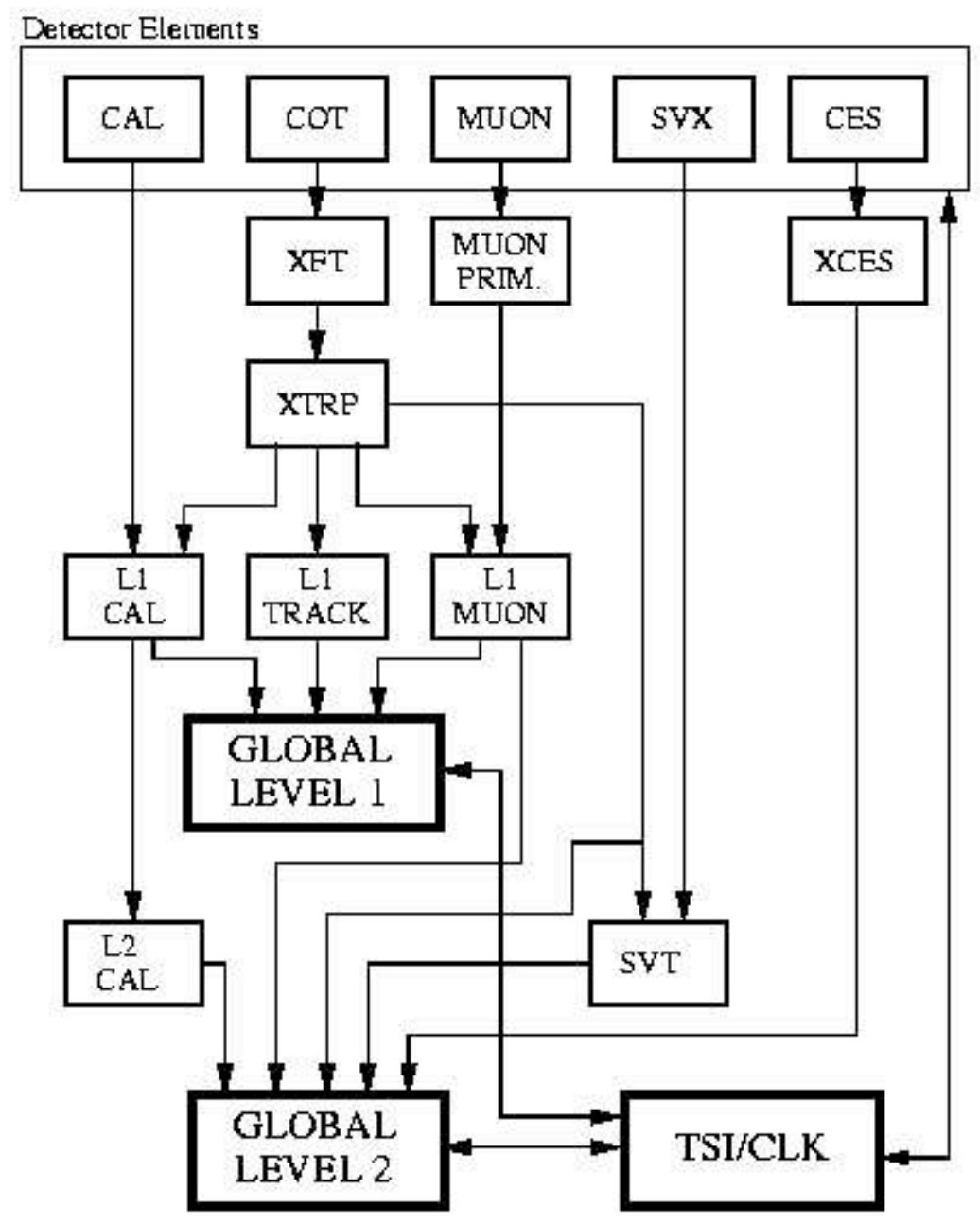

Figure 3.10: Diagram of Level 1 and Level 2 trigger systems and the data flow between them.

through the ATM switch. The event is then passed to a processor node consisting on a separate dual-processor PC. There are about 150 processor nodes and each of the two CPUs processes a single event at a time. The L3 decision is based on a near-final quality reconstruction which, if it passes certain criteria, it is sent to the Consumer Server / Data Logger (CS/DL) system for storage first on disk and then on tape. This level of decision reduces the latency to approximately $75 \mathrm{~Hz}$. 


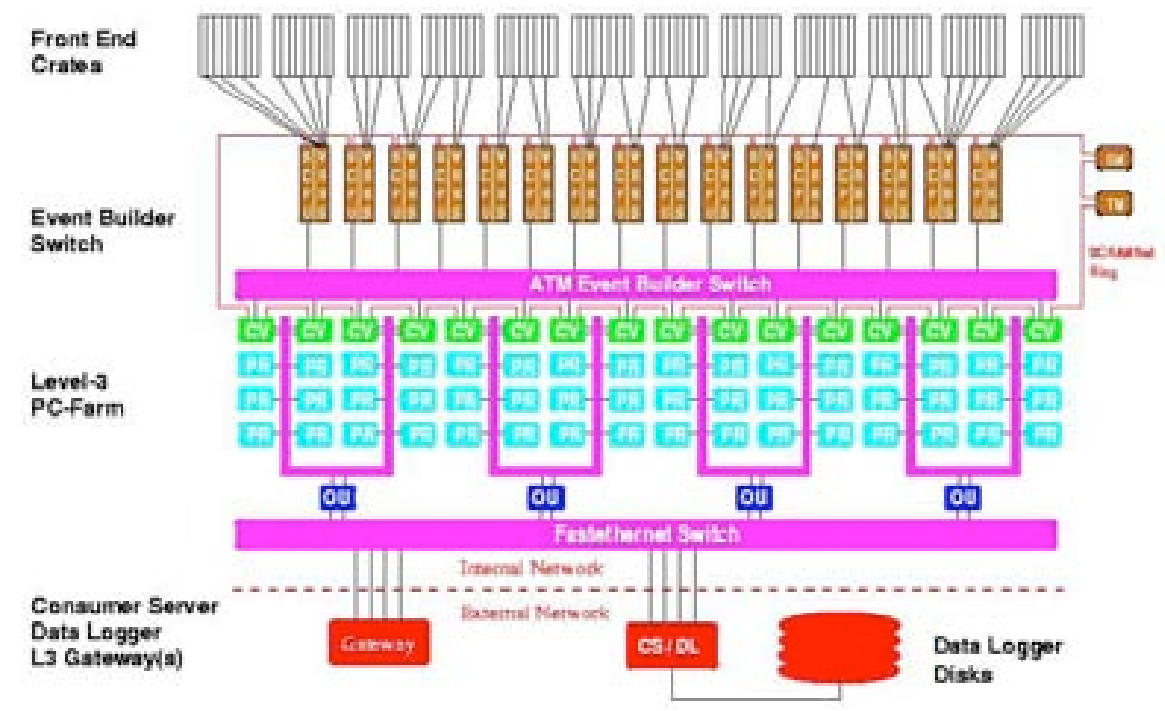

Figure 3.11: Event Builder and L3 filtering. Data from the front end crates pass through ATM switches to the converter nodes. Here, the events are assembled and passed to the processor nodes. The accepted events are passed to output nodes which send them to the Consumer Server and Data Logging systems (CS/DL).

\subsubsection{Luminosity measurement}

The instantaneous luminosity $(\mathcal{L})$ at $\mathrm{CDF}$ is determined from the rate of inelastic $p \bar{p}$ interactions in the Cherenkov Luminosity Counters (CLC) [42] detector. The CLC is formed by two gas modules which occupy the conical holes $(3.75<|\eta|<4.75)$ of $3^{\circ}$ between the plug calorimeters and the beam pipe, as shown in Fig. 3.12. Each module contains 48 long conical gas-filled Cherenkov counters pointing to the center of the detector and is equipped with PMT tubes. The CLC measures the average number of inelastic interactions per bunch crossing, $\mu$, within a few percent and up to the highest instantaneous luminosities experienced at the Tevatron. Since the number of interactions $n$ per bunch crossing follows Poisson statistics with mean $\mu$, a good estimator for $\mu$ can be obtained by measuring the fraction of empty bunch crossings, $N_{n=0}$, over the total number of crossings, $N_{\text {total }}$ :

$$
\mathcal{P}(n=0)=e^{-\mu}=\frac{N_{n=0}}{N_{\text {total }}}
$$

An empty bunch crossing is observed when there are less than two PMT tubes with signals above threshold in either module of the CLC. The measured fraction of empty bunch crossings is cor- 
rected for the CLC acceptance, $\varepsilon_{C L C}$, and the value of $\mu$ is calculated. Then, the instantaneous luminosity is extracted using:

$$
\mu f_{B C}=\sigma_{i} \varepsilon_{C L C} \mathcal{L}
$$

where $\sigma_{i}=59.3 \mathrm{mb}$ is the inelastic $p \bar{p}$ scattering cross section, $f_{B C}$ is the frequency of bunch crossing, which is on average $1.7 \mathrm{MHz}$ for $36 \times 36$ bunch operations, and $\varepsilon_{C L C}=0.60 \pm 0.03$. The uncertainty on the luminosity is about $6 \%$, which originates from uncertainties in the acceptance $(4.4 \%)$ and from the inelastic cross section normalization (4\%).

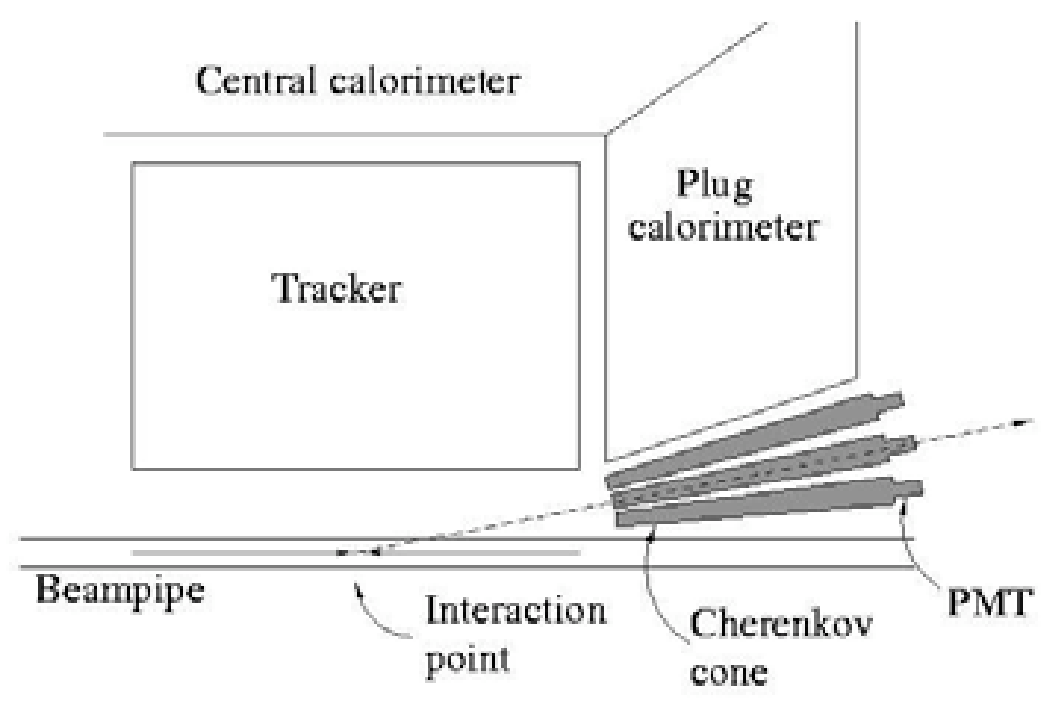

Figure 3.12: Schematic view of the luminosity monitor inside a quadrant of CDF. It is located at $|\theta|<3^{\circ}$.

\subsection{Offline Photon Reconstruction}

A photon cluster is formed by 1 to 3 adjacent towers in $\eta$ in the calorimeter. Photon and electron candidates are reconstructed starting from "seed" calorimeter towers with transverse electromagnetic energy $E_{T, E M}>3 \mathrm{GeV}$. In the central calorimeters, electromagnetic clusters must be contained in the same wedge in $\phi$ and at most can consist of the seed and its nearest neighbours. Neighbour towers must satisfy $E_{T}>100 \mathrm{MeV}$. Every tower can only be in one cluster. In the plug calorimeters, EM clusters have a square $2 \times 2$ tower configuration. 
EM clusters are treated as massless objects. The total energy of the cluster is $E=E_{E M}+E_{H A D}$, where $E_{E M}$ and $E_{H A D}$ are the sum of the corresponding electromagnetic and hadronic energies. The transverse energy of the photon is obtained from the energy after correcting it for the position of the photon (measured in the CES detector) as relative to the hard interaction vertex, given by the polar angle $\theta$ :

$$
E_{T}=E \sin \theta
$$

The $(\eta, \phi)$ coordinates of the cluster are determined following the snowmass scheme:

$$
\begin{aligned}
\eta & =\frac{E_{E M} \times \eta_{E M}+E_{H A D} \times \eta_{H A D}}{E} \\
\phi & =\frac{E_{E M} \times \phi_{E M}+E_{H A D} \times \phi_{H A D}}{E}
\end{aligned}
$$

where $\eta_{E M}$ and $\phi_{E M}$ are pondered over the towers in the cluster:

$$
\begin{aligned}
& \eta_{E M}=\frac{\sum_{i} E_{E M}^{i} \times \eta^{i}}{\sum_{i} E_{E M}^{i}} \\
& \phi_{E M}=\frac{\sum_{i} E_{E M}^{i} \times \phi^{i}}{\sum_{i} E_{E M}^{i}}
\end{aligned}
$$

and, similarly, $\eta_{H A D}$ and $\phi_{H A D}$ are obtained according to:

$$
\begin{aligned}
\eta_{H A D} & =\frac{\sum_{i} E_{H A D}^{i} \times \eta^{i}}{\sum_{i} E_{H A D}^{i}} \\
\phi_{H A D} & =\frac{\sum_{i} E_{H A D}^{i} \times \phi^{i}}{\sum_{i} E_{H A D}^{i}}
\end{aligned}
$$

The EM cluster transverse energy is then required to be greater than $5 \mathrm{GeV}$, and the ratio of the energy of the hadronic clusters to that of the EM clusters must be less than 0.125 if $E_{T}^{E M}<100 \mathrm{GeV}$. Photons are identified from EM clusters based on information of the lateral profile of the shower, the energy deposition in the towers around the photon cluster, and the the $p_{T}$ of the tracks pointing to the cluster, among others. The set of variables used for the photon selection and identification is explained in detail in the next Chapter. 



\section{Chapter 4}

\section{The Inclusive Isolated Prompt Photon Cross Section Measurement}

In this chapter we will describe in detail the measurement of the inclusive prompt photon cross section for isolated and central photons. The chapter begins with the description of the dataset used and the Monte Carlo samples employed in the analysis, followed by the event selection criteria. Most of the chapter is devoted to the explanation of the background suppression method and to the determination of the photon purity. Finally, the procedure to unfold the measurement for detector effects back to the hadron level is described. The discussion on the systematic uncertainties will be treated separately in Chapter 5 .

\subsection{Data Samples}

We use the data collected between February 2002 and August 2007 which correspond to a total integrated luminosity of $2.5 \mathrm{fb}^{-1}$ and provide sufficient statistics up to $p_{T}=400 \mathrm{GeV} / \mathrm{c}$. Data are selected using three high $p_{T}$ photon triggers, the PHOTON_25_ISO trigger for energies between 30 and $90 \mathrm{GeV}$, and a logical OR of two different paths, SUPER_PHOTON70_JET and SUPER_PHOTON70_EM, for $p_{T}>90 \mathrm{GeV} / \mathrm{c}$. Tables 4.1 and 4.2 summarize the trigger cuts. The triggers selection requires a cluster of energy in the central electromagnetic calorimeter $(|\eta|<1.1)$ and small energy depositions in the associated hadronic calorimeter towers. To reduce the number of low $p_{T}$ photons coming from meson decays, the low $p_{T}$ trigger only selects isolated photons 
in the calorimeter. For higher energies the production rate of light mesons is not so important and no isolation is needed in order to avoid the saturation of the trigger. The PHOTON_25_ISO and the SUPER_PHOTON70_EM triggers require small HAD/EM at L2 to remove jets from the sample, which becomes inefficient at $p_{T}>130 \mathrm{GeV} / \mathrm{c}$ due to the saturation of the $\mathrm{L} 2$ readout electronics (see Section 4.5). Since the SUPER_PHOTON70_JET trigger does not include any HAD/EM cut at L2, an OR of SUPER_PHOTON70_EM and SUPER_PHOTON70_JET serves to recover this inefficiency at high $p_{T}$. This logical OR is referred as SUPER_PHOTON70 trigger.

\begin{tabular}{l|l|l}
\hline \hline \multicolumn{2}{l|}{ Trigger level } & PHOTON_25_ISO \\
\hline L1 & $E_{T}$ & $>8.0$ \\
\hline \multirow{4}{*}{ L2 } & $E_{T}$ & $>21.0$ \\
& $\mathrm{HAD} / \mathrm{EM}$ & $<0.125$ \\
& Iso & $<3.0 \|<0.15 E_{T}$ \\
\hline & $E_{T}$ & $>25.0$ \\
& $\mathrm{CES} \chi^{2}$ & $<20.0$ \\
L3 & $\mathrm{HAD} / \mathrm{EM}$ & $<(0.055+0.00045) E$ (if $E<200)$ \\
& & $<0.2+0.001 E$ (if $E>200)$ \\
& Iso & $<0.10 E_{T}$ \\
\hline \hline
\end{tabular}

Table 4.1: The different level requirements of the PHOTON_25_ISO trigger, applied for $30<p_{T}^{\gamma}<90 \mathrm{GeV} / \mathrm{c}$. Energies are in $\mathrm{GeV}$.

\begin{tabular}{l|l|ll}
\hline \hline \multicolumn{2}{l|}{ Trigger level } & SUPER_PHOTON70_EM & SUPER_PHOTON70_JET \\
\hline L1 & $E_{T}$ & $>8.0$ & $>10.0$ \\
\hline & $E_{T}$ & $>70.0$ & $>70$ \\
L2 & HAD/EM & $<0.125$ & - \\
\hline L3 & $E_{T}$ & $>70.0$ & $>70.0$ \\
\hline \hline
\end{tabular}

Table 4.2: The different requirements for all the levels of the SUPER_PHOTON70_EM and the SUPER_PHOTON70_JET triggers, applied for $p_{T}^{\gamma}>90 \mathrm{GeV} / \mathrm{c}$. The SUPER_PHOTON70 trigger path is an OR of these two. Energies are in GeV.

In this measurement, a sample of high $p_{T}$ electrons from $Z$ decays is used to determine the PHOTON_25_ISO trigger efficiency and to quantify the systematic uncertainty in the photon purity. 
This sample also serves to set the correction to the photon absolute energy scale, and to study the photon selection efficiency. The electron data was collected by the ELECTRON_CENTRAL_18 trigger in the same periods as the inclusive photon sample. This high $p_{T}$ electron trigger requires a cluster of energy in the central electromagnetic calorimeter and a track pointing to it. To determine the efficiency of the SUPER_PHOTON70 trigger we use a inclusive jet sample, collected with the JET_100 trigger, which only requires a calorimeter tower of $10 \mathrm{GeV}$ at L1.

For the measurement, we only consider data for which all the subsystems relevant for the analysis are fully operational. These are the COT, the Calorimeters and the CES detector.

\subsection{Monte Carlo Samples}

Photon Monte Carlo samples are used to estimate the efficiency of the ID cuts and to unfold the measurement back to the hadron level. The technique employed for the background estimation uses the isolation distributions obtained from both Monte Carlo photon and dijet samples (see Section 4.6), while to determine the effect of the underlying event in the cross section at parton level, different tunings of the underlying event model, Tune A and Tune DW, are used in the analysis. A $Z\left(\rightarrow e^{+} e^{-}\right)$Monte Carlo sample serves to study the energy scale in the photon Monte Carlo and for setting the systematics in the photon absolute energy scale in the data sample.

All the used Monte Carlo samples are PYTHIA v6.216 with CTEQ5L parton distribution functions [43] and the Tune A parametrization set for the underlying event [29]. The events are passed through a GEANT-based CDF detector simulation to reproduce the detector response [26]. The generation of the Monte Carlo has been done for different $\hat{p_{T}}$ ranges to guarantee enough statistics along the $p_{T}$ range considered in this measurement.

\subsection{Event Selection}

Every object in data and Monte Carlo must pass the same trigger and offline selection. In the case of electrons, the photon ID cuts are adapted to allow a track, while the jets are always selected with the same cuts applied to the photon samples.

Photon (or electron) candidates are matched to a L2 and a L3 trigger cluster and are required to pass the PHOTON_25_ISO trigger for $p_{T}$ less than $90 \mathrm{GeV} / \mathrm{c}$ or the SUPER_PHOTON70 trigger for higher photon $p_{T}$. In the Monte Carlo, we require them to pass the simulation of that triggers. 
This is specially important for the low $p_{T}$ region, where the isolation cut in the trigger sculpts the shape of the high tail of the isolation distribution in the templates.

\subsubsection{Photon selection variables}

The photon triggers do not collect only photons but also a considerably amount of background. Photons coming from meson decays may survive the isolation cut at the trigger level; since no tracking requirements are applied, the photon dataset may also contain electrons; and cosmic muons may radiate a photon which passes the trigger cuts. The different offline requirements are designed to specifically suppress these different types of backgrounds. They increase the purity of the photon data sample but are not enough to totally remove the photons from mesons. The suppression of these photons will be treated in detail in Section 4.6. Now we list all the different variables used to select the photon signal. The different requirements are shown in Table 4.3. The photon ID efficiency is computed using photon Monte Carlo samples and will be discussed together with other corrections in Section 4.7.

\section{- The CES Fiducial}

The CES fiducial cuts are imposed to avoid uninstrumented regions at the edges of the CES detector. The CES $x$ position must be within $21 \mathrm{~cm}$ from the center of the detector, while the CES $z$ position is required to be less than $200 \mathrm{~cm}$ away from the geometrical center of the detector.

\section{- CES strips and wire clusters requirement}

The information from the CES is used to reconstruct the lateral shower profile of the photon and to measure its position in the detector. To be selected, the photon is required to have a cluster in both strip and wire layers. The CES cluster is formed by the highest $E_{T}$ (seed) strip (wire) and the hits within a 11-strip (wire) window around the seed, where the highest $E_{T}$ strip (wire) cluster must be within $25 \mathrm{~cm}$ in $z$ of the EM centroid. This requirement ensures there is information enough to apply other cuts in derived quantities.

\section{- Energy}

All photon candidates are required to have corrected $E_{T}>30 \mathrm{GeV}$. The details on the corrections applied to the measured energy are given in Section 4.4. Since photons are massless objects, $E_{T}=p_{T}$.

\section{- HAD/EM}

$\mathrm{HAD} / \mathrm{EM}$ is the ratio of the total hadronic calorimeter energy to the total electromagnetic 
energy for the photon cluster towers. This ratio is required to be small to reject background from jets, which will have high energy depositions in the hadronic calorimeter. At high photon $p_{T}$, the cut is variable depending on $p_{T}^{\gamma}$ in order to account for possible leakage of the photon shower in the hadron calorimeter.

\section{- The calorimeter isolation}

The isolation transverse energy in the calorimeter is defined as the transverse energy in a cone of $R=\sqrt{\Delta \eta^{2}+\Delta \phi^{2}}=0.4$ centered in the photon cluster centroid minus the energy of the photon itself:

$$
E_{T}^{i s o}=E_{T}(R=0.4)-E_{T}^{\gamma}
$$

The isolation for direct photons comes from the underlying event energy and from the pile-up. In the case of photons coming from fragmentation or from meson decays, the hadronic particles produced with the photon may also contribute to the isolation. There are other contributions due to detector effects: since the EM clustering is done only in the $\eta$-direction, energy which leaks out of the shower laterally into the adjacent wedge can be included in the isolation cone but lost from the shower. This contribution depends on the energy of the photon and the position of the shower, given by the CES $x$ position (for more details see Appendix C).

No cut in the calorimeter isolation is required for the photon candidates in the templates to allow for the fitting of the isolation distribution, but all data and Monte Carlo photon candidates are required to pass the trigger cuts, which for low $p_{T}$ (PHOTON_25_ISO) include an isolation cut $\left(E_{T}^{i s o} / E_{T}^{\gamma}<0.10\right)$ that sculpts the shape of the tail in the low $p_{T}$ isolation templates. For the event yield that goes into the cross section we require $E_{T}^{i s o}<2 \mathrm{GeV}$.

\section{- $\operatorname{CES} \chi^{2}$}

The CES detector is used to measure the lateral shower shape of the photon candidates in the event. The measured shower shape is compared to the expected shower shape for a single photon, obtained from test beam. The consistency between these two showers is quantified with a $\chi^{2}$ parameter, the "CES $\chi^{2}$ ". The CES $\chi^{2}$ is computed for every strip (wire) layer by comparing the energy in the 11 strips (wires) of the CES strip (wire) cluster to what we expect from a single shower obtained from electrons in a test beam experiment, taking into account the estimated variance of the electron profile. After calculating the $\chi^{2}$ for each CES plane, the final $\chi^{2}$ of the fit is defined as the average $\chi^{2}=\left(\chi_{\text {wire }}^{2}+\chi_{\text {strip }}^{2}\right) / 2$. If the obtained value for the photon candidate is below 20, then its shower shape is considered compatible with that expected for a single photon and the photon candidate passes the cut. 
If not, then the photon candidate may come from a meson decay and it is rejected.

In this measurement, the CES $\chi^{2}$ requirement is only applied for photons with $p_{T}<90 \mathrm{GeV} / \mathrm{c}$, since the efficiency of this cut at high $p_{T}$ is not well reproduced by the Monte Carlo samples [44]. Measurements with data show that the CES $\chi^{2}$ efficiency decreases at high $p_{T}$, while Monte Carlo predicts a flatter behaviour. For $30<p_{T}<90 \mathrm{GeV} / \mathrm{c}$ the cut is present in the trigger requirements (see Table 4.1), so it cannot be completely removed from the selection. The $\mathrm{L} 3 \chi^{2}$ cut is very similar to that offline, the only difference being the correction for the primary vertex position, and therefore we choose to apply this requirement at offline level too. In this $p_{T}$ range, the CES $\chi^{2}$ efficiency is obtained from the photon Monte Carlo and compared to that from electrons in a $Z$ data sample. The uncertainties due to this choice will be discussed more in Chapter 5 .

\section{- 2nd CES cluster}

Photon pairs from meson decays may not be resolved by the calorimeter. However, although detected as a single photon, a fraction of them might be separated enough so that a 2nd CES cluster is observed near to the primary one. Therefore, a cut on the energy deposited in the second CES cluster can further remove meson background contributions. Events that do not present a 2nd cluster pass the selection. If only either the strips or wires have a second cluster, the cut is placed on the available one.

\section{- $\mathbf{N}$ tracks and Track $p_{T}$}

$N_{t r k}$ is the number of tracks which hit the calorimeter within $5 \mathrm{~cm}$ of center of the photon cluster. One soft track is allowed in order to account for underlying event activity, but the $p_{T}$ of this track has to be of the order of 1-2 GeV depending on the photon $p_{T}$ (see Table 4.3). This cut is necessary to remove electrons from the data sample.

\section{- $\mathrm{MET} / E_{T}^{\gamma}$}

To remove the cosmic background we apply MET/ $E_{T}^{\gamma}<0.8$. The reasons for this choice and the efficiency of this cut are discussed in Section 4.6.1.

To maintain the projective geometry of the calorimeter towers, all events are required to have a well reconstructed primary vertex within $60 \mathrm{~cm}$ around the center of the detector.

\subsubsection{Photon-like electron identification}

We use electrons from $Z$ decays in data and Monte Carlo samples to determine the systematic uncertainty in the photon purity, the trigger efficiency, and the correction of the photon absolute 


\begin{tabular}{ll}
\hline \hline Variable & Value \\
\hline Central & Yes \\
corrected $E_{T}$ & $>30.0 \mathrm{GeV}$ \\
$\mathrm{HAD} / \mathrm{EM}$ & $\leq 0.055+0.00045 \cdot E$ \\
Strip and Wire average CES $\chi^{2}$ & $\leq 20\left(\right.$ if $\left.E_{T}<90 \mathrm{GeV}\right)$ \\
$\left|X_{C E S}\right|$ & $\leq 21 \mathrm{~cm}$ \\
$\left|Z_{C E S}\right|$ & $9-200 \mathrm{~cm}$ \\
$\mathrm{~N}_{t r k}$ & $\leq 1$ \\
$p_{T}^{\text {trk }}$ & $<1+0.005 \cdot E_{T}$ \\
$2^{\text {nd }} E_{C E S}$ & $<0.14 \cdot E_{T}$ (if $\left.E_{T}<18\right)$ \\
& $<2.4+0.01 \cdot E_{T}\left(\right.$ if $\left.E_{T}>18\right)$ \\
\hline \hline
\end{tabular}

Table 4.3: The applied photon cuts. Energies are in $\mathrm{GeV}$

energy scale. To reconstruct the $e^{+} e^{-}$invariant mass, we select both tight and loose electrons, but only the tight ones are used for the different studies. Tight electrons are identified with the photon cuts described in Section 4.3.1, modified to allow a track (see Table 4.4), while we require loose electrons to pass only fiducial and HAD/EM cuts. Then, tight-tight and tight-loose pairs must have an invariant mass $M_{Z}$ such as $81<M_{Z}<101 \mathrm{GeV} / \mathrm{c}^{2}$. To further reject bremsstrahlung all electrons are required to have $0.8<E / p<1.2$, where $E$ is the electron energy measured in the calorimeter and $p$ the momentum of the electron measured in the tracking detector.

\subsection{The photon energy scale}

The transverse energy of the EM objects (electrons and photons) in the CEM is corrected to account for non uniformities in the calorimeter response [47]:

\section{- Correction for time-dependent gain variation}

The gain of each phototube of the CEM is monitored as a function of the time during a collider run in order to detect long-term stability fluctuations. The long-term stability of other detection components such as the WLS, the light guides and the scintillators is also taken into account for measuring the degradation of the energy resolution and the energy scale with the time. 


\begin{tabular}{lll}
\hline \hline Variable & Tight & Loose \\
\hline Central & Yes & Yes \\
corrected $E_{T}$ & $>30$ & $>30$ \\
$\mathrm{HAD} / \mathrm{EM}$ & $\leq 0.055+0.00045 \cdot E$ & $\leq 0.055+0.00045 \cdot E$ \\
Strip and Wire average CES $\chi^{2}$ & $\leq 20$ if $E_{T}<90 \mathrm{GeV}$ & - \\
$\left|X_{C E S}\right|$ & $\leq 21 \mathrm{~cm}$ & $\leq 21 \mathrm{~cm}$ \\
$\left|Z_{C E S}\right|$ & $9-200 \mathrm{~cm}$ & $9-200 \mathrm{~cm}$ \\
$\mathrm{~N}_{t r k}$ & $\leq 2$ & - \\
$2^{\text {nd }}$ Track $p_{T}$ & $>1+0.005 \cdot E_{T}$ & - \\
$2^{\text {nd }} E_{C E S}$ & $<0.14 \cdot E_{T}\left(\right.$ if $\left.E_{T}<18\right)$ & - \\
& $<2.4+0.01 \cdot E_{T}\left(\right.$ if $\left.E_{T}>18\right)$ & - \\
$\mathrm{E} / \mathrm{p}$ & $0.8-1.2$ & $0.8-1.2$ \\
\hline \hline
\end{tabular}

Table 4.4: The applied photon-like electron cuts. Energies are in GeV.

\section{- Correction for response variations in the individual CEM tower}

The calorimeter response depends on whether the particle hits in the center or in the edges of the tower. The correction factors due to this effect were obtained from electrons in test beam experiments. Ref. [46] describes the results from $50 \mathrm{GeV}$ electron data.

\section{- Wedge-to-wedge correction}

Due to the tower clustering only in the $\eta$ direction, energy which leaks out of the shower laterally into the adjacent wedge is lost from the shower. The amount of energy lost depends on the position of the shower in the CES and energy of the shower.

In addition to these corrections, we scale the energy of the photons in data and in Monte Carlo using electrons from $Z$ decays. The invariant $Z$ mass obtained from the decay is fitted to a double Gaussian (signal) plus a second-order polynomial (background). An example of a fit for one bin in $p_{T}$ is shown in Fig. 4.1. The means and the widths of the two Gaussians are not fixed, and the $Z$ mass is given by the mean of the narrower Gaussian ${ }^{1}$. The ratio of the reconstructed $Z$ mass to that in the PDG $\left(91.1876 \mathrm{GeV} / \mathrm{c}^{2}\right)$ is the energy scale correction factor, which is time-dependent and increasing with luminosity for data, and constant for Monte Carlo. The average of the correction

\footnotetext{
${ }^{1}$ We have also simply fit the region near the $Z$ peak to a single Gaussian and obtained results which in general differ by $0.1 \mathrm{GeV} / \mathrm{c}^{2}$ and at most $0.4 \mathrm{GeV} / \mathrm{c}^{2}$.
} 
factor for data is about $0.994 \pm 0.0002$ (see Fig. 4.2); for Monte Carlo it is $1.0035 \pm 0.0001$.

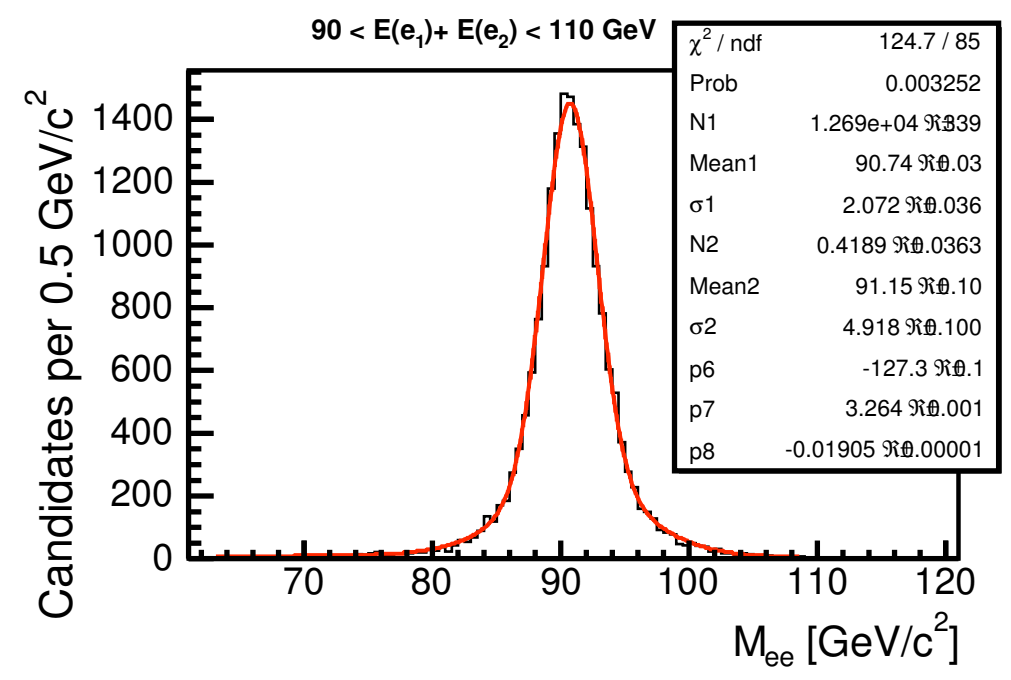

Figure 4.1: The invariant mass $M_{e e}$ distribution from $Z \rightarrow e^{+} e^{-}$data for the bin $90<E\left(e^{+}\right)+$ $E\left(e^{-}\right)<110 \mathrm{GeV}$. The curve indicates the result of the fit to double Gaussian and a second-order polynomial background.

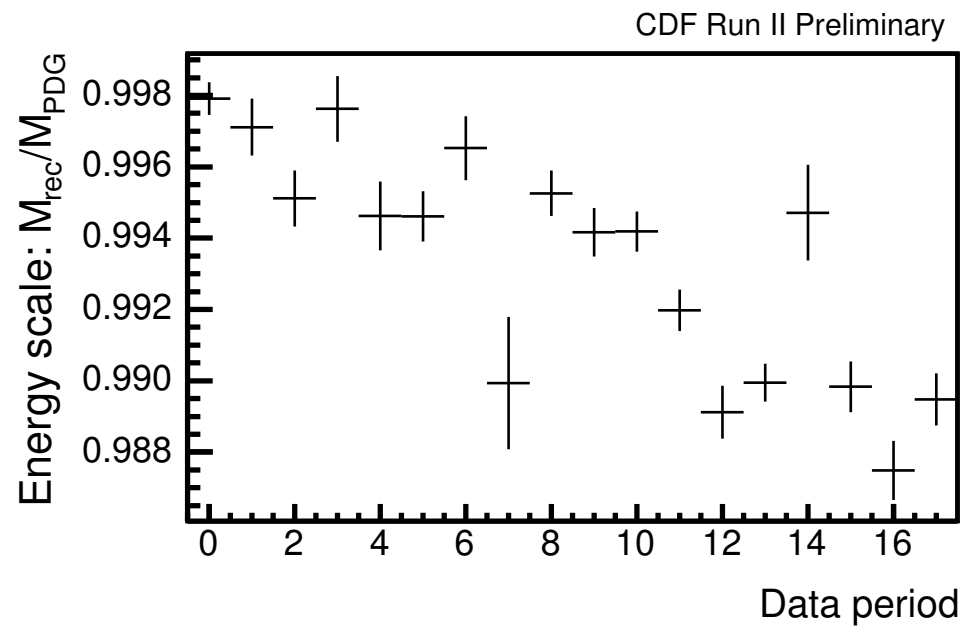

Figure 4.2: The energy scale correction from $Z$ 's $v s$ data period. Every data period corresponds to 2-5 months of data taking. The luminosities in each data period range from about $100 \mathrm{pb}^{-1}$ to $500 \mathrm{pb}^{-1}$. 


\subsection{Triggers Efficiency}

Two triggers are used in this measurement. For photons with $p_{T}<90 \mathrm{GeV}$ we use the PHOTON_25_ISO trigger sample. For photon energies above $90 \mathrm{GeV}$, the SUPER_PHOTON70 trigger (which is a logical OR of two different trigger paths, SUPER_PHOTON70_EM and SUPER_PHOTON70_JET) is used instead. See Section 4.1 for details on the trigger selection cuts.

\subsubsection{PHOTON_25_ISO trigger efficiency}

In order to measure the PHOTON_25_ISO trigger efficiency, we look for $Z \rightarrow e^{+} e^{-}$in the same data periods where the measurement is performed. The electron samples are collected with a high $p_{T}$ electron trigger which requires a high energy cluster in the central EM calorimeter and a track pointing to it. The events must pass the cuts described in Section 4.3, and the electron pairs are selected from the dataset using the cuts described in Section 4.3.2 and detailed in Table 4.4. For the construction of the invariant $Z$ mass, we also consider loose plug photon-like electrons, detected in the plug calorimeters and selected using photon variables adapted to allow a track. The electron pair is then required to have $81<M_{Z}<101 \mathrm{GeV} / \mathrm{c}^{2}$. The trigger efficiency is defined as the ratio between the number of electrons which are central and tight and the number of central tight electrons which also satisfy the PHOTON_25_ISO trigger requirements at level 1-3. The resulting efficiency is shown in Fig. 4.3, and in Table 4.5 for $p_{T}<90 \mathrm{GeV} / \mathrm{c}$.

The PHOTON_25_ISO trigger starts to become inefficient at about $130 \mathrm{GeV}$ due to the saturation of the $\mathrm{L} 2$ readout electronics, that leads to inefficiency in the L2 HAD/EM cut at $p_{T}^{\gamma} \sim 127.5 \mathrm{GeV} / \mathrm{c}$. The readout electronics of the L2 trigger towers in the central calorimeter can measure energies up to $\sim 127.5 \mathrm{GeV} / \mathrm{c}$, where it saturates. The hadronic part of the trigger tower does not saturate until higher energies, and therefore, its ratio with the EM energy artificially increases for energies higher than $\sim 127.5 \mathrm{GeV} / \mathrm{c}$, leading to important inefficiencies ${ }^{2}$ at high $p_{T}$. We have studied this effect using SUPER_PHOTON70 data, in which no HAD/EM $<0.125$ requirement is applied at the trigger level. The denominator is the number of photon candidates which satisfy the offline photon identification requirements (see Section 4.3). The numerator is the number of offline photon candidates which are matched to level 2 EM clusters with $\mathrm{HAD} / \mathrm{EM}<0.125$. The result of this study is shown in Fig. 4.4. The trigger efficiency drops by less than 3\% for energies below $200 \mathrm{GeV}$, while after $200 \mathrm{GeV}$ the loss of efficiency is faster and at $400 \mathrm{GeV}$ it is about $30 \%$.

\footnotetext{
${ }^{2}$ More details can be found in Ref. [49].
} 


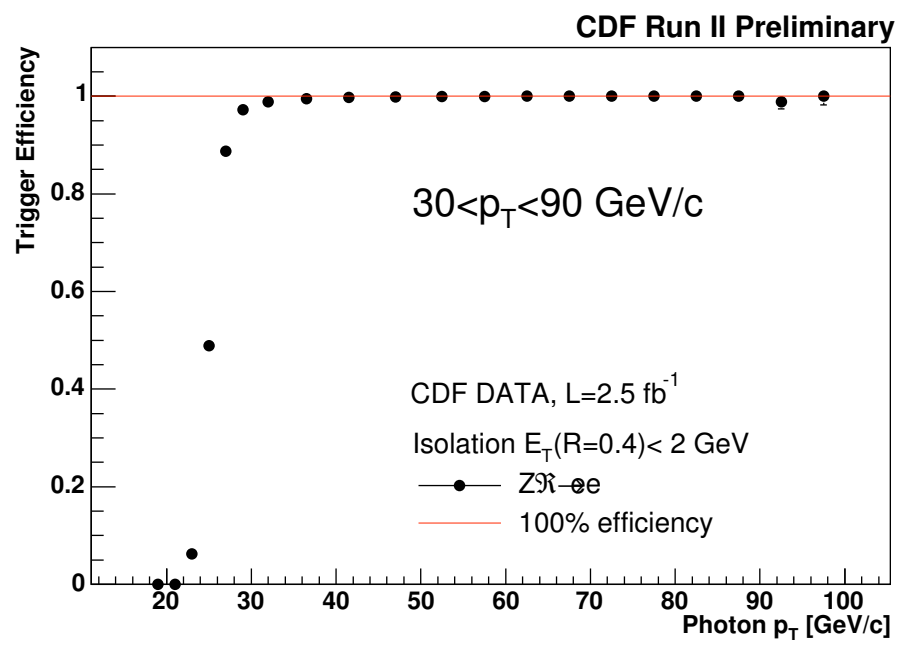

Figure 4.3: Efficiency of the PHOTON_25_ISO trigger as a function of the photon transverse momentum.

For this study, the background contributions of cosmics and light meson decays have not been removed from the data sample. The contamination of jet background is estimated to be $<5 \%$ for $p_{T}>100 \mathrm{GeV}$, as shown in [50], and the cosmic events tend to have very small HAD/EM, so they will not pull down the efficiency in the high $p_{T}$ region [51].

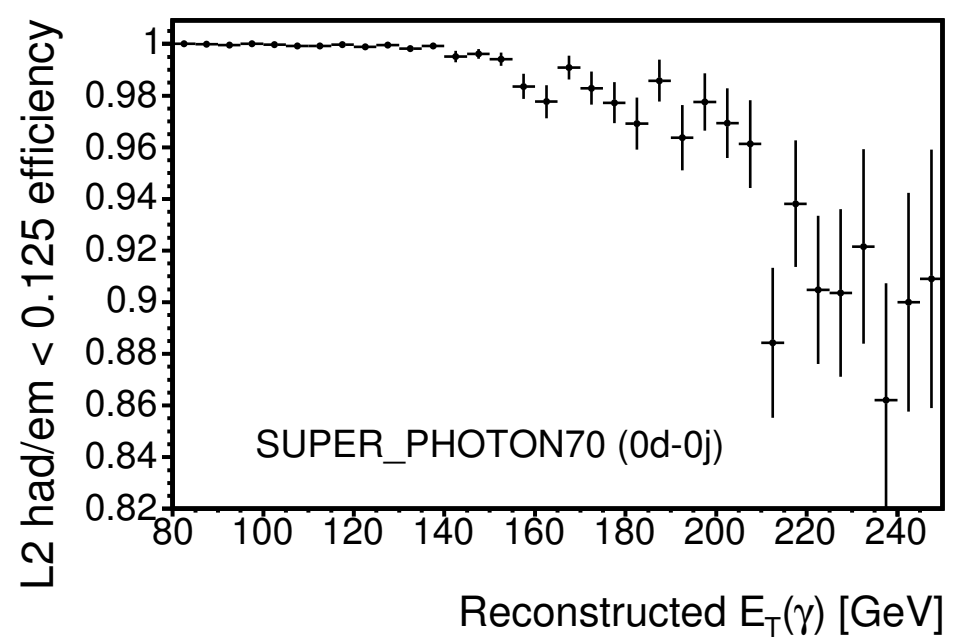

Figure 4.4: The efficiency of the level $2 \mathrm{HAD} / \mathrm{EM}<0.125$ requirement as a function of reconstructed photon $p_{T}$. The data are collected with the SUPER_PHOTON70 trigger. 


\subsubsection{SUPER_PHOTON70 trigger efficiency}

For $p_{T}<120 \mathrm{GeV}$ the $Z$ sample still provides sufficient statistics and can be used to measure the SUPER_PHOTON70 trigger efficiency in the same way it was used for the PHOTON_25_ISO. For higher $p_{T}$ 's, the efficiency is measured using the JET_100 sample described in Section 4.1 and can be expressed as:

$$
\varepsilon_{\text {trig }}=\frac{N_{\text {photons }+ \text { trig }}}{N_{\text {photons }}}
$$

where $N_{\text {photons }}$ is the number of photon candidates selected with the photon selection described in Section 4.3.1 and $N_{\text {photons }+ \text { trig }}$ is the number of photon candidates which also pass the trigger cuts. As shown in Fig. 4.5 and in Table 4.5 for $p_{T}>90 \mathrm{GeV} / \mathrm{c}$, the trigger is $100 \%$ efficient for photon $p_{T}$ 's above $90 \mathrm{GeV}$, where it is started to be used.

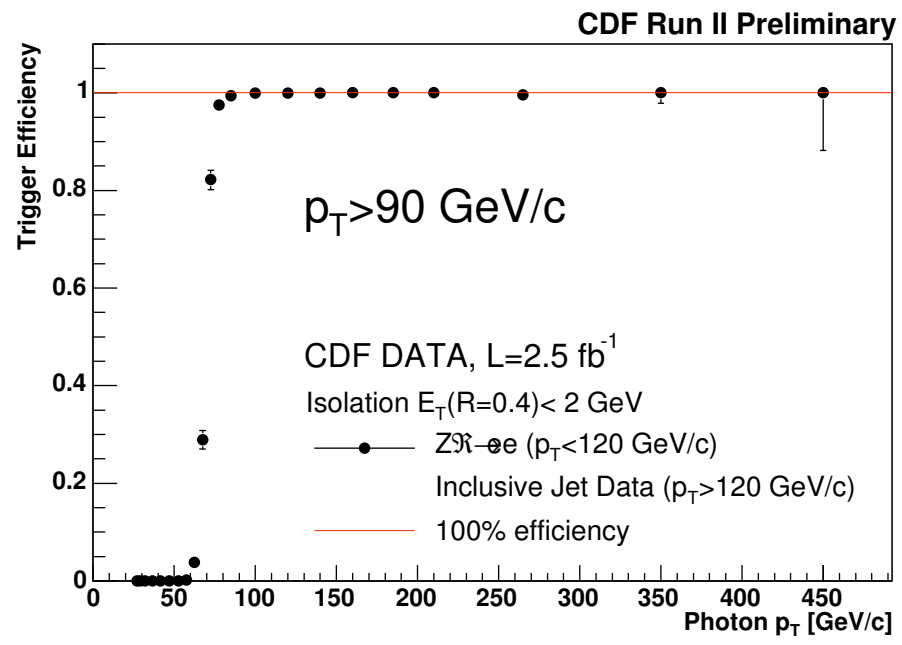

Figure 4.5: Efficiency of the SUPER_PHOTON70 trigger as a function of the $p_{T}$ of the photon. For $p_{T}^{\gamma}<$ $120 \mathrm{GeV}$ the efficiency is measured with electrons in $Z \rightarrow e e$ decays. For higher $p_{T}$ the efficiency is measured relative to JET_100 trigger samples.

\subsection{Background Subtraction}

The main background contributions to the prompt photon sample come from $\pi^{0}$ and $\eta$ decays at low $p_{T}$ and from cosmic muons at high $p_{T}$. Other minor background comes from electrons, 
whose showers in the calorimeter cannot be distinguished from those of the photons. Electrons from $Z$ and $W$ decays are removed from the sample by requiring the number of tracks pointing to the EM cluster to be 0 or 1 . One extra track is allowed to account for underlying event and pile-up energy around the cluster. In the case there is one track pointing to the photon cluster, this track is required to be soft compared to the measured energy of the photon (see cuts in Table 4.3). A cut on the missing $E_{T}$ further reduces the number of electrons coming from $W$ decays. We estimate a residual $1 \%$ of electrons in the first two bins from other measurements [48].

\subsubsection{Non-collision background}

Cosmic muons are the most important source of background for $p_{T}>100 \mathrm{GeV} / \mathrm{c}$. The cosmics rays interact with the CDF detector and produce photons through bremsstrahlung. These muons come from any direction, and they may not leave a track in the COT. The radiated photons are not related to the rest of the event, and therefore they will appear isolated in the calorimeter. Other non-collision backgrounds are due to beam-halo and PMT-spikes. The latter are caused by the overlap of soft physics events with large noise (spike) in one of the calorimeter PMTs. When only spikes are present, these are killed by the spike killer in the readout electronics, which removes events with zero $E_{P M T 1} \times E_{P M T 2}$, where 1 and 2 are the PMTs that read the tower (see Chapter 3). In the case soft physics events are detected by the PMTs, then both PMT1 and PMT2 will give a zero non-signal just as real events do. Beam-halos are proton (anti-proton) beams which are not coalesced and hit the beam pipe producing muons (together with many short-lived particles). Some of these muons may interact with the material of the CDF calorimeter producing photons.

All these processes contribute to the total $E_{T}$ of the event, giving rise to large missing $E_{T}$ $(M E T)$, and can be suppressed by applying a cut on this variable. For cosmics, beam-halo and PMT-spike suppression we require $M E T / E_{T}^{\gamma}<0.8$. This cut was obtained by comparing the observed MET distributions $v s$ the $p_{T}$ of the photon for all the measured $p_{T}$-range; an example is shown in Fig. 4.6. The remaining fraction of non-collision background in the sample was estimated using the EMTiming system described in Section 3.2.3.5.

The EMTiming detector measures the time at which the signal is detected in the calorimeter. The electronic gate opens about $20 \mathrm{~ns}$ before the collision is produced and closes at about $110 \mathrm{~ns}$ after. Fig. 4.7 shows an example of the EMTiming distribution for beam halos and cosmics. The collision is produced at $0.0 \mathrm{~ns}$. Photons from the hard scattering tend to populate the region between 30 to $90 \mathrm{~ns}$. Since halo photons are produced before the collision, they contribute to the 


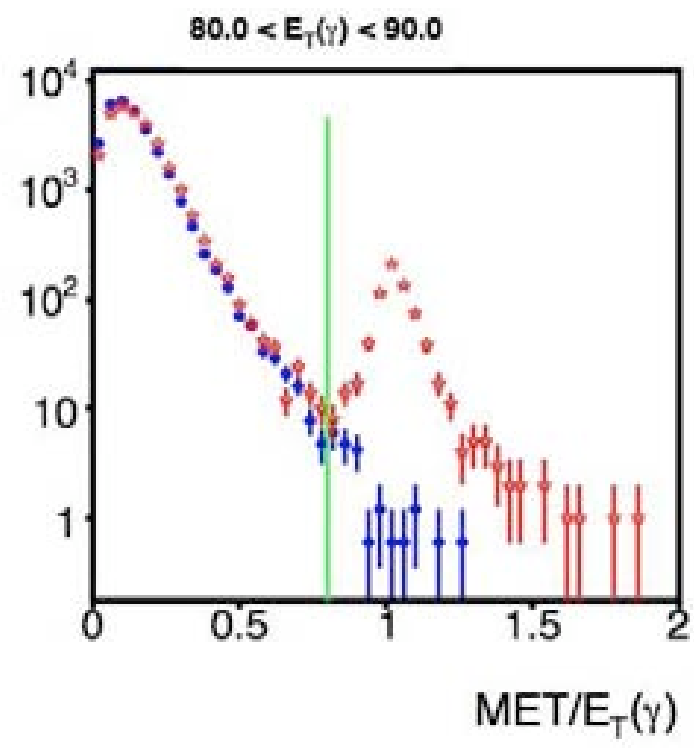

Figure 4.6: $M E T / E_{T}^{\gamma}$ distribution for the inclusive photon sample (in red) and a Monte Carlo inclusive photon sample (in blue) for $80<p_{T}^{\gamma}<90 \mathrm{GeV} / \mathrm{c}$. The vertical green line indicates the separation between $M E T / E_{T}^{\gamma}<0.8$ and $M E T / E_{T}^{\gamma}>0.8$.

peak in the negative region. The cosmic rays come randomly and arrive at the CDF calorimeter with a time uncorrelated to the collision, and their timing distribution is flat and dominates the region for timing above $20 \mathrm{~ns}$.

The EMTiming distribution was studied after the $M E T / E_{T}^{\gamma}$ cut was applied. The remaining events in the flat region and in the negative peak were used as an estimator of the remaining comic and halo photons for every bin in the photon $p_{T}$. We find the contribution from these photons is reduced to less than $1 \%$, so we consider it negligible. We estimated the efficiency of $M E T / E_{T}^{\gamma}$ cut on the signal photons using the same EMTiming distribution in a $Z \rightarrow e^{+} e^{-}$sample, and found it to be above $98.5 \%$.

\subsubsection{Light meson background}

At low $p_{T}$ the most important source of background comes from light mesons decays to two photons. These photons are in part eliminated with the isolation cuts at trigger and offline levels, and with the CES $\chi^{2}$ and the 2nd CES cluster cuts. However, when these photons are collinear, they fake a single photon shower in the calorimeter, reducing the effectiveness of the CES based cuts. 


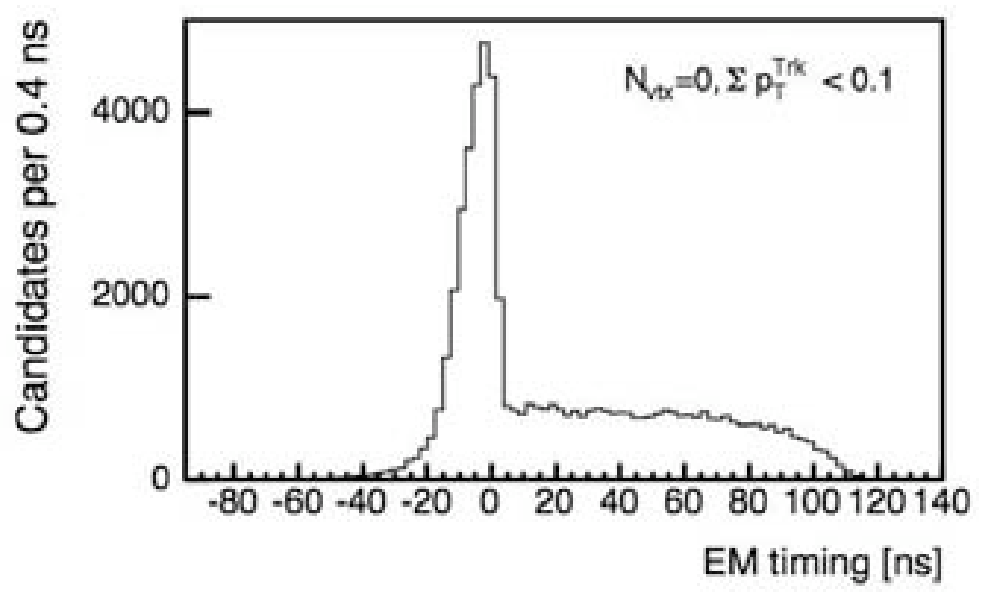

Figure 4.7: EMTiming distribution for beam halo and cosmic photons. The flat region is dominated by cosmic rays while the peak in the negative region is dominated by beam halos. There are turn-ons at $\sim-$ $20 \mathrm{~ns}$ and fall-offs at $\sim 110 \mathrm{~ns}$ because the electronics gate opens about $20 \mathrm{~ns}$ before the collision and lasts for about $110 \mathrm{~ns}$ after it.

Since in most of the cases photons from mesons will be accompanied by other hadronic particles that will leave part of their energy around the photon candidate, the isolation cut provides a highly effective way to remove these contributions. In the case the hadronization of the hard scattered parton results in most of the energy transferred to the mother meson, the resulting photons will be not eliminated by the photon ID cuts. These photons and cannot be distinguished from prompt photons on an event by event basis. Instead, this background is removed in a statistical manner.

In this measurement, the background subtraction method is based on the isolation energy in the calorimeter around the photon candidate. The fraction of the data attributed to prompt photon production (signal fraction) is estimated by fitting the isolation distribution in the data to signal and background Monte Carlo isolation templates for every bin in photon $p_{T}$. The signal template is constructed with photon Monte Carlo and describes the peak of the isolation in the data. The background template is developed from photons coming from mesons in a dijet Monte Carlo sample, and reproduces the high isolation tail in the data (see Fig. 4.8). 


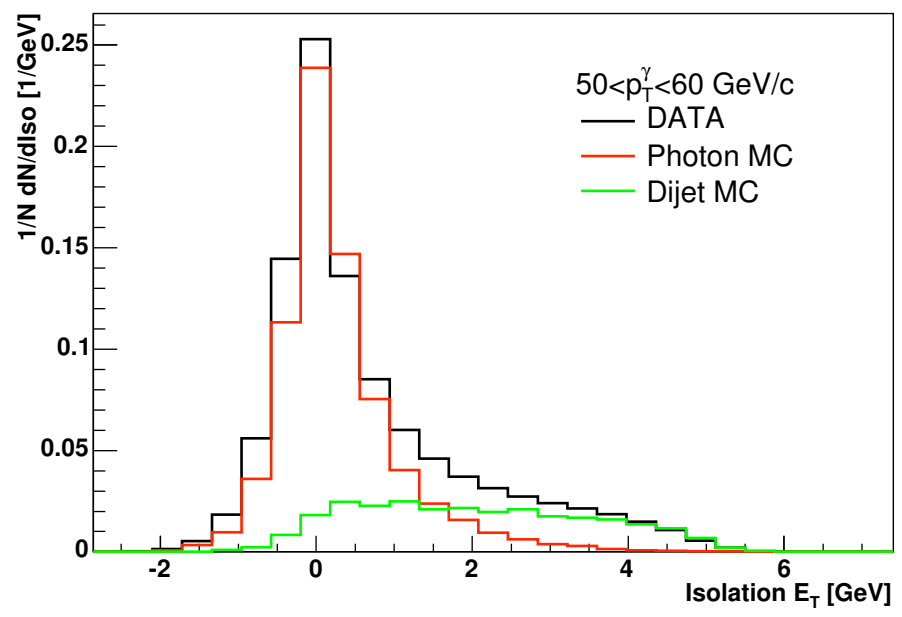

Figure 4.8: Calorimeter isolation distribution for data, photon Monte Carlo and dijet Monte Carlo for $50<p_{T}^{\gamma}<60 \mathrm{GeV} / \mathrm{c}$. The distributions are normalized to their area, and the photon and dijet shapes are weighted according to the predicted signal fraction for this $p_{T}$ bin.

\subsubsection{Isolation templates}

The signal template is constructed from PYTHIA photon Monte Carlo. We count both LO Matrix Element photons and photons from the parton shower. The ME photons will populate the peak of isolation, just as the direct photons in the PQCD calculations, while the photons produced promptly in the parton shower will be non-isolated for most of the cases, as occurs with the fragmentation component. Alternatively, one could consider using electrons for the construction of the signal templates. However, we do not use the isolation distribution from the $Z$ data sample as a signal template because it does not account for the two different components (direct + fragmentation) in the theory the same way the photon Monte Carlo does, and, moreover, it does not provide enough statistics above $70 \mathrm{GeV}$. Therefore, we take the approach of using photon Monte Carlo for the primary result, and use the electrons from $Z$ decays to set the systematics at low $p_{T}$.

To develop the templates for jets faking photons, we run our signal selection on inclusive dijet Monte Carlo samples (see Section 4.2). The templates are formed only by jets faking photons through mesons. We remove events where the reconstructed photon comes from a quark line (bremsstrahlung) since in the NLO calculations these photons constitute precisely the fragmentation component. The resulting template is flat in isolation as expected and is sculpted by 
the isolation trigger requirement $\left(E_{T}^{i s o} / E_{T}<0.10\right)$ for $p_{T}<90 \mathrm{GeV} / \mathrm{c}$. In the case the fake-photon is isolated, it is due to a fluctuation in the energy left to the meson during the hadronization of the hard scattered quark, so there is no a priori physical mechanism that would privilege the production of isolated photons from the production of non-isolated photons.

\subsubsection{The signal template correction}

The isolation distribution in the calorimeter is shown in Fig. 4.8 for $50<p_{T}^{\gamma}<60 \mathrm{GeV} / \mathrm{c}$. The peak in the data is described by the photon Monte Carlo, while the high isolation tail mostly comes from photons produced in meson decays, obtained with dijet Monte Carlo samples. At $p_{T}$ above $60 \mathrm{GeV}$, the signal template peak is shifted from the peak observed in the data for the same photon energies. At very high $p_{T}$, the peak in the data is not reproduced by the peak in the photon Monte Carlo template, which appears to be wider and whose center is displaced to higher isolation values.

We have investigated the possible sources of this discrepancy. The isolation in the photon Monte Carlo comes mainly from energy from the underlying event, the pile-up and the lateral shower leakage in the calorimeter (Section 4.3). In order to disentangle between these different effects, we studied the isolation in the calorimeter in four different regions of the space defined by the CES $x$ position of the shower maximum and the number of vertexes in the event $(x, n)$. In this space, we can remove the pile-up contributions by requiring no extra-vertices in the event. The leakage effects can also be minimized if we look only in the low $|x|$ region, or in the contrary, they can be maximized by looking in a large CES $x$ region. By requiring no extra-vertices and small CES $x$, we can study the underlying event effect without contamination from pile-up or lateral shower leakage. We also have studied the underlying event in the photon Monte Carlo sample using random cones in the region away from the photon and the back-to-back jet.

The results of these various studies are summarized in Appendix C. We find that the observed differences are not explained in terms of underlying event or the leakage and pile-up corrections applied to the measured isolation in the calorimeter. Thus, we conclude they are likely due to a combination of these effects together with other soft radiation effects and possibly to the simulation itself.

For the fitting procedure, we correct the shape of the peak in the photon Monte Carlo sample using the peak position and width from the data. We use photon data because this is the only available photon sample at high $p_{T}$. The adjustment consists of aligning the data and the template 
peaks and correct for the width of the peak in the template according to the observed width in the data. The signal fraction depends mostly on the relative fraction of events belonging to the peak or to the tail of the isolation distribution in the data, which our correction is not modifying. The dependence of the signal fraction on the details of the shape of the peak in the isolation distribution is very small and therefore, the correction has little effect on the signal fractions. In order to prove that, we performed a detailed and extensive study of the systematic uncertainty due the signal fraction, presented in the next Chapter. In any case, we find the effect of the correction of the templates in the signal fraction is less than $3 \%$ for all the measured range.

The correction is only applied to the photon Monte Carlo templates while dijet templates are left without any correction. The main reason for this choice is that the adjustment is derived exclusively using the peak in the data. Since the dijet templates are flat in isolation, there is no reason to assume the same correction would be valid here. On the other hand, the effect of the correction in the dijet templates is very small and is considered a as systematic of the measurement (see Chapter 5).

The isolation correction has the form of a $p_{T}$-dependent function. Its derivation is performed in four steps and the different terms of the correction are obtained by fitting the peaks in the data and the signal templates to a Gaussian. The different terms are explained below:

\section{- Align the data and template peaks}

The Monte Carlo template is displaced so the peak position in the Monte Carlo matches that in the data. This term is given by $f_{\text {shift }}\left(p_{T}\right)$ in eq. 4.1 and its functional form is shown in Fig. 4.9. The different points of the curve in Fig. 4.9 come from the difference between the means of the Gaussians in data and Monte Carlo.

\section{- Center the template at zero isolation}

The signal template is centered at zero isolation in order to properly reweight the width of the peak. This corresponds to $f_{\text {offset }}\left(p_{T}\right)$ in eq. 4.1. The function is displayed in Fig. 4.10 and is obtained by fitting the means of the Gaussians in the data distributions.

\section{- Weight the Monte Carlo peak width}

The ratios of the sigmas of the Gaussians obtained in data and Monte Carlo for every photon $p_{T}$ are fitted to $f_{\text {weight }}\left(p_{T}\right)$ in eq. 4.1. The fit result is displayed in Fig. 4.11.

\section{- Center the signal template back on its position}

After the peak width is corrected for, the signal template is centered again on the position given by $f_{\text {shift }}\left(p_{T}\right)$. 
The final isolation is given by:

$$
E_{T}^{i s o}\left(p_{T}\right)=\left(E_{T_{u} n c o r r}^{i s o}\left(p_{T}\right)-f_{\text {shift }}\left(p_{T}\right)-f_{\text {offset }}\left(p_{T}\right)\right) \times f_{\text {weight }}\left(p_{T}\right)+f_{\text {offset }}\left(p_{T}\right)
$$

where $E_{T_{u} \text { ncorr }}^{\text {iso }}\left(p_{T}\right)$ is the isolation as it comes out from the photon Monte Carlo. The three $p_{T^{-}}$ dependent terms of the correction derived from the fits are shown in Fig. 4.9, 4.10 and 4.11.

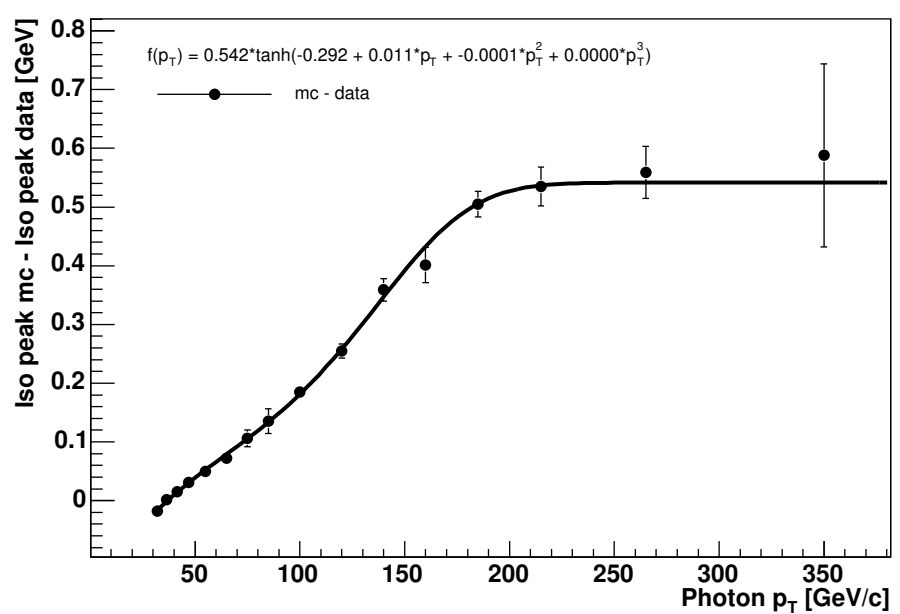

Figure 4.9: $f_{\text {shift }}\left(p_{T}\right)=0.542 \cdot \tanh \left(-0.292+0.011 p_{T}-9.4 \cdot 10^{-5} p_{T}^{2}+5.1 \cdot 10^{-7} p_{T}^{3}\right)$ function that aligns the data and template peaks.

\subsubsection{Fit results}

The primary fit is displayed in Fig. 4.13-4.18. The fit results are also shown in logarithmic scale. The $\chi^{2}$ of the fits is computed taking into account the statistical uncertainties in the data and in the Monte Carlo templates, and no systematics are included in the fits. The systematic uncertainties are discussed in the next Chapter.

For the final number of prompt photons, we integrate the signal template below $2 \mathrm{GeV}$. The final signal fraction together with the systematic uncertainties as a function of the photon $p_{T}$ is shown in Fig. 4.12 and Table 4.5. The fraction increases from $70 \%$ to about $98 \%$ as the photon $p_{T}$ increases. 


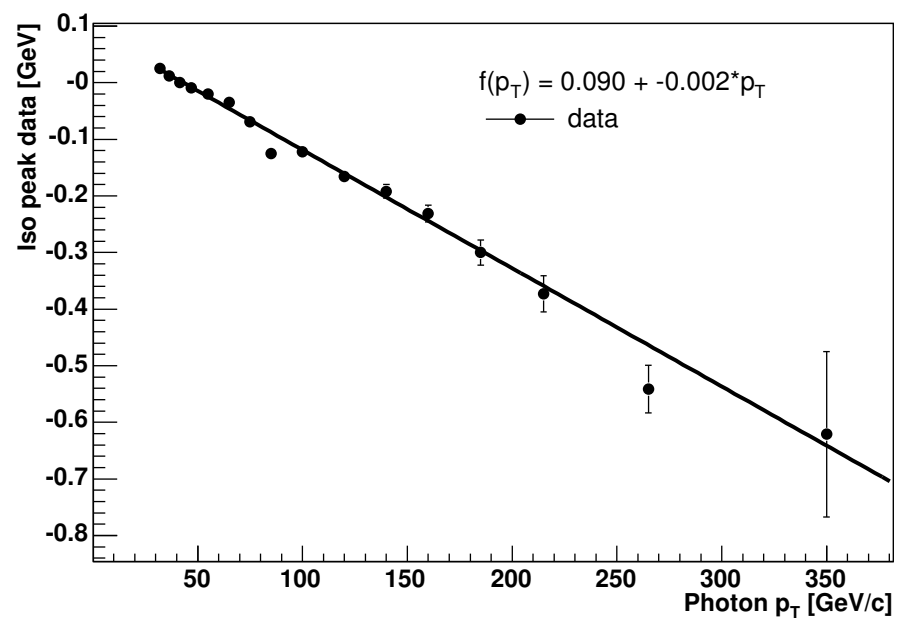

Figure 4.10: $f_{o f f s e t}\left(p_{T}\right)=0.09-0.002 p_{T}$ function that centers the template at 0 before being reweighted.

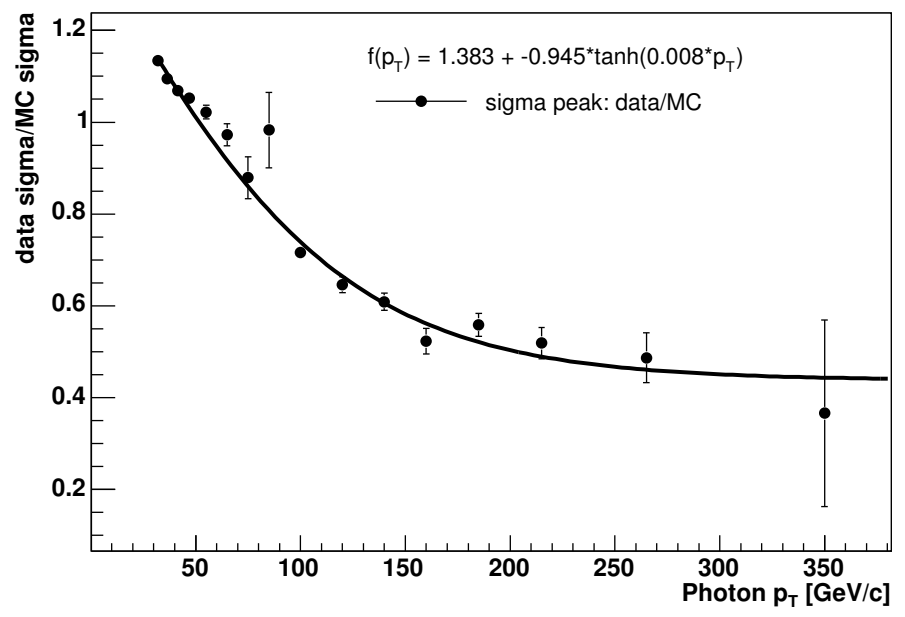

Figure 4.11: $f_{\text {weight }}\left(p_{T}\right)=1.383-0.945 \cdot \tanh \left(8.32 \cdot 10^{-3} p_{T}\right)$ function that weights the peak width in the Monte Carlo template. 


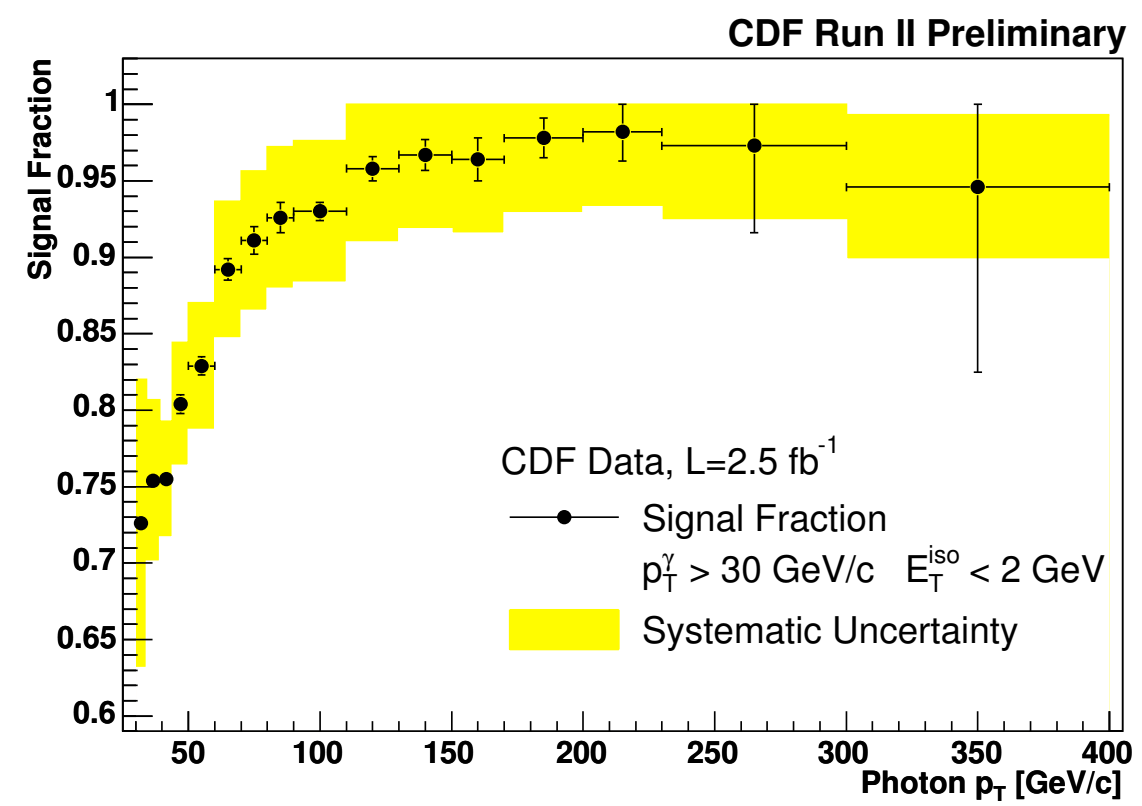

Figure 4.12: Signal fractions as a function of $p_{T}$ of the photon. The error bars associated to the points correspond to the statistical uncertainty in the fit, which comes from both the data and the Monte Carlo templates. The yellow band indicates the systematic uncertainty, described in detail in Chapter 5 . 

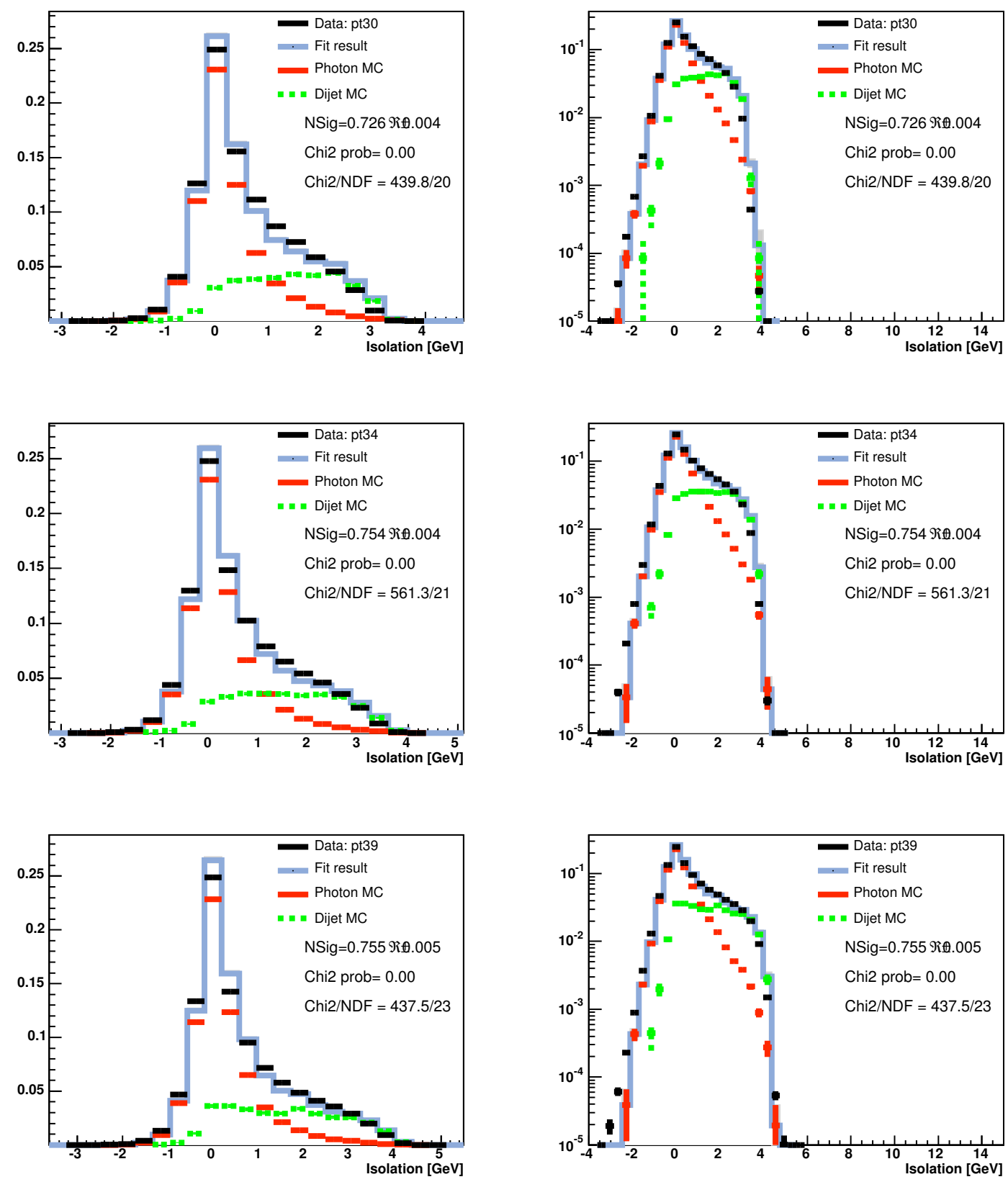

Figure 4.13: Fits to the isolation distribution in bins of $p_{T}$ for $30<p_{T}<44 \mathrm{GeV}$. Left column shows the fit results in linear scale. Logarithmic scales are displayed in right column. The signal template is from inclusive photon Monte Carlo, and the background template from dijet Monte Carlo with brem photons removed. 

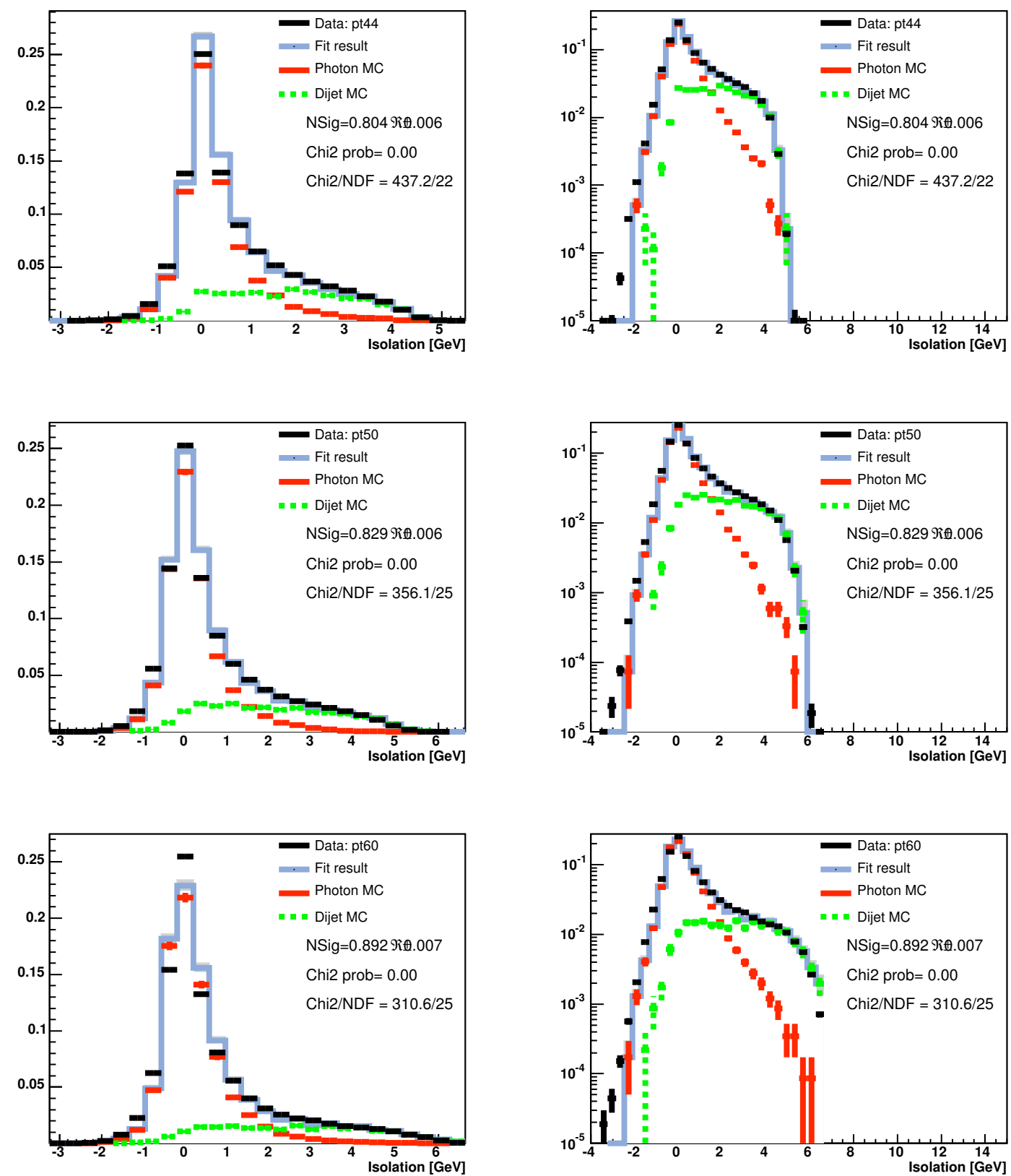

Figure 4.14: Fits to the isolation distribution in bins of $p_{T}$ for $44<p_{T}<70 \mathrm{GeV}$. Left column shows the fit results in linear scale. Logarithmic scales are displayed in right column. The signal template is from inclusive photon Monte Carlo, and the background template from dijet Monte Carlo with brem photons removed. 

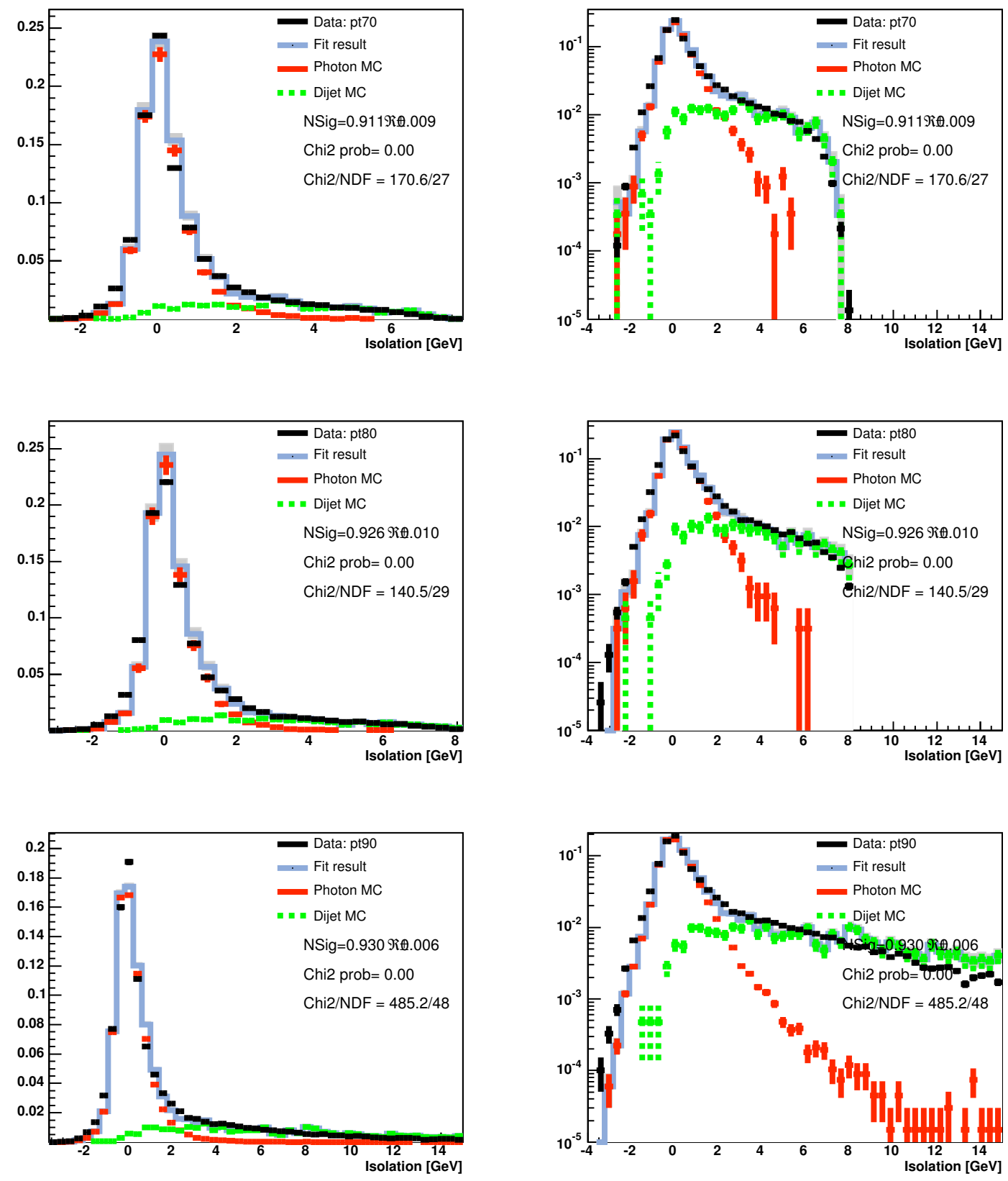

Figure 4.15: Fits to the isolation distribution in bins of $p_{T}$ for $70<p_{T}<110 \mathrm{GeV}$. Left column shows the fit results in linear scale. Logarithmic scales are displayed in right column. The signal template is from inclusive photon Monte Carlo, and the background template from dijet Monte Carlo with brem photons removed. 

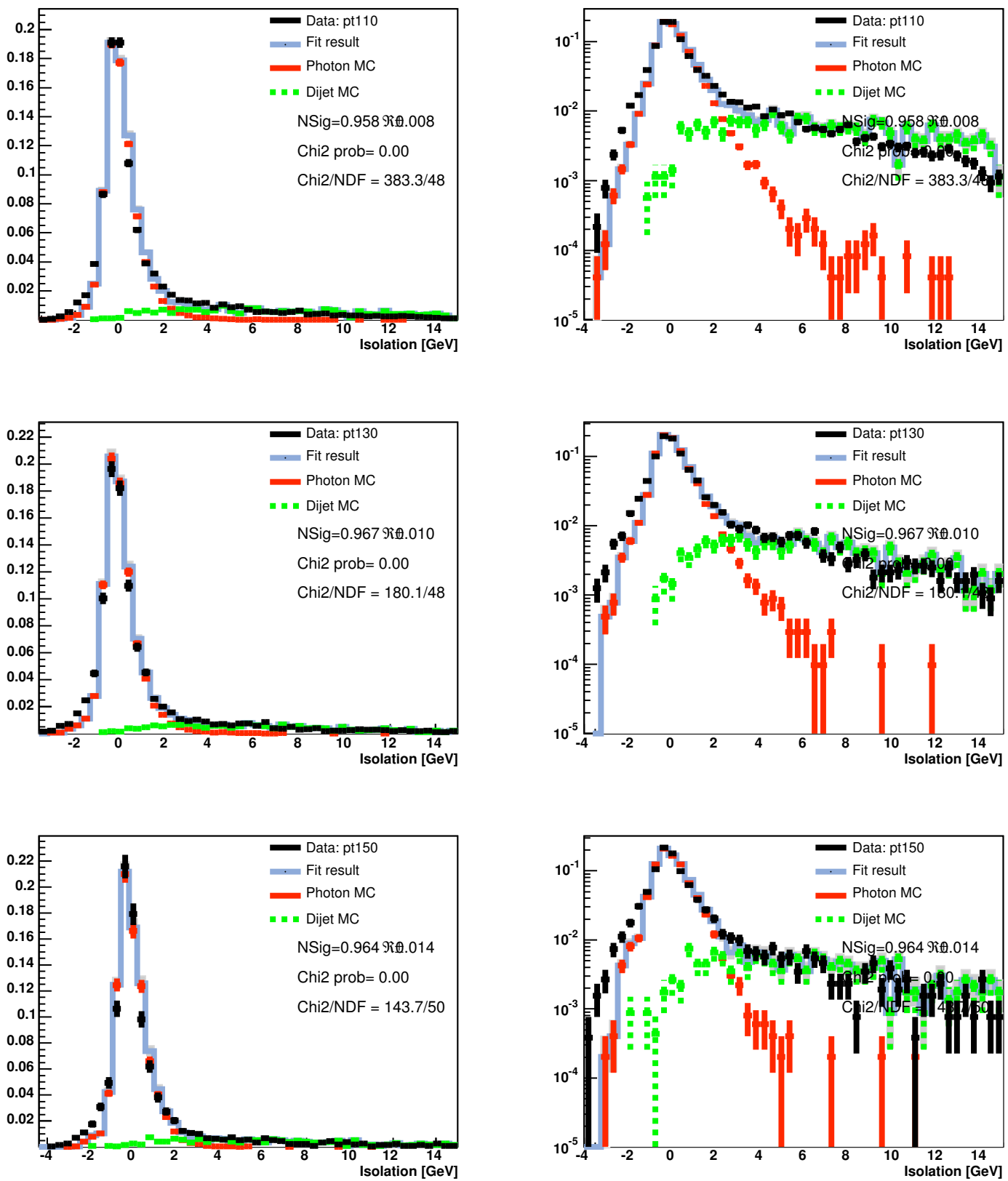

Figure 4.16: Fits to the isolation distribution in bins of $p_{T}$ for $110<p_{T}<170 \mathrm{GeV}$. Left column shows the fit results in linear scale. Logarithmic scales are displayed in right column. The signal template is from inclusive photon Monte Carlo, and the background template from dijet Monte Carlo with brem photons removed. 

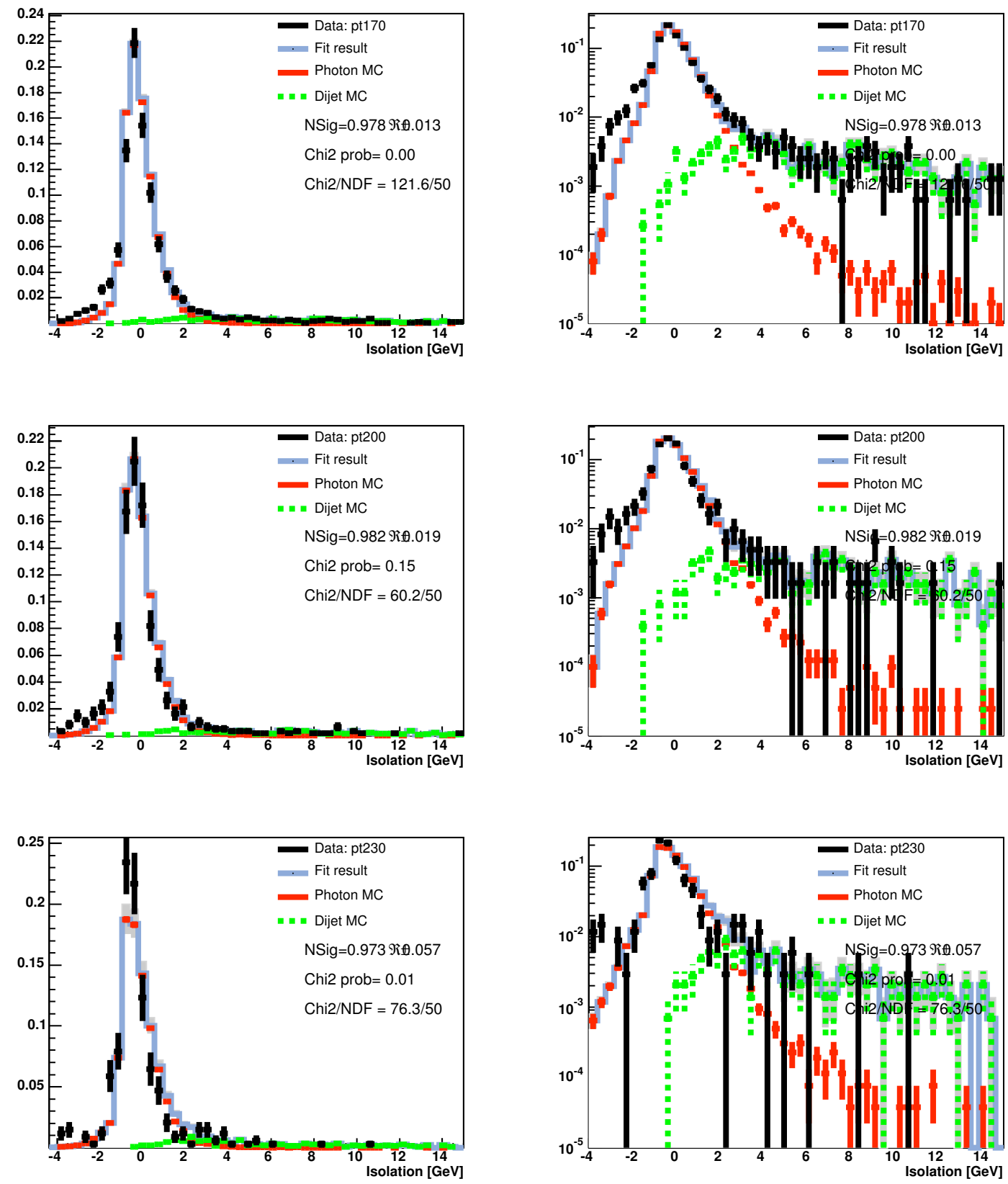

Figure 4.17: Fits to the isolation distribution in bins of $p_{T}$ for $170<p_{T}<300 \mathrm{GeV}$. Left column shows the fit results in linear scale. Logarithmic scales are displayed in right column. The signal template is from inclusive photon Monte Carlo, and the background template from dijet Monte Carlo with brem photons removed. 

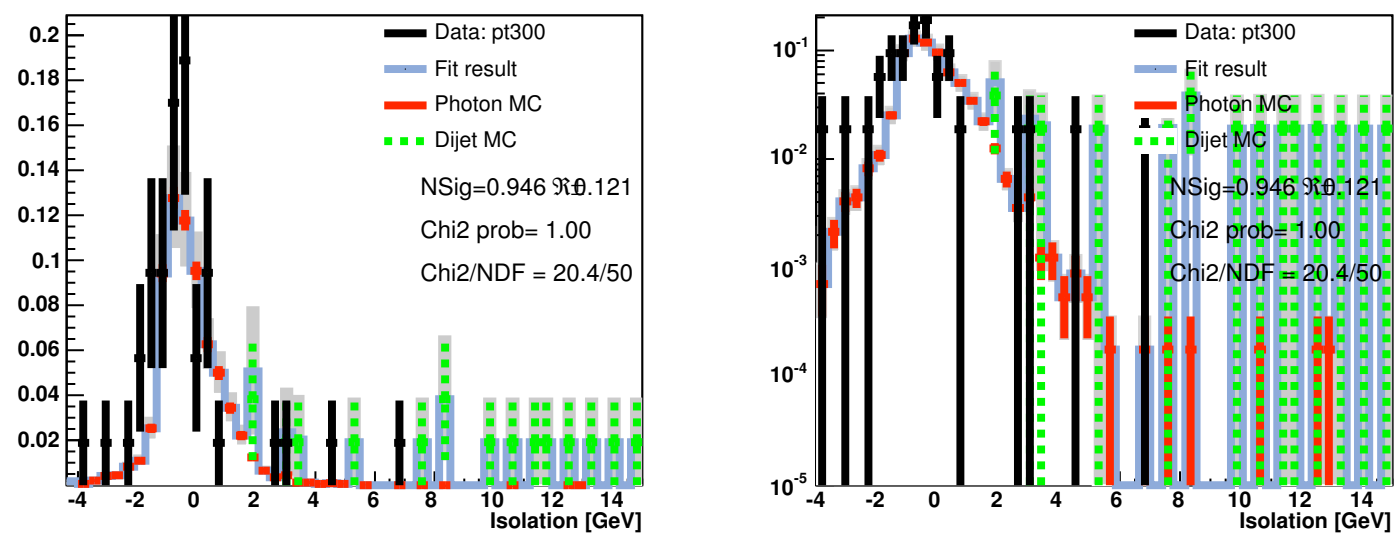

Figure 4.18: Fits to the isolation distribution in bins of $p_{T}$ for $300<p_{T}<400 \mathrm{GeV}$. Left column shows the fit results in linear scale. Logarithmic scales are displayed in right column. The signal template is from inclusive photon Monte Carlo, and the background template from dijet Monte Carlo with brem photons removed.

\subsection{Unfolding Factors}

The measured photon $p_{T}$ spectrum shape depends on the efficiency of the photon identification cuts, and on the detector acceptance and resolution. These are all dependences that must be removed from our measurement in order to compare the result to the theoretical predictions. This procedure is called "unfolding" of the cross section. In this measurement, we use the PYTHIA photon Monte Carlo sample to unfold the detector effects. The unfolding factors are computed bin by bin in $p_{T}$, and are defined as follows:

$$
U=\frac{\gamma_{\text {rec }} \text { passing ID cuts in Table } 4.3}{\gamma_{\text {gen }}\left(|\eta|<1.0, E_{T}^{i s o}<2 \mathrm{GeV}, p_{T}>30 \mathrm{GeV} / \mathrm{c}\right)}
$$

Here, the energy of the reconstructed photons has been corrected as explained in Section 4.4. The kinematic cuts for the photons at generator level are $p_{T}<30 \mathrm{GeV} / \mathrm{c},|\eta|<1.0$ and $E_{T}^{i s o}<2 \mathrm{GeV}$. The unfolding factors correct for acceptance and resolution effects, account for the efficiency of the photon selection in the calorimeter, and take care of the energy dependence of the energy scale correction, which was derived for energies around $50 \mathrm{GeV}$.

The correction due to the efficiency of the photon selection cuts is included in the unfolding factors. In order to evaluate the difference in the efficiency in data and Monte Carlo, electrons 


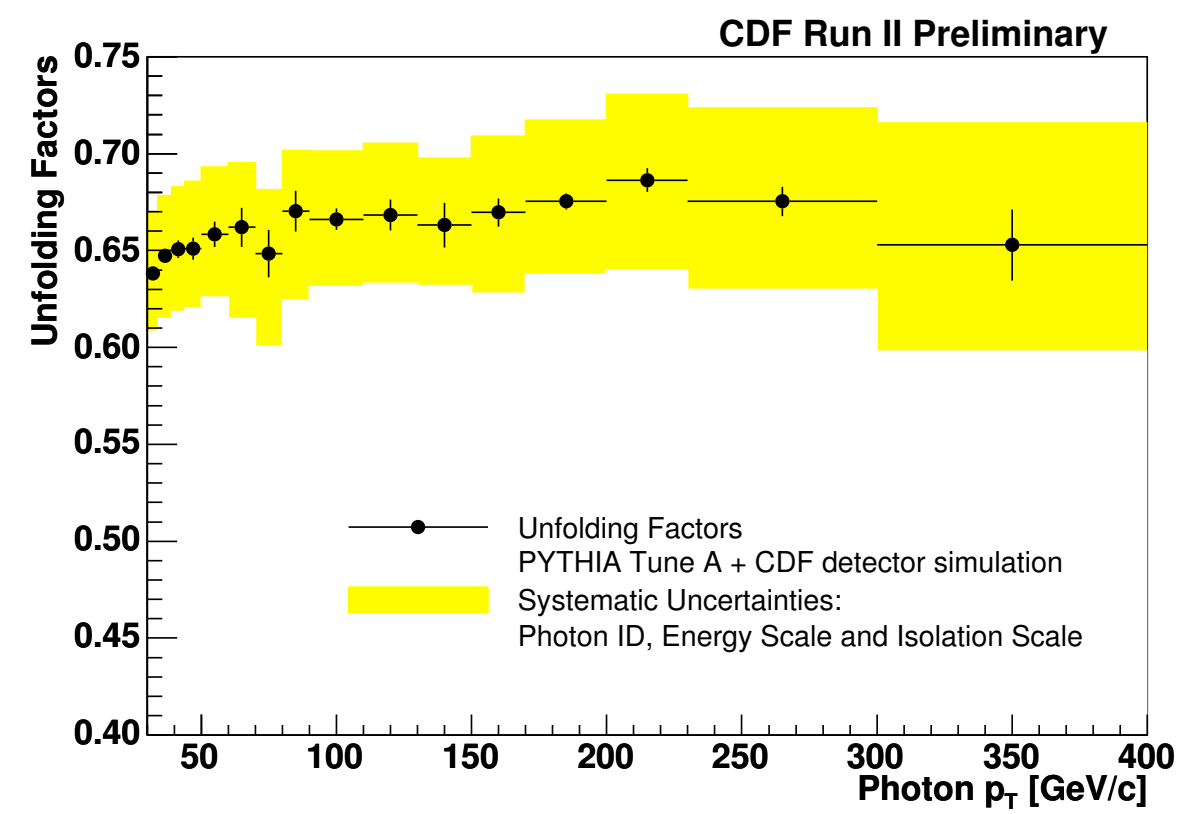

Figure 4.19: Unfolding factors as a function of the $p_{T}$ of the photon. The yellow band includes the systematic uncertainties due to the photon ID (acceptance + CES $\chi^{2}$ ) and the uncertainties due to the isolation and the energy scales (see next Chapter for details).

from $Z \rightarrow e^{+} e^{-}$decays in data and in simulated samples were used to verify that the Monte Carlo correctly reproduces the efficiencies of the photon ID cuts. The efficiencies predicted by the Monte Carlo samples were $1 \%$ higher than the ones predicted by electrons in data, factor that is included as a correction in our unfolding factors. The unfolding factors $v s$ the $p_{T}$ of the photon are shown in Fig. 4.19 and in Table 4.5. They vary between $64 \%$ and $69 \%$ in the whole measured $p_{T}$ range and do not have strong $p_{T}$ dependence. 


\begin{tabular}{c|ccc}
\hline \hline$p_{T}^{\gamma}[\mathrm{GeV} / \mathrm{c}]$ & $\varepsilon_{T}$ & $\mathcal{F} \pm$ sys $(\%)$ & $U \pm$ stat $(\%)$ \\
\hline $30-34$ & 0.989 & $0.726_{-0.130}^{+0.130}$ & $0.638 \pm 0.003$ \\
$34-39$ & 0.995 & $0.754_{-0.070}^{+0.070}$ & $0.648 \pm 0.003$ \\
$39-44$ & 0.998 & $0.755_{-0.050}^{+0.050}$ & $0.651 \pm 0.005$ \\
$44-50$ & 0.998 & $0.804_{-0.050}^{+0.050}$ & $0.651 \pm 0.006$ \\
$50-60$ & 0.999 & $0.829_{-0.050}^{+0.050}$ & $0.658 \pm 0.007$ \\
$60-70$ & 1.000 & $0.892_{-0.050}^{+0.050}$ & $0.662 \pm 0.010$ \\
$70-80$ & 1.000 & $0.911_{-0.050}^{+0.050}$ & $0.648 \pm 0.013$ \\
$80-90$ & 1.000 & $0.926_{-0.050}^{+0.050}$ & $0.670 \pm 0.011$ \\
$90-110$ & 1.000 & $0.930_{-0.050}^{+0.050}$ & $0.666 \pm 0.006$ \\
$110-130$ & 1.000 & $0.956_{-0.050}^{+0.042}$ & $0.668 \pm 0.009$ \\
$130-150$ & 1.000 & $0.967_{-0.050}^{+0.033}$ & $0.663 \pm 0.012$ \\
$150-170$ & 1.000 & $0.964_{-0.050}^{+0.036}$ & $0.670 \pm 0.008$ \\
$170-200$ & 1.000 & $0.978_{-0.050}^{+0.022}$ & $0.675 \pm 0.005$ \\
$200-230$ & 1.000 & $0.982_{-0.050}^{+0.018}$ & $0.686 \pm 0.007$ \\
$230-300$ & 1.000 & $0.973_{-0.050}^{+0.027}$ & $0.675 \pm 0.008$ \\
$300-400$ & 1.000 & $0.946_{-0.050}^{+0.050}$ & $0.653 \pm 0.020$ \\
\hline \hline
\end{tabular}

Table 4.5: Trigger efficiencies $\left(\varepsilon_{T}\right)$, signal fractions $(\mathcal{F})$ together with the systematic uncertainties, and unfolding factors $(U)$ with the associated statistical uncertainties as a function of the photon transverse momentum. The trigger efficiencies correspond to the PHOTON_25_ISO trigger for $p_{T}<90 \mathrm{GeV} / \mathrm{c}$, while the SUPER_PHOTON70 efficiencies are those for $p_{T}>90 \mathrm{GeV} / \mathrm{c}$. In the trigger, the statistical uncertainties are smaller than $1 \%$ for all the measured range, while the systematic uncertainties are small and are neglected. All the systematic uncertainties are described in detail in Chapter 5. 



\section{Chapter 5}

\section{Systematic Uncertainties}

In this chapter we will provide a detailed description of the systematics uncertainties that affect the measurement. The most important sources of systematics come from the signal fraction at low $p_{T}$ and from the photon energy scale at high $p_{T}$. Smaller contributions to the systematic error are due to the isolation scale and to the photon ID efficiencies.

\subsection{Systematic in the Signal Fractions}

The signal fractions are one of the largest corrections at low $p_{T}$. We have studied the fitter results through a variety of methods, described below.

\subsubsection{Methods to estimate the systematic uncertainty}

- Electrons from $\mathbf{Z}$ decays in data:

We take advantage again of the similarity between the EM showers for photons and electrons and perform the fit using signal templates constructed with tight electrons from $Z$ 's in the lower $p_{T}$ bins until $70 \mathrm{GeV}$, where the $Z$ sample still provides sufficient statistics. The resulting fits are displayed in Appendix A (Fig. A.1 and A.2). 


\section{- CES/CPR methods:}

The CES and CPR methods [53] provide an estimate of the signal fraction which is completely independent from the nominal procedure. We use these methods in the first bins, where their systematic uncertainty is below $10 \%$. Moreover, the CES/CPR methods were calibrated with the standard photon identification in CDF, in which the CES $\chi^{2}<20$ cut is required throughout all the $p_{T}$ bins, while this analysis removes the CES $\chi^{2}$ cut for $p_{T}$ above $90 \mathrm{GeV}$.

The CES method estimates the prompt photon fraction by using the lateral shower profile of the photon candidates, measured with the CES detector. It can only be applied for $p_{T} \simeq 40 \mathrm{GeV} / \mathrm{c}$, since for higher $p_{T} \mathrm{~s}$ the shower profiles from prompt and meson photons cannot be distinguished anymore. The CPR method is based on the different conversion rate of photons $(\sim 60 \%)$ and background $(\sim 80 \%)$ in the material before the calorimeter, and can be applied to any $p_{T}$, though we use it as a cross-check only for $p_{T}>40 \mathrm{GeV} / \mathrm{c}$.

In the CES method, the efficiency of a CES $\chi^{2}<4$ cut (defined in Section 4.3.1) is estimated for Monte Carlo samples of pure photons $\left(\varepsilon_{\gamma}\right)$ and photons from mesons $\left(\varepsilon_{b}\right)$. If photon candidates in the data sample come from prompt photons and photons from meson decays, then:

$$
\varepsilon \cdot N=\varepsilon_{\gamma} \cdot N_{\gamma}+\varepsilon_{b} \cdot N_{b}
$$

where $N_{\gamma}$ is the number of photon candidates in the data and $\varepsilon$ is the efficiency of the CES $\chi^{2}<4$ cut in the data sample under study. From this expression and provided that $N=N_{\gamma}+N_{b}$, the number of prompt photons in the data is given by:

$$
N_{\gamma}=\left(\frac{\varepsilon-\varepsilon_{b}}{\varepsilon_{\gamma}-\varepsilon_{b}}\right) N
$$

The CPR method estimates the photon fraction by using the same formula in eq. 5.2. The efficiencies $\varepsilon_{\gamma}$ and $\varepsilon_{b}$ are defined as the fraction of photon candidates which produce a pulse height greater than that of a minimum ionizing particle $(500 \mathrm{fc})$ in the CPR within a 66 mrad window (5 CPR channels) around the photon direction, which gives an estimator of the conversion rate for these photons.

The CES and CPR methods have considerable systematic uncertainties which increase with the photon $p_{T}$. The photon fraction measured with the profile method is highly sensitive to slight differences between the electron and the photon showers. In addition, the showers used to identify the photon signals were measured under test beam conditions, which are 
different from those in the collider running. The background composition also contributes to the total uncertainty, of the order of $\sim 8.5 \%$ at $p_{T}^{\gamma}=40 \mathrm{GeV} / \mathrm{c}$.

The CPR conversion method presents two major uncertainties which also increase with the photon $p_{T}$. First, for low energy photons it is possible for a part of the EM shower to travel at a very large angles, almost backward, with respect to the incoming photons. These albedos or backscattered EM showers may convert and produce hits in the CPR and contribute to enhance $\varepsilon_{b}$, with a rate increasing with the energy of the photon. Due to this, in some cases the background hit rates must be corrected based on the signal fractions obtained from the isolation fitting procedure in the case of negative signal factions [53], resulting from situations in which $\varepsilon_{b}>\varepsilon_{\gamma}$.

\section{- 2-bin approach:}

In this method, we divide the isolation into two bins, above and below $2 \mathrm{GeV}$, for all photon $p_{T}$. The original isolation distributions have $0.4 \mathrm{GeV} / \mathrm{bin}$ division, while the 2 bin templates have one bin of $6 \mathrm{GeV}$ containing the peak of the distribution and another $11 \mathrm{GeV}$ bin for the tail. This removes all details of the shapes and just compares the relative yields in the high and the low isolation regions. Since it is not sensitive to the details of the isolation distribution shape, this method can be applied to both corrected and uncorrected signal templates and can be used to quantify the impact of the isolation correction in the photon purity.

\section{- Corrected dijet Monte Carlo templates:}

The nominal result uses corrected photon Monte Carlo templates while the dijet templates are left without any correction. The reason for this choice is that the isolation correction is based exclusively on the peak shapes in data and photon MC, and the dijet isolation shape is flat, so it is no obvious why the same correction would apply. On the other hand, as can be seen in the fit figures (Fig. 4.13 -4.18), the uncorrected dijet template describes well the high isolation tail in the data. However, since we could imagine that the dijet templates are affected by similar effects to those observed in the signal templates, we perform one more check and use corrected dijet templates for the fitting procedure. The fit results are shown in Appendix B (Fig. B.1 - B.6). 


\subsubsection{The systematic uncertainty}

The summary of the signal fractions obtained with all the different methods and templates is shown, together with the systematic band, in Fig. 5.1.

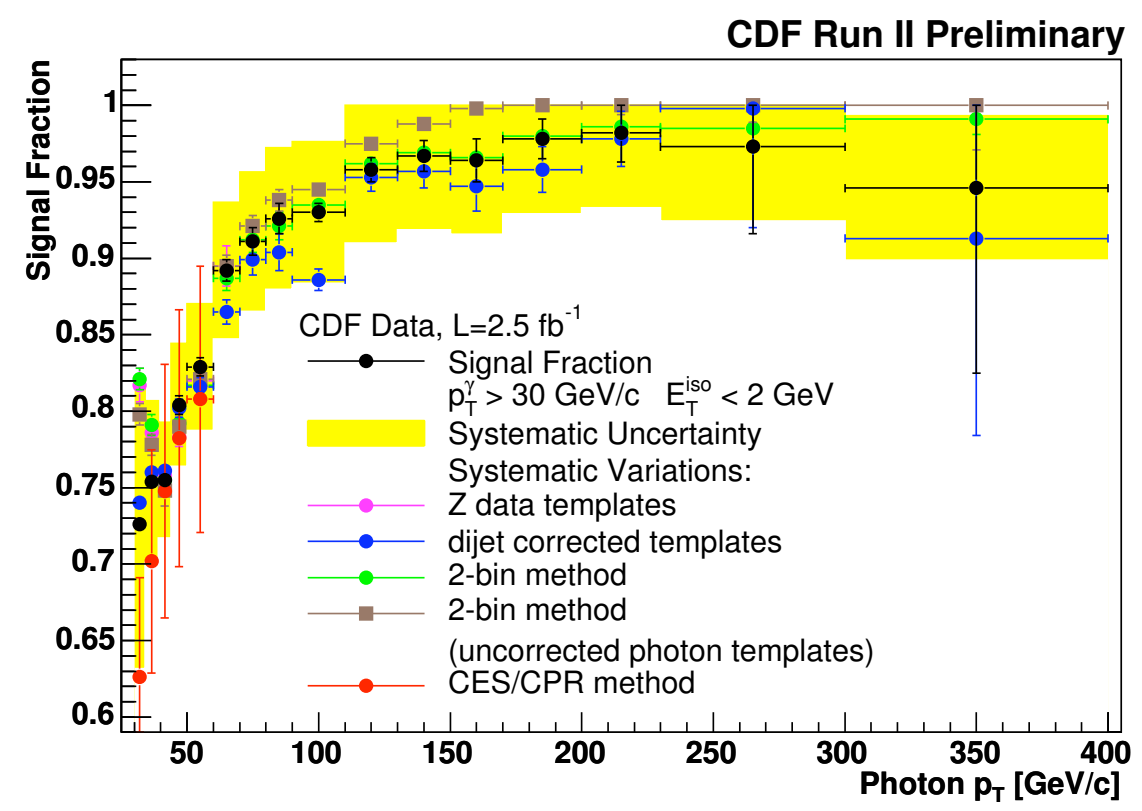

Figure 5.1: Summary of the signal fractions for all the different methods used to estimate the systematics together with the nominal result as a function of the photon $p_{T}$. The systematic uncertainty is indicated by the yellow band, and it is chosen to cover all the points.

The signal fractions in the two first bins from the $Z$ templates and the 2-bin systematics are above the signal fractions given by the photon Monte Carlo. For the third bin they both decrease to go closer to the nominal result, giving an unphysical behaviour (we expect the purity to monotonically increase with the photon $p_{T}$ ). In the Z's, the abnormal behaviour at low $p_{T}$ may be caused by the presence of residual background under the $Z$ peak, which would contribute to increase the signal fraction. To test this hypothesis, we use the photon and the dijet templates to fit the $Z$ templates in the two first bins. The fit results for the two first bins are presented in Fig. 5.2. In the first bin the fits estimate a 5\% residual background in the $Z$ template. In the second bin the residual background predicted by the fitter is $1.5 \%$. For the rest of the bins where the $Z$ results behave normally, the residual background predicted by the fitter is zero or compatible with 
zero within the statistical errors, which are of the order of $0.5 \%$. This partially accounts for the difference seen in the two first bins.
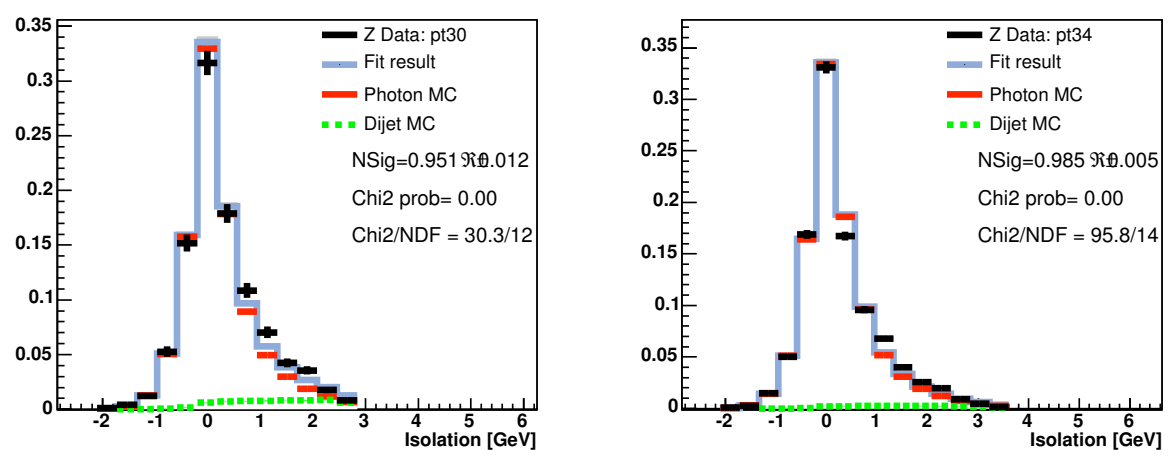

Figure 5.2: Fits of the $Z$ template for the two first bins $\left(30<p_{T}<34\right.$ and $\left.34<p_{T}<39 \mathrm{GeV} / \mathrm{c}\right)$. The $Z$ templates are fitted to the photon and dijet templates to estimate the residual background under the $Z$ mass peak.

In the case of the 2-bin method, the templates division at $2 \mathrm{GeV}$ isolation may be too far from the peak for the two first bins, where the tail is highly constrained and modulated by the trigger requirements. If this is the case, the $2 \mathrm{GeV}$ division is not able to resolve the peak from the tail in the data template (see for example the first plot of Fig. 4.13). To cross-check that, for these two bins, we separate the peak and tail components at $1 \mathrm{GeV}$ and compare the result to the signal fractions obtained from the fit for isolation $<1$. The result of the comparison is summarized in Table 5.1. With this, the nominal results given by the fitter and by the 2-bin method are different by a $3 \%$ at most.

\begin{tabular}{r|rrr}
\hline \hline$p_{T}^{\gamma}[\mathrm{GeV} / \mathrm{c}]$ & Fit result & 2-bin method & Fit result/2-bin method \\
\hline $30-34$ & $0.794 \pm 0.004$ & $0.820 \pm 0.006$ & 0.968 \\
$34-39$ & $0.811 \pm 0.004$ & $0.810 \pm 0.006$ & 1.001 \\
\hline \hline
\end{tabular}

Table 5.1: 2-bin method results compared to the fit result when the templates are divided at isolation of $1 \mathrm{GeV}$.

The 2-bin approach is not sensitive to the details of the isolation shape and therefore it can be used to evaluate the impact of the isolation template correction in the signal fractions. The method is applied to the uncorrected signal templates, and the resulting signal fractions are shown in Fig. 5.1 together with the results from the other methods. As can be seen from the figure, all the 
points are close to the nominal signal fractions, though at high $p_{T}$ all of them fall systematically above the nominal signal fractions, as a result of the observed trend in the peak of the uncorrected signal templates. Since the peak becomes wider as we go to higher energies, its contribution tends to dominate the higher isolation region, producing an enhancement of the signal fractions. Overall, the correction changes the signal fractions predicted by the 2-bin method by less than $3 \%$. Compared to the nominal results, the effect of the correction is within $5 \%$ for all the bins.

We have also studied the effect of the correction in the dijet templates. The resulting signal fractions are represented by the blue dots in Fig. 5.1. In most cases, the observed variation in the signal fractions due to the changes in the dijet template are covered by the statistical errors of the fit, which are of the order of few $\%$. As expected, the correction has little effect on the dijet template and on the fit results, supporting our choice of taking the non corrected dijet templates for the determination of the nominal signal fractions.

The CES/CPR signal fractions for the two first bins are below the photon Monte Carlo ones, but they agree within the systematic uncertainties of the CES/CPR methods (see Fig. 5.1), which have been discussed before in this Section. For the rest of the bins they come closer to the nominal signal fractions.

After studying the systematics with these different methods, we conclude that the fit results are very robust and we characterize an uncertainty of $13 \%$ (7\%) for the first (second) bin according to the variations shown by CES/CPR and the $Z$ templates. The uncertainty decreases to $5 \%$ at high $p_{T}$, covering the largest observed difference in the bins above $39 \mathrm{GeV} / \mathrm{c}$. The uncertainty from the signal fractions is the dominant one in the cross section in the low $p_{T}$ region.

\subsection{Systematics due to photon ID efficiency}

The uncertainty in the photon ID efficiency has two major contributors, the uncertainty due to the CES $\chi^{2}$ cut, and the photon acceptance uncertainty. The total photon ID uncertainty is shown in the total systematic uncertainty plot (Fig. 5.7) and in Tables 5.2 and 5.3.

\subsubsection{The CES $\chi^{2}$ cut efficiency}

The CES $\chi^{2}$ cut is only applied in the range where the PHOTON_25_ISO trigger is used, for $p_{T}<90 \mathrm{GeV} / \mathrm{c}$. Its efficiency is measured with the photon Monte Carlo and cross-checked with 
electrons from Z's in data; both are consistent and around 98\%, as shown in Fig. 5.3.

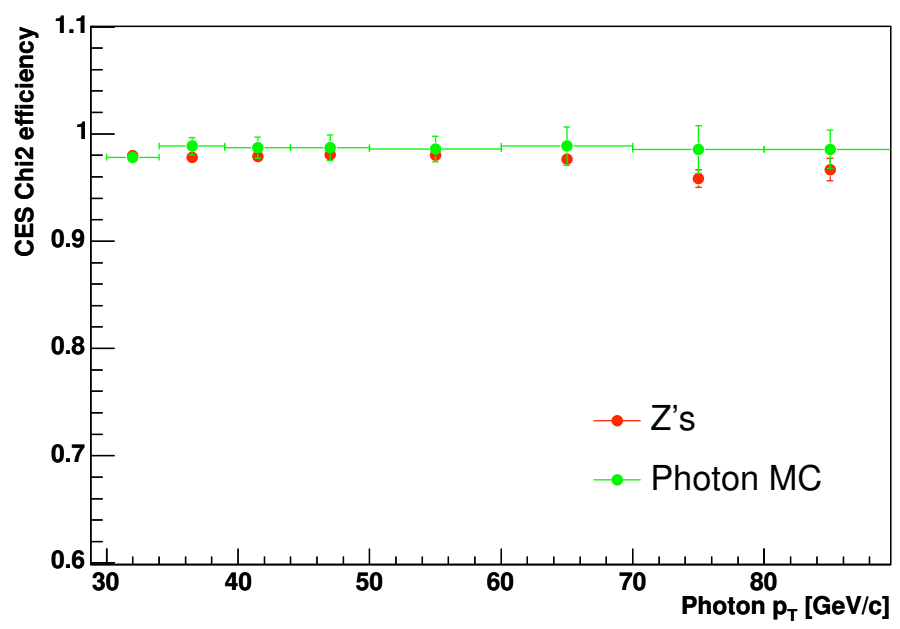

Figure 5.3: The CES $\chi^{2}<20$ cut efficiency predicted by the photon Monte Carlo and with electrons from $Z$ 's in data as a function of the $p_{T}$ of the photon.

For the last two bins in Fig. 5.3 the $Z$ points start to decrease compared to those obtained from the photon Monte Carlo. But the statistics of the $Z$ sample for the two last bins is poor, and we cannot conclude there is a real trend. On the other hand, previous measurements at CDF [44] have shown that the CES $\chi^{2}$ efficiency in data decreases at high $p_{T}$, while Monte Carlo predicts a flatter behaviour. To investigate if what we see in the two last bins of Fig. 5.3 are fluctuations or a real trend in the data, we use the photon data above $90 \mathrm{GeV}^{1}$. To measure the CES $\chi^{2}<20$ cut efficiency for our selection, we construct templates including this cut and count the corresponding event yields. The efficiency is given by the ratio:

$$
\varepsilon_{\chi^{2}}=\frac{\left(N_{\text {data }} \cdot \mathcal{F}\right)_{\chi^{2}}}{\left(N_{\text {data }} \cdot \mathcal{F}\right)_{N O \chi^{2}}}
$$

The result for the first bins together with the efficiencies predicted by the photon Monte Carlo and the electrons are displayed in Fig. 5.4. The 5\% yellow band is the assigned systematic uncertainty and covers the difference between the predictions of photons in the data and in the Monte Carlo samples.

\footnotetext{
${ }^{1}$ Below this energy the CES $\chi^{2}$ cut is already in the trigger requirements, so it is not possible to have a data photon sample for the efficiency measurement.
} 


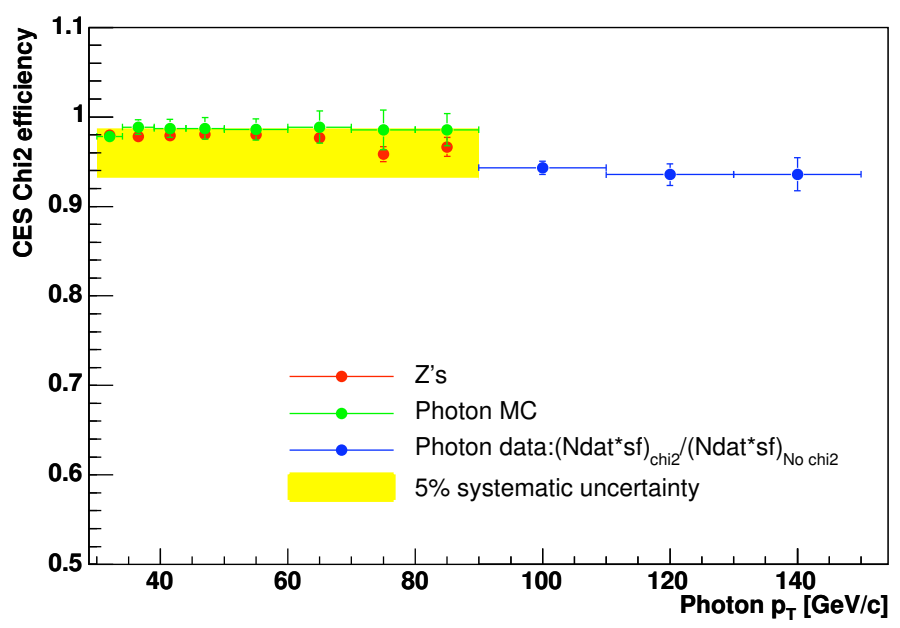

Figure 5.4: The systematic uncertainty due to the CES $\chi^{2}$ efficiency as a function of the photon $p_{T}$. The red points are the efficiencies as measured by electrons from $Z$ decays, the green dots are the efficiencies predicted by the photon Monte Carlo, and the blue dots are the efficiencies measured with the inclusive photon sample. The systematic uncertainty covers the difference between the different estimations and is indicated by the yellow band.

\subsubsection{Uncertainty in the photon acceptance}

A 3\% systematic uncertainty on the photon acceptance is included to cover the observed difference caused by the particular choice of the PDF in the PYTHIA Monte Carlo. The value is based on similar studies carried out in [44].

\subsection{Systematics due to the Photon Energy Scale}

We consider $\pm 1.5 \%$ systematics in the photon energy scale, estimated by comparing electrons in data and Monte Carlo samples. The energies of the electrons have been previously corrected as explained in Section 4.3. This correction was derived from electron data and Monte Carlo samples for energies $\sim 50 \mathrm{GeV}$ and applied as a correction factor to the photons and electrons without considering any energy or $\eta$ dependence. Therefore, we expect the energy correction will bring agreement between photons in data and Monte Carlo for energies around this value, but not 
for all the measured $E_{T}$ range $^{2}$. In the case of the $\eta$ position of the photon, we do not expect a large dependence, since all the possible values of $\eta$ are integrated in the cross section.

To quantify the uncertainty in the photon energy scale we use electron samples to investigate the dependence of the energy scale with the position and the energy of the photon in data and Monte Carlo samples. The maximum observed difference is taken as a systematic uncertainty. The energy scale is defined as the ratio between the invariant mass of the reconstructed electron pair in the calorimeter and the $Z$ mass as given by the PDG $\left(E_{\text {scale }}=M_{\text {rec }} / M_{P D G}\right)$. In Fig. 5.5 we plot the ratio between the scales for data and Monte Carlo $v s$ the sum of the energies of the two electrons from the $Z$ decay. From the figure, we see that the observed differences are covered by a $1.5 \%$ uncertainty. The effect of the photon energy scale uncertainty in the cross section is determined through the unfolding factors, using a photon Monte Carlo sample and by varying the energy of the reconstructed photon by $\pm 1.5 \%$ while the energy of the generated photon is kept untouched. The effect increases from $6 \%$ at low $p_{T}$ to $13 \%$ at high $p_{T}$, where it is the dominant source of systematics.

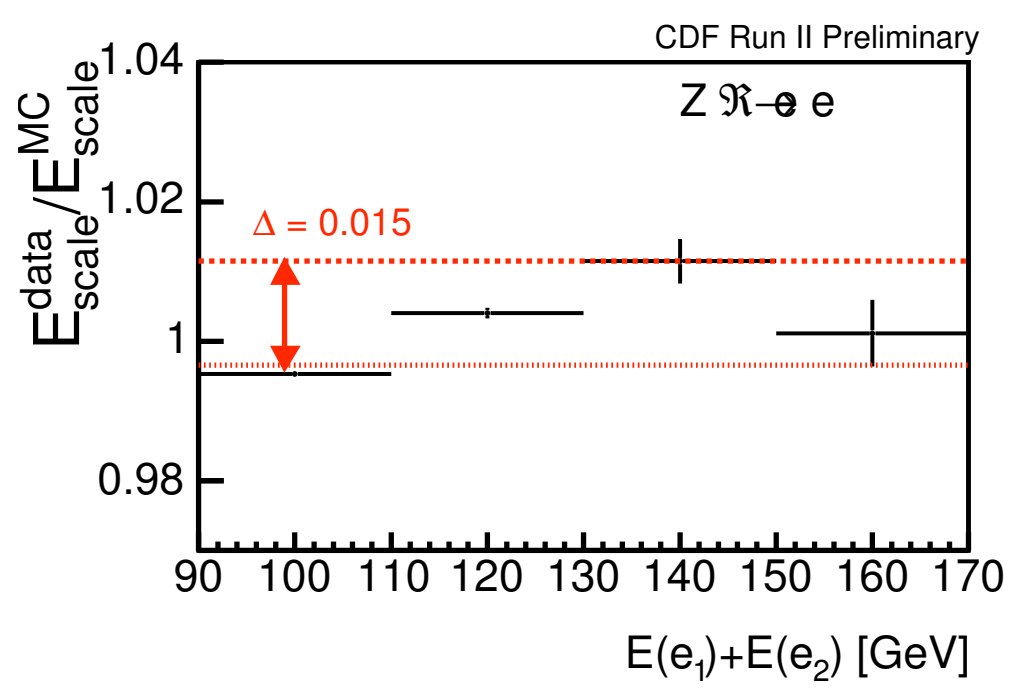

Figure 5.5: Ratio of the energy scale in data and Monte Carlo $Z$ samples as a function of the sum of the energies of the two electrons from the $Z$ decay.

\footnotetext{
${ }^{2}$ The correction for the dependence of the energy scale with the photon energy is done through the unfolding factors.
} 


\subsection{Systematics due to the Photon Isolation Scale}

Given that the method employed for the background estimation is based on the isolation shapes in the calorimeter, we include an uncertainty on the isolation scale to account for possible differences between the isolation in data and in Monte Carlo. The uncertainty in the photon isolation scale is $\pm 10 \%$ based on our knowledge of the energies of the order of few a GeV. The effect of the isolation scale uncertainty in the cross section is of $1 \%$ at low $p_{T}$ and decreases to $0.6 \%$ at high $p_{T}$, and is obtained through the unfolding factors by varying the cut in the reconstructed isolation to $2.2 \mathrm{GeV}$ and $1.8 \mathrm{GeV}$, while the cut at parton level is kept fixed at $2 \mathrm{GeV}$.

\subsection{Other sources of systematics}

We consider other sources of systematics in the measurement due to the amount of material of the detector and to the trigger efficiency, explained below. Uncertainties due to the reweighting of the photon Monte Carlo and to the collision background are negligible.

\section{- Uncertainty due to the amount of material}

In the path from the interaction point to the calorimeter, there are some photons that may interact with the tracking system, producing an $e^{+} e^{-}$pair. These photons are removed by our selection cuts, which reject any EM cluster with a high $p_{T}$ track pointing to it, and therefore they do not contribute to the cross section. The probability for a photon to convert depends on the amount of transversed material, and a proper simulation of the detector is crucial to correctly reproduce this effect in the Monte Carlo.

The CDF Electroweak Group [56] has estimated the uncertainty on the amount of tracking material $\left(\Delta_{\text {mat }}\right)$ in the simulation of the detector by comparing the fractions of electrons in the $E / p$ tail in $\mathrm{MC}$ and data, and found that $\Delta_{m a t}$ is $\approx 1 \%$. The conversion probability of photons is parametrized as

$$
P=1-\exp \left(-\frac{7}{9} X_{0}\right)
$$

where $X_{0}$ are radiation lengths. If we were $100 \%$ efficient, the efficiency of identifying photons after conversion would be 1 minus the conversion probability. Since the amount of material is about $20 \%$ of a radiation length in the tracking chamber, a $1 \%$ uncertainty on the 
amount of material corresponds to a $\approx 0.2 \%$ uncertainty on the reconstruction efficiency $\left(\Delta_{e f f}\right) . \Delta_{e f f}$ is very small compared to the other uncertainties in this measurement and we choose to ignore it.

\section{- Systematics due to the Trigger Efficiency}

The determination of the trigger efficiency could be affected by residual background in our sample [52]. We have studied the effect of any possible contamination from fake electrons by using the events in the sideband region of the $Z$ mass window: $61<M_{Z}<71 \mathrm{GeV} / \mathrm{c}^{2}$ and $111<M_{Z}<121 \mathrm{GeV} / \mathrm{c}^{2}$. The trigger efficiency in the sideband was consistent with that in the signal region for $p_{T}>30 \mathrm{GeV} / \mathrm{c}$. We also measured the PHOTON_25_ISO trigger efficiency using electrons from $W$ events. In this case the electrons are required to be tight, the $W$ mass must be within 50 and $100 \mathrm{GeV} / \mathrm{c}^{2}$, and the QCD background is rejected by applying $M E T<25 \mathrm{GeV}$. This sample is likely to contain more residual background than the $Z$ sample, but still gives $\sim 100 \%$ efficiency (Fig. 5.6). The SUPER_PHOTON70 efficiency is always $100 \%$. We checked that we obtain the same result measuring it as relative to the PHOTON_25_ISO trigger sample. We conclude there are no variations in the triggers efficiencies and therefore any systematic uncertainty caused by the triggers efficiencies is negligible.

\subsection{Total Systematic Uncertainty}

The total systematic uncertainty and its different contributions are displayed in Fig. 5.7 and summarized in Tables 5.2 and 5.3 for every $p_{T}$ bin. The systematic uncertainty in the measurement is of $10 \%$ at low $p_{T}$ and increases to $15 \%$ at high $p_{T}$. The largest sources of uncertainty in the measurement come from the signal fraction in the first $p_{T}$ bins and the photon energy scale at high $p_{T}$. The uncertainty in the luminosity measurement is of $6 \%$ and is kept separate. 


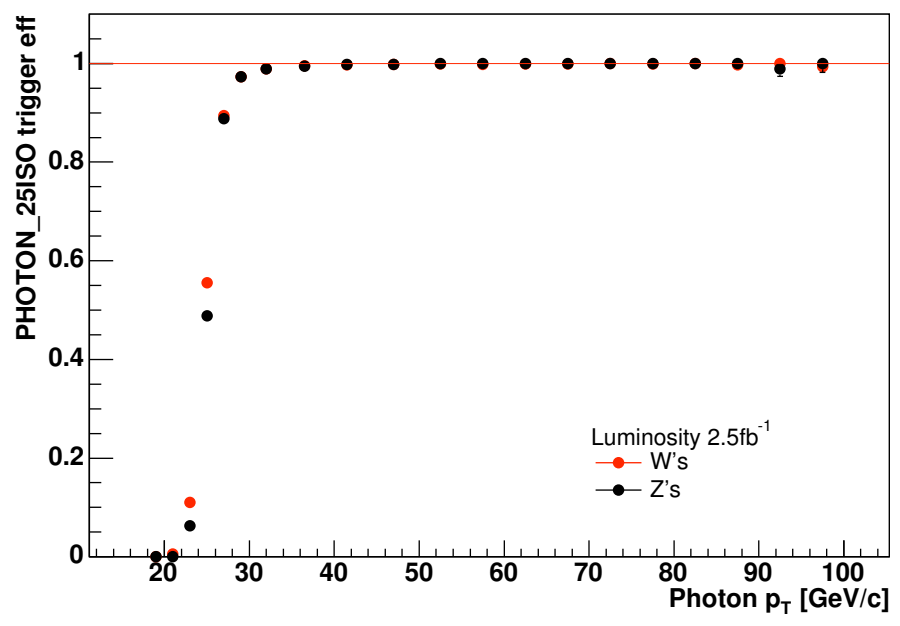

Figure 5.6: Comparison of the PHOTON_25_ISO trigger efficiencies obtained using electrons from Z's and electrons from W's as a function of the photon $p_{T}$.

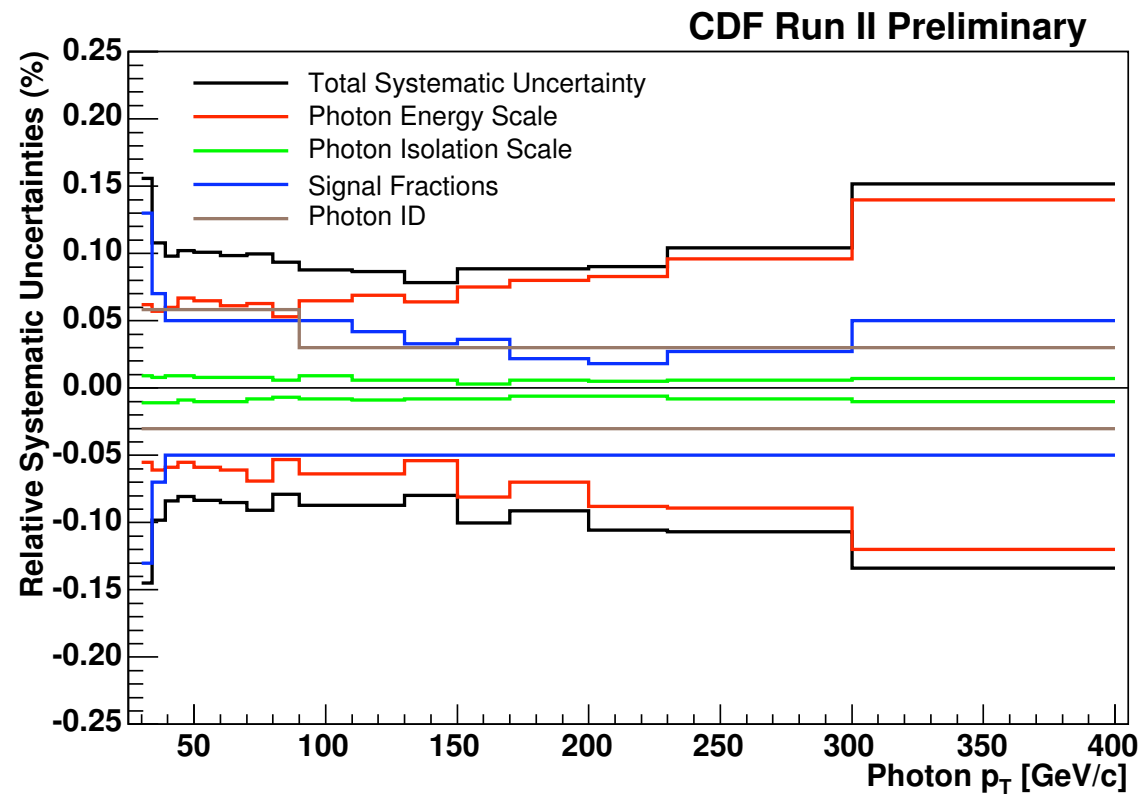

Figure 5.7: Total systematic uncertainty and its relative contributions as a function of the $p_{T}$ of the photon. The systematic uncertainties are asymmetric, and its different contributions are detailed in Tables 5.2 and 5.3. 


\begin{tabular}{c|rcccc}
\hline \hline$p_{T}^{\gamma}[\mathrm{GeV} / \mathrm{c}]$ & Total Positive & $\mathcal{F}$ & photonID & $e-$ scale & iso-scale \\
\hline $30-34$ & 0.156 & 0.130 & 0.058 & 0.062 & 0.009 \\
$34-39$ & 0.108 & 0.070 & 0.058 & 0.057 & 0.008 \\
$39-44$ & 0.098 & 0.050 & 0.058 & 0.060 & 0.009 \\
$44-50$ & 0.102 & 0.050 & 0.058 & 0.067 & 0.009 \\
$50-60$ & 0.101 & 0.050 & 0.058 & 0.065 & 0.008 \\
$60-70$ & 0.098 & 0.050 & 0.058 & 0.061 & 0.008 \\
$70-80$ & 0.100 & 0.050 & 0.058 & 0.063 & 0.008 \\
$80-90$ & 0.094 & 0.050 & 0.058 & 0.053 & 0.006 \\
$90-110$ & 0.088 & 0.050 & 0.030 & 0.065 & 0.009 \\
$110-130$ & 0.086 & 0.042 & 0.030 & 0.069 & 0.006 \\
$130-150$ & 0.078 & 0.033 & 0.030 & 0.064 & 0.006 \\
$150-170$ & 0.089 & 0.036 & 0.030 & 0.075 & 0.003 \\
$170-200$ & 0.088 & 0.022 & 0.030 & 0.080 & 0.006 \\
$200-230$ & 0.090 & 0.018 & 0.030 & 0.083 & 0.005 \\
$230-300$ & 0.104 & 0.027 & 0.030 & 0.096 & 0.006 \\
$300-400$ & 0.152 & 0.050 & 0.030 & 0.140 & 0.007 \\
\hline \hline
\end{tabular}

Table 5.2: Positive systematic uncertainties (in $\%$ ) for every $p_{T}$ bin. $\mathcal{F}$ is for signal fraction (blue line in Fig. 5.7), photon ID includes both the uncertainty due to the acceptance and the CES $\chi^{2}$ uncertainty (brown line in Fig. 5.7), and $e-$ scale and iso-scale are the energy and the isolation scales uncertainties (red and green lines respectively in Fig. 5.7). 


\begin{tabular}{c|rcccc}
\hline \hline$p_{T}^{\gamma}[\mathrm{GeV} / \mathrm{c}]$ & Total Negative & $\mathcal{F}$ & photonID & $e-$ scale & iso-scale \\
\hline $30-34$ & 0.145 & 0.13 & 0.03 & 0.055 & 0.011 \\
$34-39$ & 0.098 & 0.07 & 0.03 & 0.061 & 0.011 \\
$39-44$ & 0.084 & 0.05 & 0.03 & 0.059 & 0.011 \\
$44-50$ & 0.081 & 0.05 & 0.03 & 0.055 & 0.009 \\
$50-60$ & 0.084 & 0.05 & 0.03 & 0.059 & 0.010 \\
$60-70$ & 0.085 & 0.05 & 0.03 & 0.061 & 0.010 \\
$70-80$ & 0.091 & 0.05 & 0.03 & 0.069 & 0.008 \\
$80-90$ & 0.079 & 0.05 & 0.03 & 0.053 & 0.007 \\
$90-110$ & 0.087 & 0.05 & 0.03 & 0.064 & 0.008 \\
$110-130$ & 0.087 & 0.05 & 0.03 & 0.064 & 0.009 \\
$130-150$ & 0.080 & 0.05 & 0.03 & 0.054 & 0.008 \\
$150-170$ & 0.100 & 0.05 & 0.03 & 0.081 & 0.008 \\
$170-200$ & 0.091 & 0.05 & 0.03 & 0.070 & 0.006 \\
$200-230$ & 0.106 & 0.05 & 0.03 & 0.088 & 0.006 \\
$230-300$ & 0.107 & 0.05 & 0.03 & 0.089 & 0.008 \\
$300-400$ & 0.134 & 0.05 & 0.03 & 0.120 & 0.010 \\
\hline \hline
\end{tabular}

Table 5.3: Negative systematic uncertainties (in $\%$ ) for every $p_{T}$ bin. $\mathcal{F}$ is for signal fraction (blue line in Fig. 5.7), photon ID includes both the uncertainty due to the acceptance and the CES $\chi^{2}$ uncertainty (brown line in Fig. 5.7), and $e-$ scale and iso-scale are the energy and the isolation scales uncertainties (red and green lines respectively in Fig. 5.7). 


\section{Chapter 6}

\section{Results}

In this chapter we compare the measured inclusive and isolated prompt photon cross section to NLO perturbative QCD predictions. The theoretical predictions are corrected for non-perturbative contributions estimated from PYTHIA generated samples with different sets of parameters to control the underlying event activity.

\subsection{JETPHOX}

The results are compared to NLO pQCD predictions as determined using the JETPHOX program [57]. The calculation includes both direct processes, where the photon is produced through $q \bar{q}$ annihilation and QCD Compton scattering, and fragmentation processes, where the photon results from collinear fragmentation of partons with large transverse momentum.

The calculation is done in two parts. The first is the full NLO $\gamma j, \gamma j j$ matrix element. The second part is a NLO matrix element for $j j$ and $j j j$ final states, followed by a process of jets fragmenting to photons. This last step is performed by a Monte Carlo process. It must be noted that the separation between direct and fragmentation processes has no physical meaning beyond LO; at NLO accuracy, diagrams for the direct process contribute to the fragmentation process (see Section 2.2.1 for more details on this). The predictions are computed using $\mu_{\mathrm{R}}=\mu_{\mathrm{F}}=\mu_{\mathrm{f}}=p_{T}^{\gamma}$, CTEQ6.1M PDF's [58] and NLO fragmentation functions by Bouhris et al. [59].

JETPHOX allows arbitrary cuts on isolation energy around the photon. The nominal theoretical predictions are computed requiring a maximum of $2 \mathrm{GeV}$ in a cone of radius 0.4 around the 
photon. We have studied the effect of the isolation cut in the predicted cross section by changing the isolation cut from $0.25 \mathrm{GeV}$ to $4 \mathrm{GeV}$, which changes the theoretical predictions by less than 2\% (see Fig. 6.1). The theoretical predictions do not include the non perturbative effects of the underlying event and of the fragmentation of a parton into hadrons. This is addressed in Section 6.1.3.

\subsubsection{PDF Uncertainty}

The predicted cross section depends on the particular PDFs employed in the calculation. The uncertainty on the PDF is calculated with the Hessian method [12], where both the positive and negative deviations of the CTEQ6.1M PDF fit parameters (20 eigenvectors) are considered. Asymmetric uncertainties are obtained by summing in quadrature the maximal deviation in each direction associated to each of the 20 eigenvectors. If both deviations of a given eigenvector are in the same direction, only the largest deviation from the nominal value is considered. The PDF uncertainties vary between $5 \%$ and $15 \%$ as the photon transverse momentum increases.

\subsubsection{Dependence on the renormalization, factorization and fragmentation scales}

The changes in the theoretical cross section due to the variation of the scale provide an estimation of the size of the higher order terms that have not been considered in the fixed-order calculation. To measure the dependence of the prediction on the renormalization, factorization and fragmentation scales, they have been varied from half the value of the nominal scale $p_{T}^{\gamma} / 2$ to twice its value $2 p_{T}^{\gamma}$. This variation changes the theoretical prediction by around $15 \%$ at low $p_{T}$, decreasing to $8 \%$ at high $p_{T}$.

\subsubsection{Non-pQCD contributions}

The theoretical prediction does not account for non-perturbative effects due to the presence of the underlying event and of the fragmentation of the parton into hadrons after the parton shower. Therefore, in order to provide a fair comparison between data and theory, the latter needs to be corrected to include these effects. The underlying event activity is due to soft interactions between beam remnants, which add extra energy inside the isolation cone. The hadronization of the partons introduces some transverse momentum to the hadrons with respect to the original parton direction, which could also add some energy into the isolation cone. To estimate the effect 

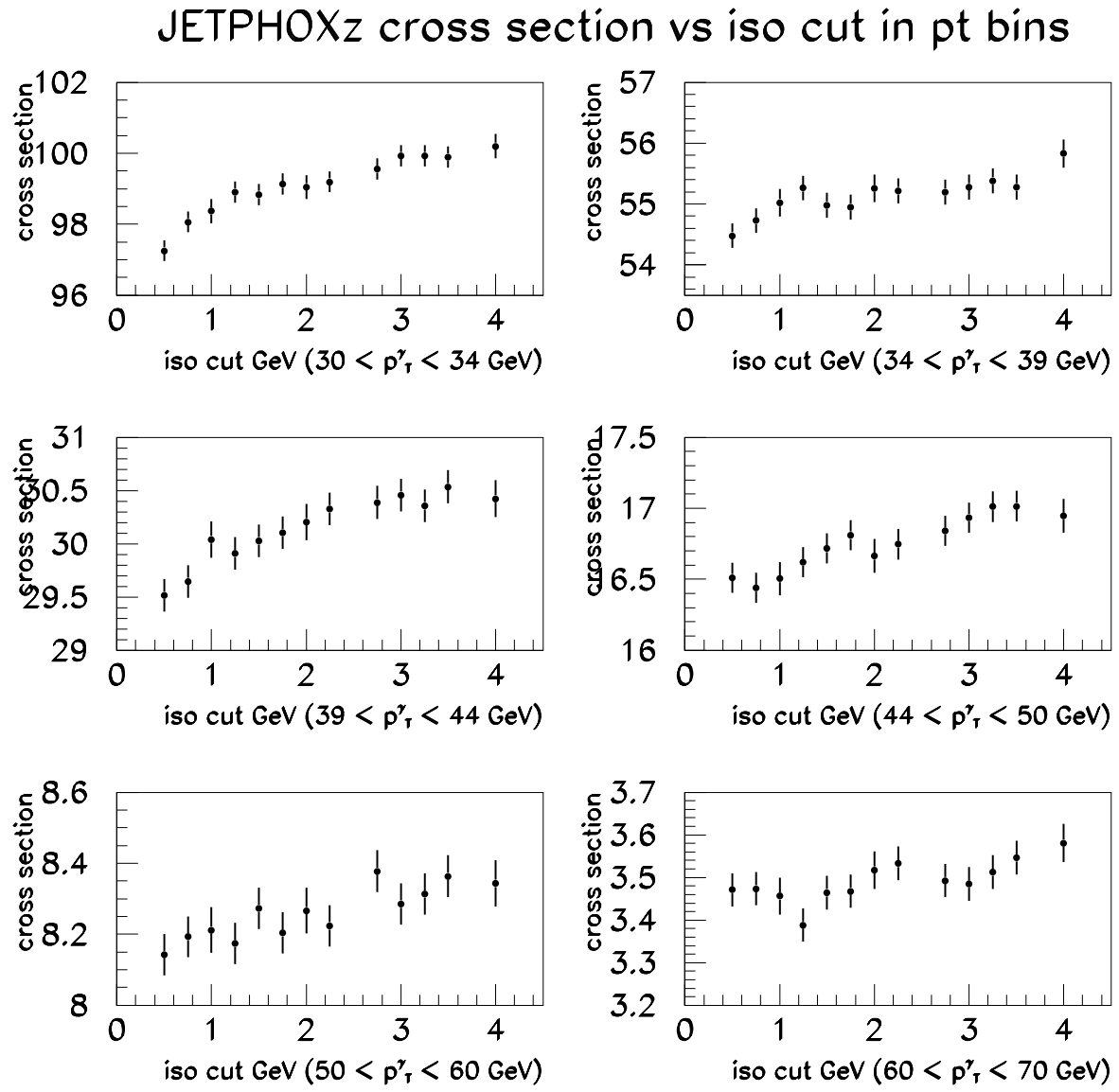

Figure 6.1: The cross section as predicted by JETPHOX as a function of the isolation transverse energy in a cone $R=0.4$ around the photon for different bins of photon transverse momentum.

of these contributions, we generate two different PYTHIA samples using two different sets of parameters for the underlying event, Tune A [29] and Tune DW [30], and compare the parton level cross sections with and without the non-pQCD contributions for each set. The correction is the averaged mean of the effect given by the two different sets of tunes, with a systematic 
uncertainty equal to the difference between the mean and the two extremes.

$$
C_{U E}=0.913 \pm 0.004(\text { stat }) \pm 0.03(\text { sys })
$$

The ratios of the cross sections with and without underlying event are shown in Fig. 6.2, for Tune A, and in Fig. 6.3, for Tune DW. As expected, when the non perturbative contributions are taken into account, the cross section decreases, as the $E_{T}^{i s o}<2 \mathrm{GeV}$ cut removes more events.

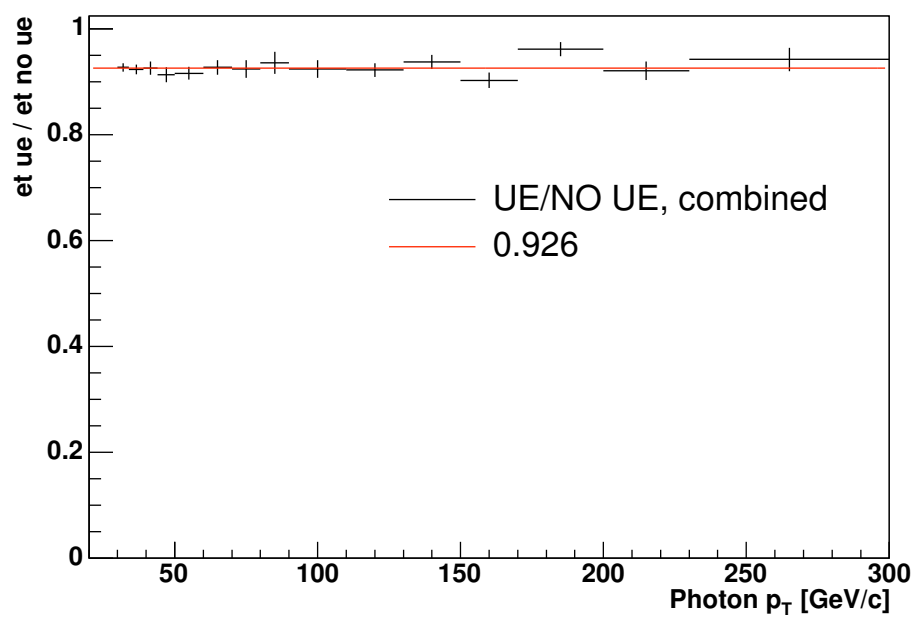

Figure 6.2: Ratio between the parton level cross section including the non-pQCD contributions and without including these contributions as predicted by PYTHIA Tune A samples. The correction to the JETPHOX theoretical predictions is given by the mean of the effects estimated with the Tune A set and with the Tune DW set.

\subsection{The Cross Section Result}

The inclusive photon cross section is defined as a function of to the $p_{T}$ of the photon according to the following equation:

$$
\frac{d \sigma}{d p_{T} d \eta}=\frac{N_{\text {data }} \mathcal{F}}{\Delta p_{T} \Delta \eta \mathcal{L} \varepsilon_{T} U}
$$

where $N_{\text {data }}$ is the number of photon candidates in a given $p_{T}$ bin and $\mathcal{F}$ is the corresponding fraction of prompt photons. $\Delta p_{T}(\Delta \eta)$ is the size of the $p_{T}(\eta)$ bin, and $\varepsilon_{T}$ is the trigger efficiency. $U$ are the unfolding factors, which correct the measured cross section for acceptance, efficiency 


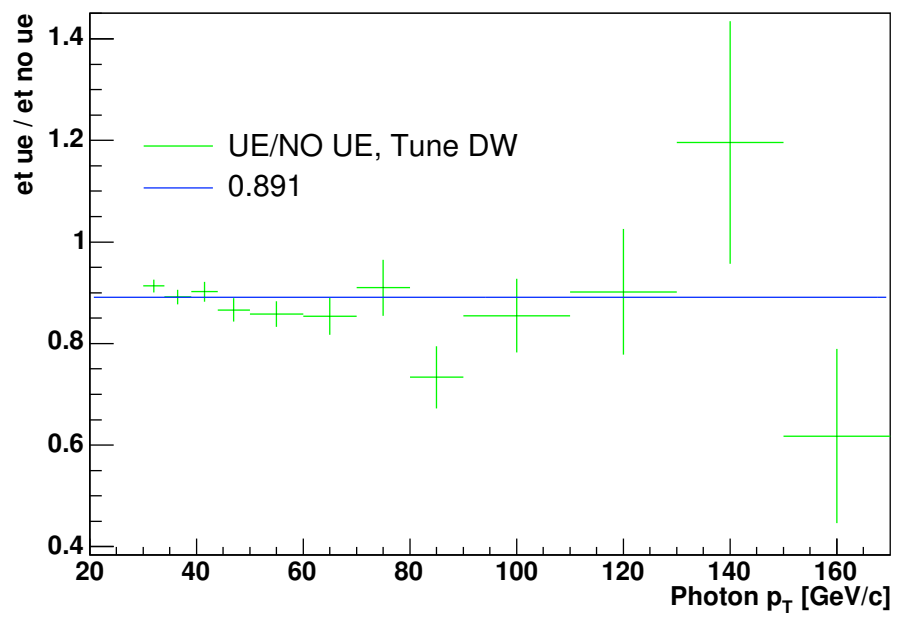

Figure 6.3: Ratio between the parton level cross section including the non-pQCD contributions and without including these contributions as predicted by PYTHIA Tune DW samples. The correction to the JETPHOX theoretical predictions is given by the mean of the effects estimated with the Tune A set and with the Tune DW set.

of the photon selection, and resolution effects back to the hadron level (see Section 4.7). $\mathcal{L}$ is the total integrated luminosity, $\mathcal{L}=2.5 \pm 0.1 \mathrm{fb}^{-1}$.

Fig. 6.4 shows the differential cross section for central $(|\eta|<1.0)$ and isolated $\left(E_{T}^{i s o}<2.0 \mathrm{GeV}\right)$ photons as a function of the $p_{T}$ of the photon. The measurement tests the $\mathrm{pQCD}$ predictions over 6 orders of magnitude and up to transverse momentum of the photon of about $400 \mathrm{GeV} / \mathrm{c}$. The statistical uncertainties in the measurement are represented by the error bars associated to the data points, and also take into account the small effect of the limited statistics in the Monte Carlo samples used in the analysis. The total systematic uncertainty in the measurement, as discussed in Chapter 5, is represented by the yellow band and varies between 10 and $15 \%$ in the whole measured range. An additional $6 \%$ uncertainty due to the luminosity is not included in the Figures.

The ratio of the measurement to the theoretical predictions is presented in Fig. 6.5. The theory predictions have been corrected for the non-pQCD effects, as shown in the bottom part of Fig. 6.6. The uncertainty in the theory due to the PDF is represented by the blue dotted-dashed line, while the effect of the scale variation is given by the red dashed line in the Figures. The theoretical predictions describe well the data except for $p_{T}<40 \mathrm{GeV} / \mathrm{c}$, where the measured cross section has 
different shape and normalization to that of the theory. These differences are not covered by the uncertainties in the theory or in the measurement. In Fig. 6.7 the measured cross section is compared to the predictions obtained using MRST04 [11] parametrization of the PDF, where the ratio of the predictions with CTEQ6.1M to those from MRST04 is also shown. This ratio is well within the experimental and the theoretical uncertainties, though it presents a slightly different slope with the photon $p_{T}$. The differences at low $p_{T}$ have been observed in previous measurements of the photon cross section [60], both for collider and fixed-target experiments. These observations motivated the introduction of soft resummation effects in the theoretical predictions [19], but it is not likely it would affect $p_{T}>30 \mathrm{GeV} / \mathrm{c}$ [20]. For $p_{T}>40 \mathrm{GeV} / \mathrm{c}$ we observe good agreement between the data and the theoretical predictions from JETPHOX, and therefore our data might be useful in this range to constrain the gluon PDF.

The measured cross section with the statistical and systematic uncertainties, together with the number of photon candidates, signal fraction, trigger efficiencies and unfolding factors, is detailed for every $p_{T}$ bin in Table 6.1. The last column of Table 6.1 shows the quoted theoretical prediction. 


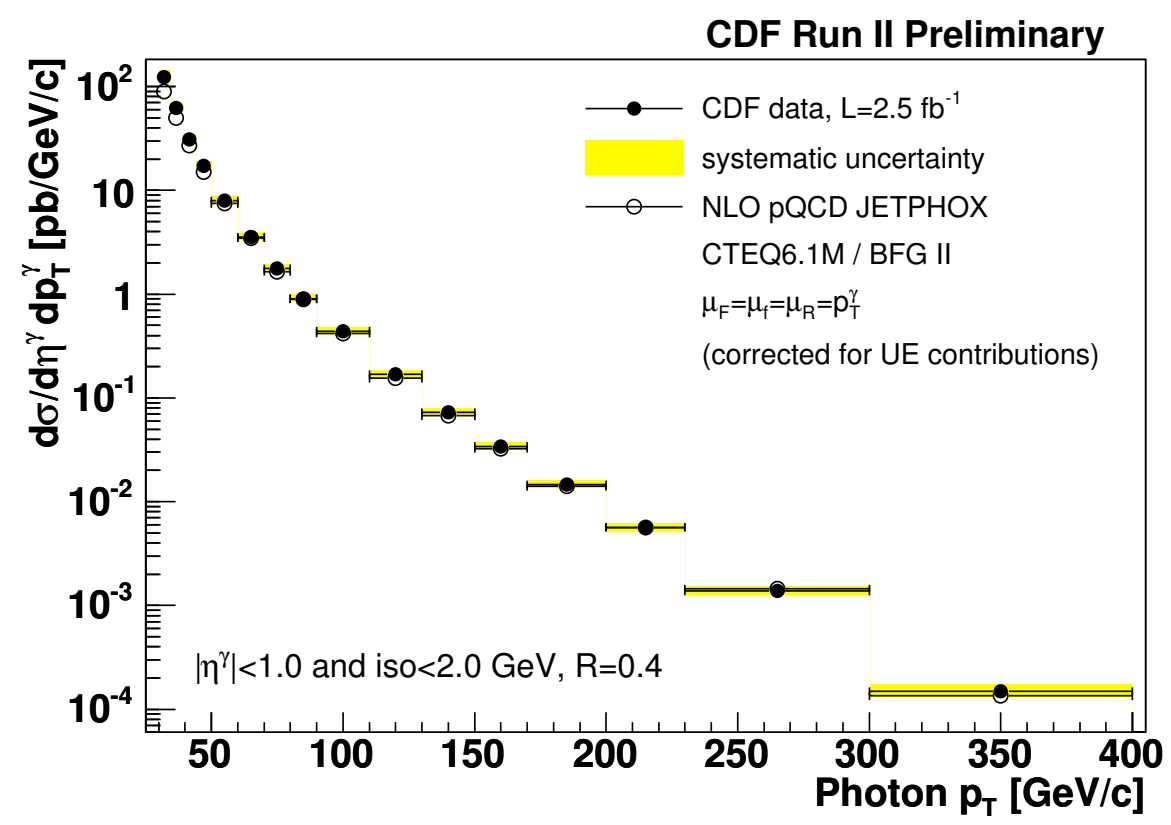

Figure 6.4: Measured inclusive isolated photon cross section as a function of the photon $p_{T}$ compared to NLO pQCD predictions corrected for non-pQCD contributions. The yellow band includes the total systematic uncertainty on the measurement, except for the $6 \%$ luminosity uncertainty. 


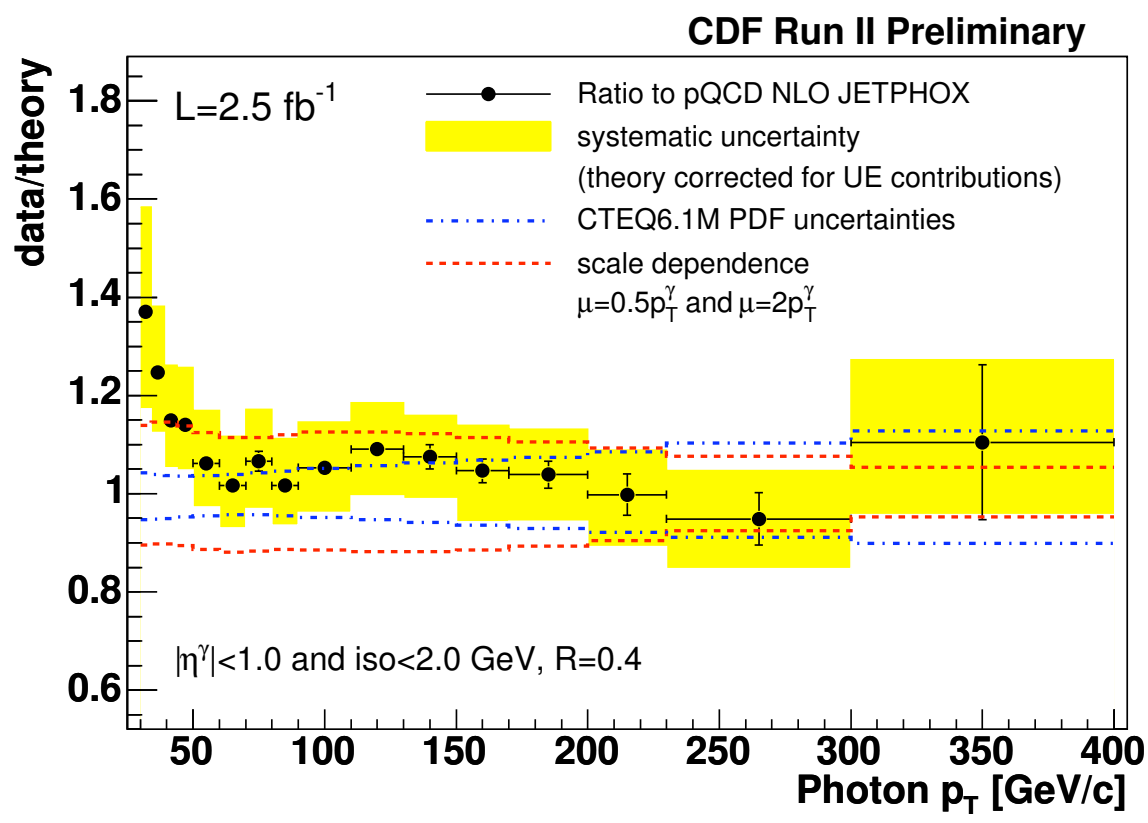

Figure 6.5: Ratio DATA/THEORY as a function of the $p_{T}$ of the photon. The dot-dashed blue lines indicate the PDF uncertainty, and the dashed red line indicates the variation of the cross section when the renormalization, factorization and fragmentation scales are varied from $\mu=p_{T}$ to $\mu=p_{T} / 2$ and $\mu=2 P_{T}$. The theoretical prediction is corrected for the non-pQCD contributions. 


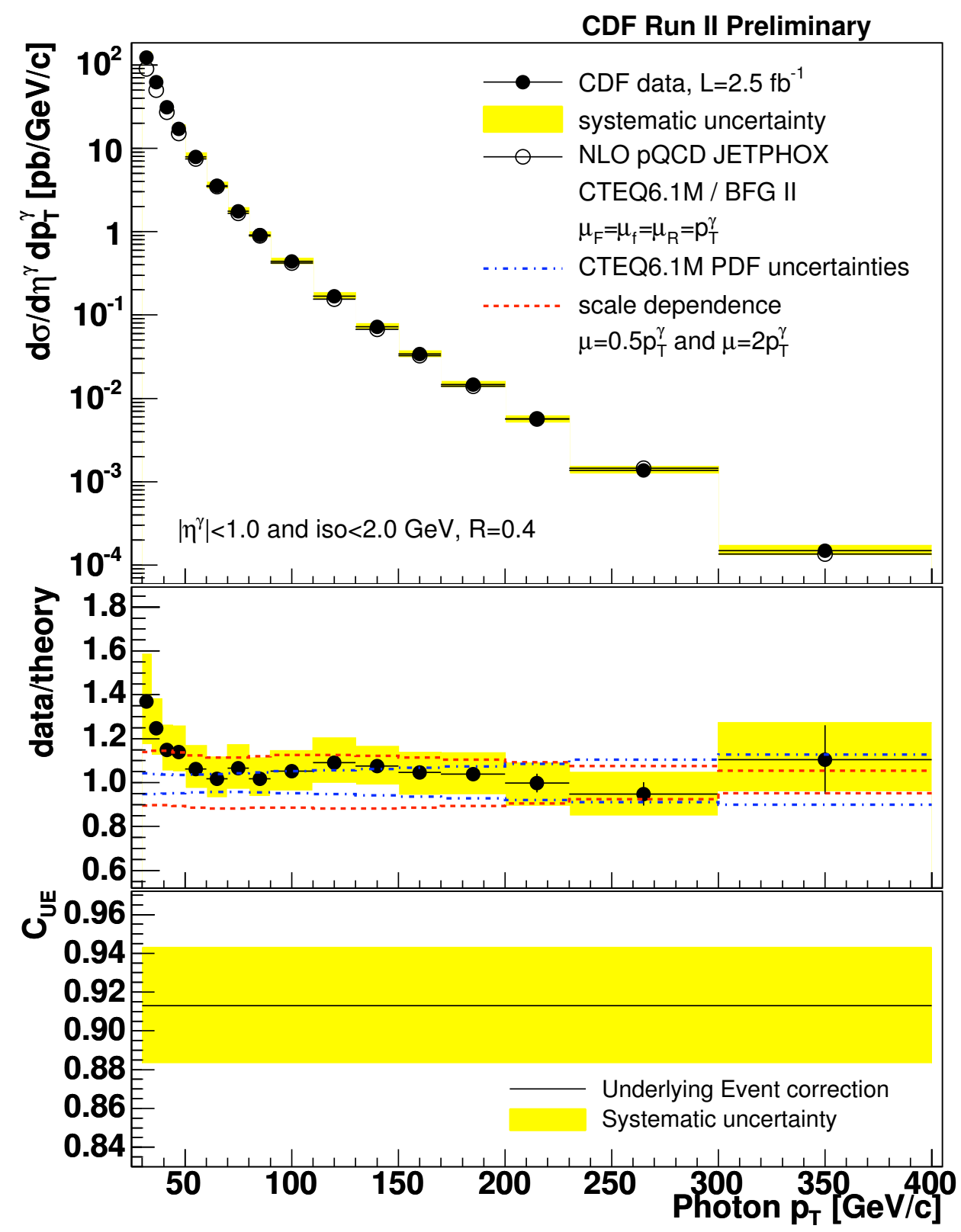

Figure 6.6: Measured inclusive isolated photon cross section as a function of the photon $p_{T}$ compared to NLO pQCD predictions (top). The yellow band includes the total systematic uncertainty on the measurement except for the $6 \%$ luminosity uncertainty. The ratio DATA/THEORY as a function of the $p_{T}$ of the photon is presented below. The dot-dashed blue lines indicate the PDF uncertainty, and the dashed red lines indicate the variation of the cross section when the renormalization, factorization and fragmentation scales are varied from $\mu=p_{T}$ to $\mu=p_{T} / 2$ and $\mu=2 P_{T}$. The theoretical prediction is corrected for the non-pQCD contributions by $C_{U E}=0.913 \pm 0.004$ (stat) \pm 0.03 (sys), as shown in the bottom plot. 


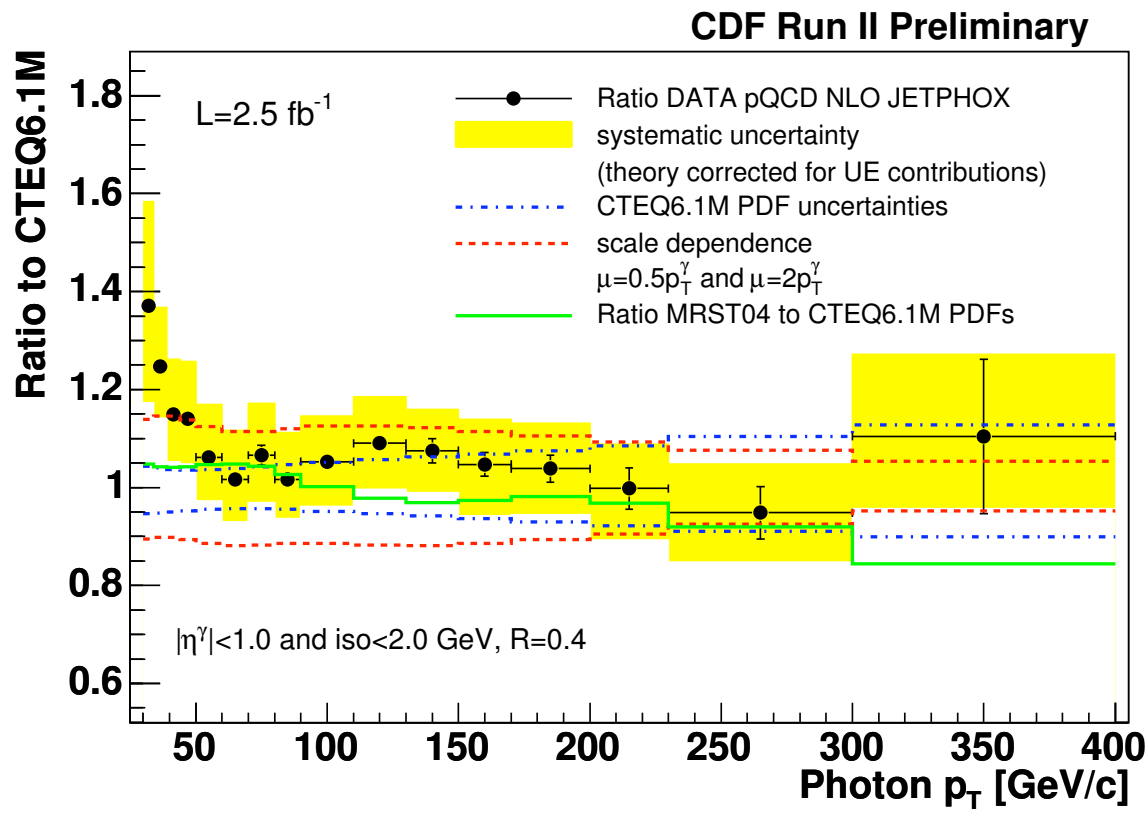

Figure 6.7: Ratio of data to theory predictions with CTEQ6.1M PDFs as a function of the $p_{T}$ of the photon (black dots) compared to the ratio of the predictions with CTEQ6.1M PDF to the predictions obtained with MRST04 PDF parametrization, indicated by the green line. The uncertainties due to the CTEQ6.1M PDF are indicated by the dot-dashed line, while the scale dependence is given by the dashed red line. The systematic uncertainties in the measurement are indicated by the yellow band. 


\begin{tabular}{c|rcc}
\hline \hline$p_{T}^{\gamma}[\mathrm{GeV} / \mathrm{c}]$ & $N_{\text {data }}$ & $d \sigma^{\gamma} / \mathrm{dp}_{T}^{\gamma} d \eta^{\gamma} \pm(\mathrm{stat}) \pm(\mathrm{sys})[\mathrm{pb} / \mathrm{GeV}]$ & JETPHOX $[\mathrm{pb} / \mathrm{GeV}]$ \\
\hline $30-34$ & 2117070 & $1.23 \times 10^{2} \pm 0.5_{-14.5}^{+15.6}$ & $8.98 \times 10^{1}$ \\
$34-39$ & 1313630 & $6.21 \times 10^{1} \pm 0.5_{-9.82}^{+10.8}$ & $4.98 \times 10^{1}$ \\
$39-44$ & 659088 & $3.10 \times 10^{1} \pm 0.8_{-8.37}^{+9.79}$ & $2.69 \times 10^{1}$ \\
$44-50$ & 412236 & $1.72 \times 10^{1} \pm 0.9_{-8.07}^{+10.2}$ & $1.51 \times 10^{1}$ \\
$50-60$ & 311244 & $7.93 \times 10^{0} \pm 1.1_{-8.36}^{+10.1}$ & $7.47 \times 10^{0}$ \\
$60-70$ & 129912 & $3.54 \times 10^{0} \pm 1.5_{-8.50}^{+9.84}$ & $3.49 \times 10^{0}$ \\
$70-80$ & 62017 & $1.76 \times 10^{0} \pm 1.9_{-9.07}^{+9.97}$ & $1.65 \times 10^{0}$ \\
$80-90$ & 32511 & $9.08 \times 10^{-1} \pm 1.6_{-7.91}^{+9.35}$ & $8.98 \times 10^{-1}$ \\
$90-110$ & 31284 & $4.41 \times 10^{-1} \pm 1.1_{-8.70}^{+8.78}$ & $4.19 \times 10^{-1}$ \\
$110-130$ & 11616 & $1.68 \times 10^{-1} \pm 1.5_{-8.71}^{+8.64}$ & $1.54 \times 10^{-1}$ \\
$130-150$ & 4918 & $7.25 \times 10^{-2} \pm 2.3_{-7.99}^{+7.82}$ & $6.74 \times 10^{-2}$ \\
$150-170$ & 2348 & $3.41 \times 10^{-2} \pm 2.3_{-10.0}^{+8.85}$ & $3.26 \times 10^{-2}$ \\
$170-200$ & 1497 & $1.46 \times 10^{-2} \pm 2.7_{-9.13}^{+8.84}$ & $1.41 \times 10^{-2}$ \\
$200-230$ & 587 & $5.66 \times 10^{-3} \pm 4.2_{-10.6}^{+9.02}$ & $5.67 \times 10^{-3}$ \\
$230-300$ & 331 & $1.38 \times 10^{-3} \pm 5.5_{-10.7}^{+10.4}$ & $1.45 \times 10^{-3}$ \\
$300-400$ & 51 & $1.49 \times 10^{-4} \pm 14.3_{-13.4}^{+15.2}$ & $1.35 \times 10^{-4}$ \\
\hline \hline
\end{tabular}

Table 6.1: The cross section result and the corresponding theoretical prediction. The latter does not include the correction due to the non pQCD effects, $C_{U E}=0.913 \pm 0.004$ (stat) \pm 0.03 (sys). The statistical and systematic uncertainties in the measurement are also presented, both in \%. The statistical errors come from the data, and also take into account the small effect of the limited statistics in the Monte Carlo samples employed for the unfolding factors (see Chapter 4), while the systematic uncertainties are asymmetric and are explained in detail in Chapter 5. The luminosity uncertainty, of 6\%, is not included in the Table. 



\section{Chapter 7}

\section{Conclusions}

This thesis presents the measurement of the inclusive photon cross section for photons with $|\eta|<1.0, p_{T}>30 \mathrm{GeV} / \mathrm{c}$ and isolation $<2 \mathrm{GeV}$ using $2.5 \mathrm{fb}^{-1}$ of data taken by the CDF detector between February 2002 and August 2007. This measurement includes 6 times more data than the last published result [3], extending its $p_{T}$ coverage by $100 \mathrm{GeV} / \mathrm{c}$. The cross section is measured up to $400 \mathrm{GeV} / \mathrm{c}$, and tests the theoretical predictions over 6 orders of magnitude, one order of magnitude more than in previous results.

The prompt photon cross section measurement offers a unique opportunity to test the photon tools over a large energy range. In this thesis we have presented a new technique to suppress the irreducible isolated photons from meson decays. The method consists of fitting the calorimeter isolation distribution in the data to pure signal and background templates for every bin in the photon $p_{T}$. The template method is simple and is based only on the calorimeter information. Moreover, it significantly reduces the systematic uncertainty associated to the photon purity. Previous Run I CDF measurements used techniques based on information collected by the CES and CPR detectors [5], which have considerable statistical dilution and systematics. With the method introduced in this thesis, the systematic uncertainty in the photon purity is reduced from $30 \%$ in the previous CDF Run I measurements to $5 \%$ at high $p_{T}$. With the improvement of the Monte Carlo simulations, the template method has the potential to become a powerful tool for future searches using photon signatures.

Data are unfolded back to hadron level to correct for efficiencies and detector acceptance and resolution effects using a bin-by-bin procedure implemented in a PYTHIA inclusive photon Monte Carlo sample. The unfolding factors do not present strong dependence on the photon transverse 
momentum, and vary between $64 \%$ and $69 \%$ in the $p_{T}$ considered.

We compare our results to the predictions given by JETPHOX using CTEQ6.1M PDF, BFGII fragmentation functions, and renormalization, fragmentation and factorization scales set equal to the transverse momentum of the photon. The theoretical predictions are corrected for non perturbative QCD effects. The systematic uncertainties in the data are around $13 \%$ at low $p_{T}$, dominated by the signal fractions, while at high $p_{T}$ they are about $15 \%$, mainly coming from the photon energy scale. The uncertainties in the theory are due to the PDF, of around $5 \%$ at low $p_{T}$ and increasing to about $15 \%$ at high $p_{T}$. The dependence left in the prediction due to the choice of the scales is of $15 \%$ at low $p_{T}$ and decreases to around $8 \%$ at high $p_{T}$.

We find agreement between data and theory above $40 \mathrm{GeV} / \mathrm{c}$. In the $p_{T}$ range until $150 \mathrm{GeV} / \mathrm{c}$, the prompt photon production is dominated by the QCD Compton diagram, while for higher $p_{T} \mathrm{~S}$ gluons substantially contribute to the prompt photon production though they are not the dominant contribution. Therefore, in the $p_{T}$ range above $40 \mathrm{GeV} / \mathrm{c}$, our measurement might be useful to constrain the gluon PDF. For $p_{T}$ until $40 \mathrm{GeV} / \mathrm{c}$ data shows an excess compared to the theory. This trend has been previously seen by many other experiments, both in colliders [2] and fixedtarget [4] data, and it is probably not due to systematic effects in the experimental method but more likely to other physics effects that are not accounted for in the theoretical calculations. There was an attempt to explain these effects, together with the deviations seen by some fixedtarget experiments, with the introduction of a new parameter in the calculations that accounted for soft gluon radiation effects in the initial state [17]. However, its effect is not believed to affect $p_{T}$ higher than $\sim 30 \mathrm{GeV} / \mathrm{c}$ [20]. The comparison to the predictions obtained using the MRST04 [11] parametrization for the PDF results is in agreement with CTEQ6.1M for the whole measured range, with similar shapes at low $p_{T}$, though in the whole $p_{T}$ range the ratio presents a slightly different slope that makes the cross section to decrease at high $p_{T}$.

Finally, the measurement has been approved by the CDF Collaboration and it is in process for a publication in Physics Review Letters. 


\section{Appendix A}

\section{Fit results using $\mathrm{Z}$ templates}

In this Appendix we present the fit results using as a signal templates the isolation of electrons in $Z \rightarrow e^{+} e^{-}$decays, which forms part of the various studies we performed to estimate the systematic uncertainty in the signal fractions. 

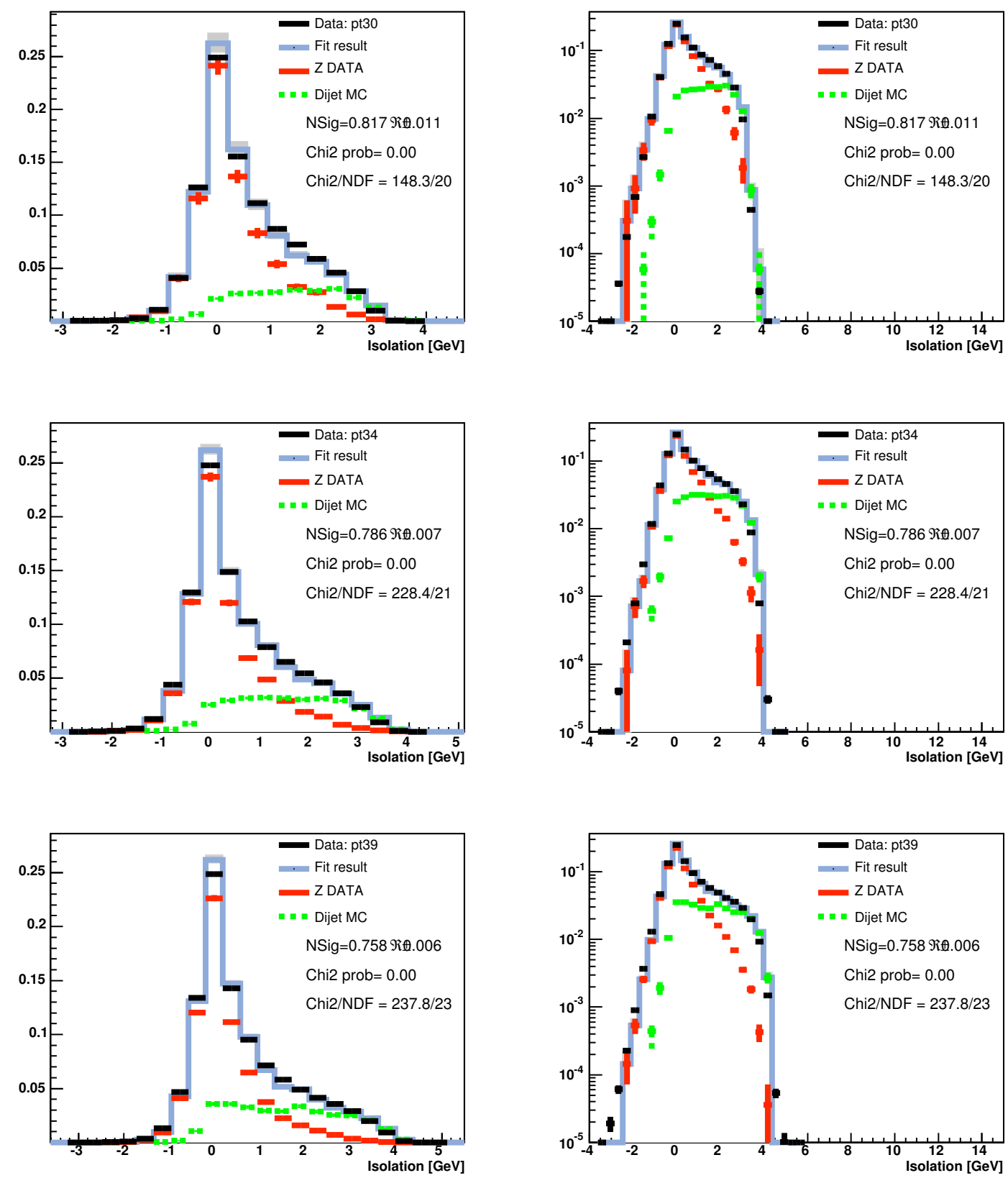

Figure A.1: Fits to the isolation distribution in bins of $p_{T}$ for $30<p_{T}<44 \mathrm{GeV}$. Left column shows the fit results in linear scale. Logarithmic scales are displayed in right column. The signal template is from $\mathrm{Z}$ data. The background template is from jet Monte Carlo with brem photons removed. 

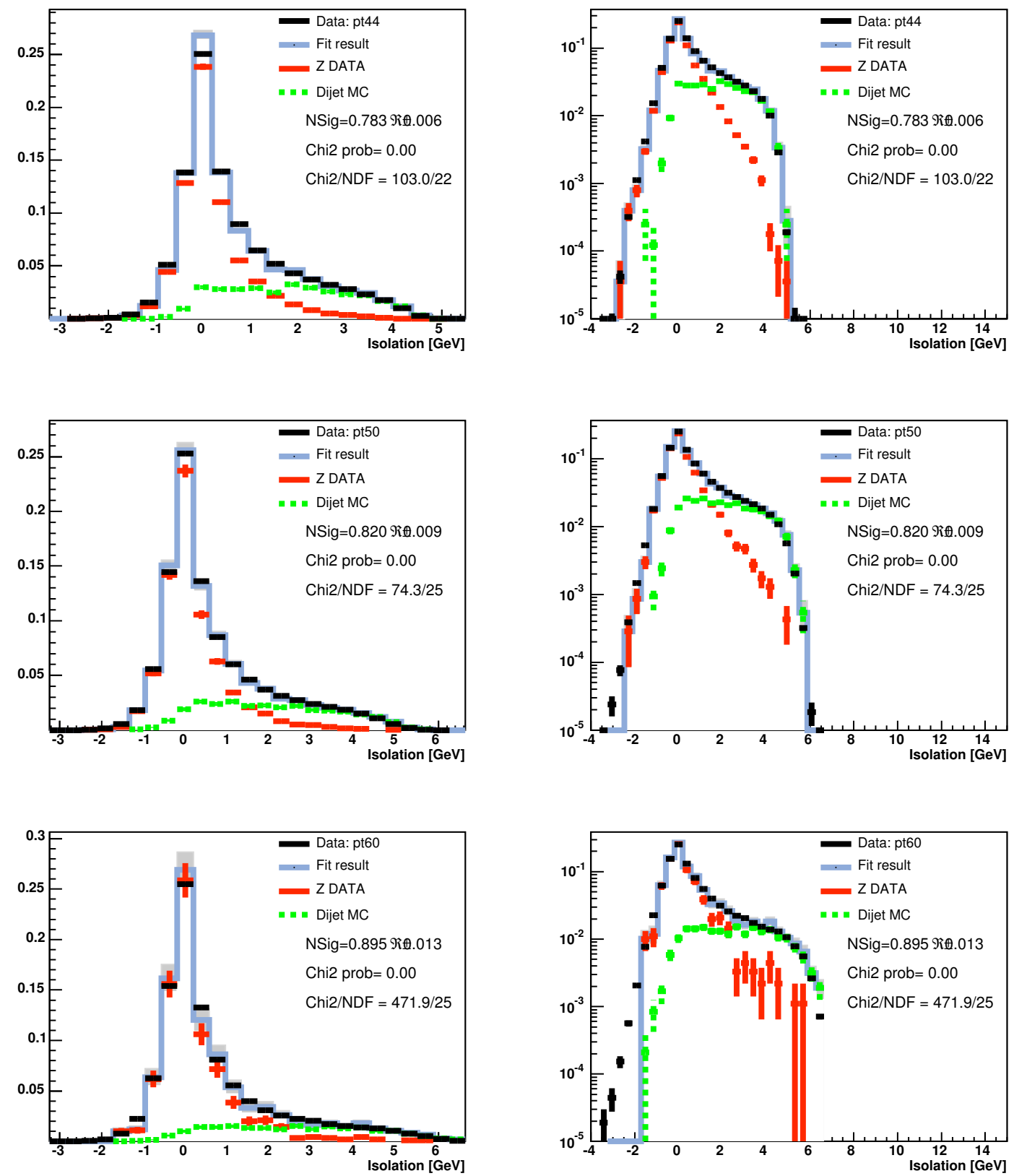

Figure A.2: Fits to the isolation distribution in bins of $p_{T}$ for $44<p_{T}<70 \mathrm{GeV}$. Left column shows the fit results in linear scale. Logarithmic scales are displayed in right column. The signal template is from $\mathrm{Z}$ data. The background template is from jet Monte Carlo with brem photons removed. 



\section{Appendix B}

\section{Fit results using corrected dijet MC templates}

In this Appendix we present the fit results using corrected dijet Monte Carlo templates for the background, which forms part of the various studies we performed to estimate the systematic uncertainty in the signal fractions. 

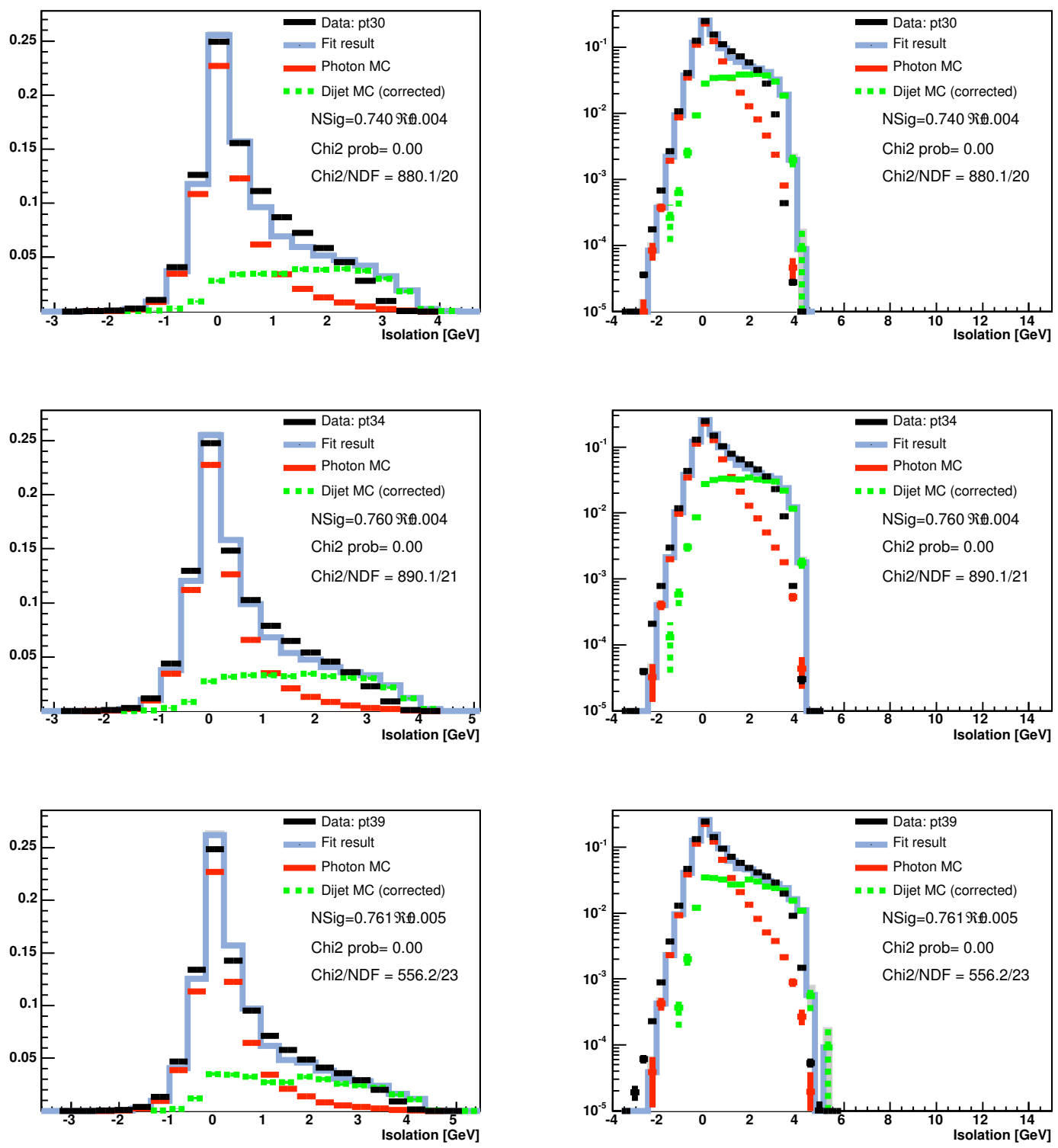

Figure B.1: Fits to the isolation distribution in bins of $p_{T}$ for $30<p_{T}<44 \mathrm{GeV}$. Left column shows the fit results in linear scale. Logarithmic scales are displayed in right column. The signal template is from photon Monte Carlo, the background template is from corrected jet Monte Carlo with brem photons removed. 

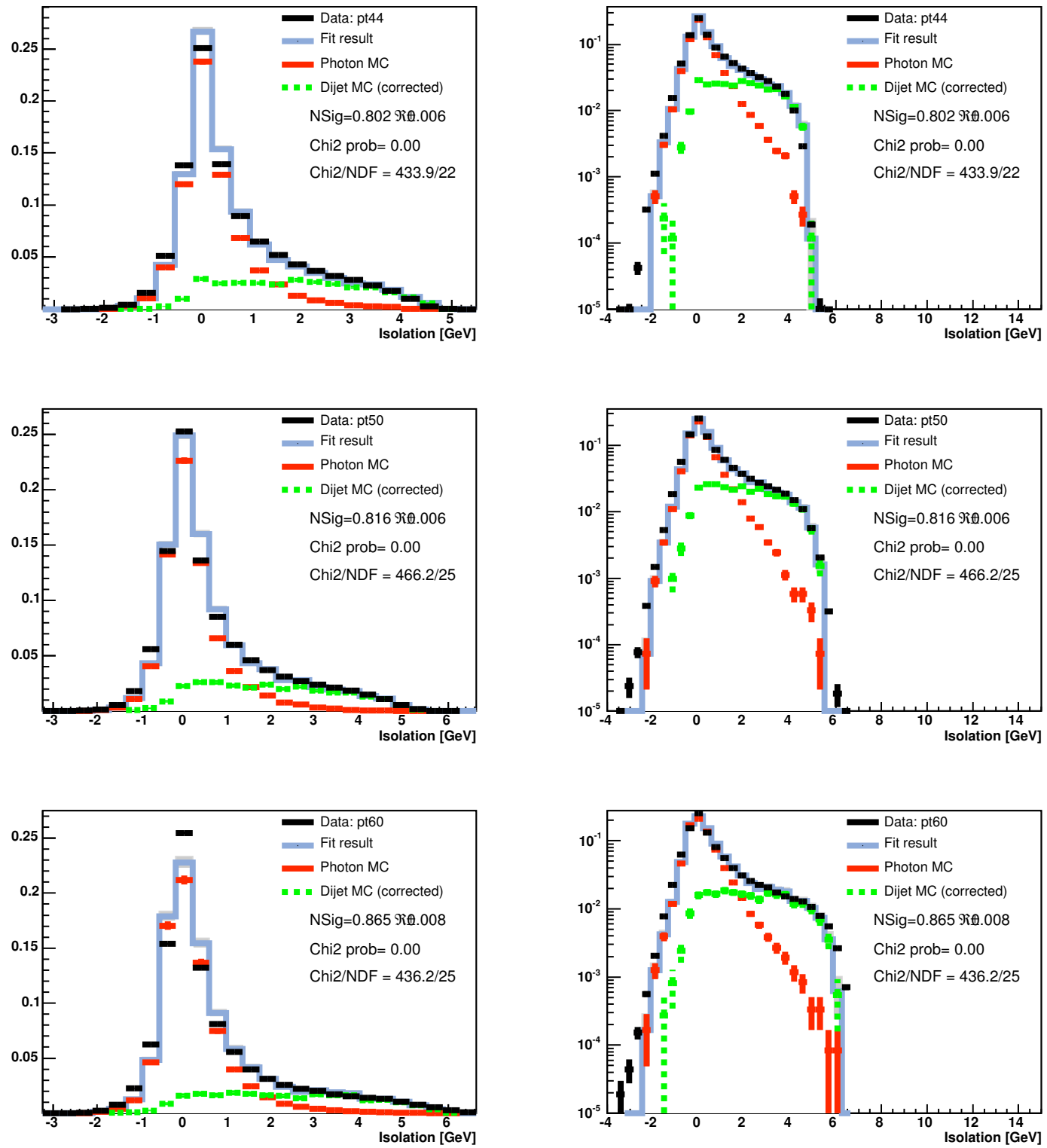

Figure B.2: Fits to the isolation distribution in bins of $p_{T}$ for $44<p_{T}<70 \mathrm{GeV}$. Left column shows the fit results in linear scale. Logarithmic scales are displayed in right column. The signal template is from photon Monte Carlo, the background template is from corrected jet Monte Carlo with brem photons removed. 

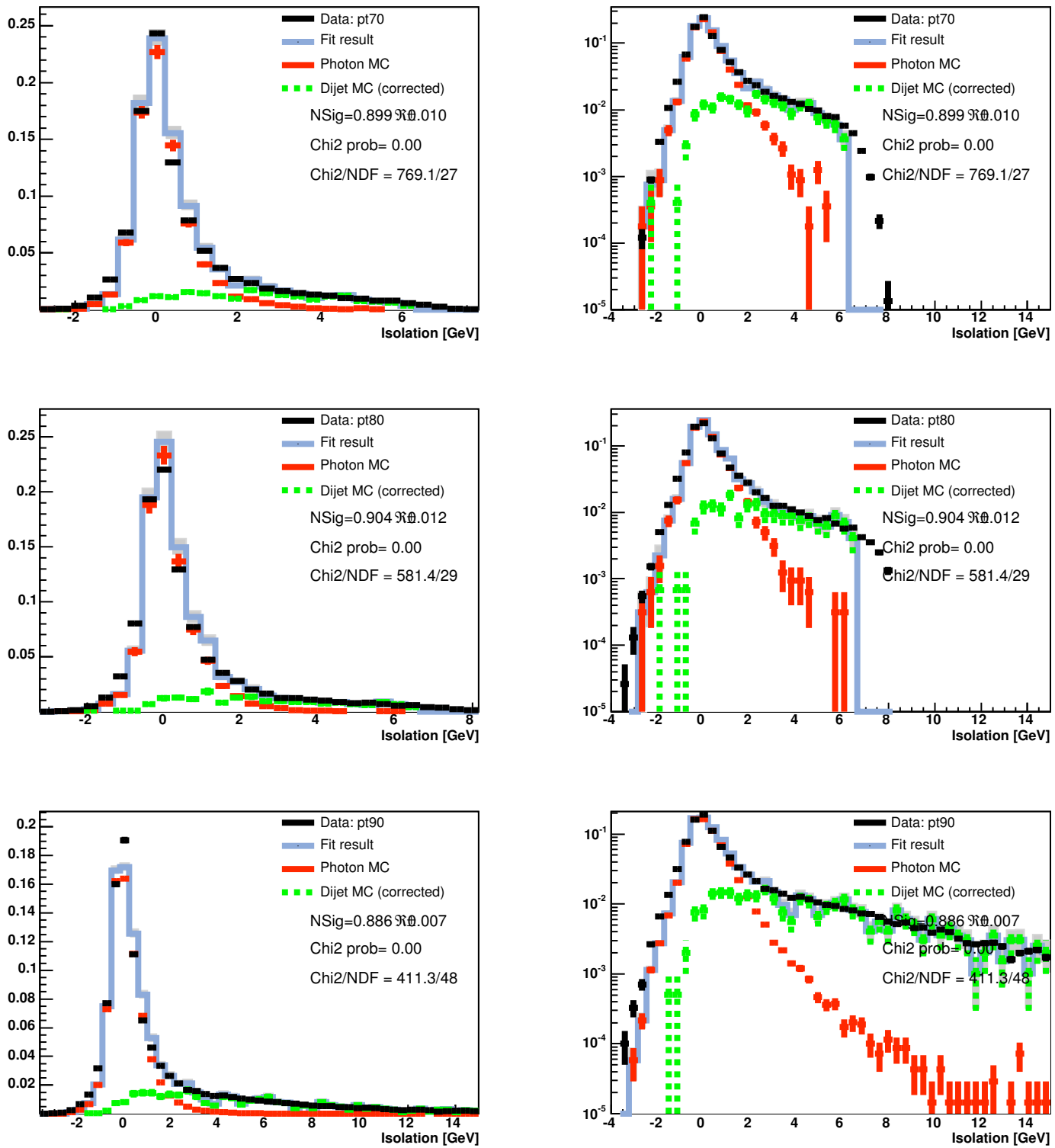

Figure B.3: Fits to the isolation distribution in bins of $p_{T}$ for $70<p_{T}<110 \mathrm{GeV}$. Left column shows the fit results in linear scale. Logarithmic scales are displayed in right column. The signal template is from photon Monte Carlo, the background template is from corrected jet Monte Carlo with brem photons removed. 

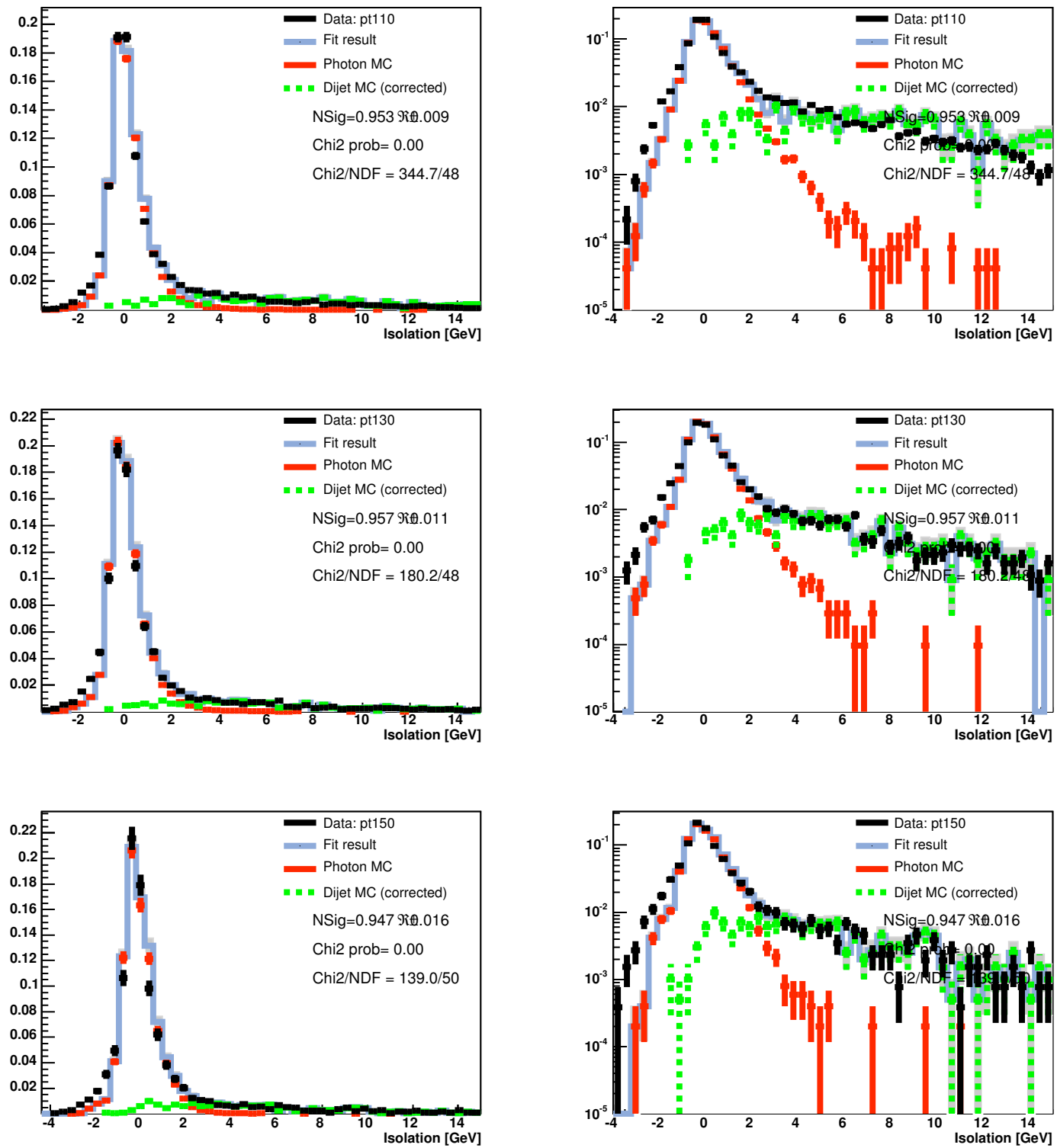

Figure B.4: Fits to the isolation distribution in bins of $p_{T}$ for $110<p_{T}<170 \mathrm{GeV}$. Left column shows the fit results in linear scale. Logarithmic scales are displayed in right column. The signal template is from photon Monte Carlo, the background template is from corrected jet Monte Carlo with brem photons removed. 

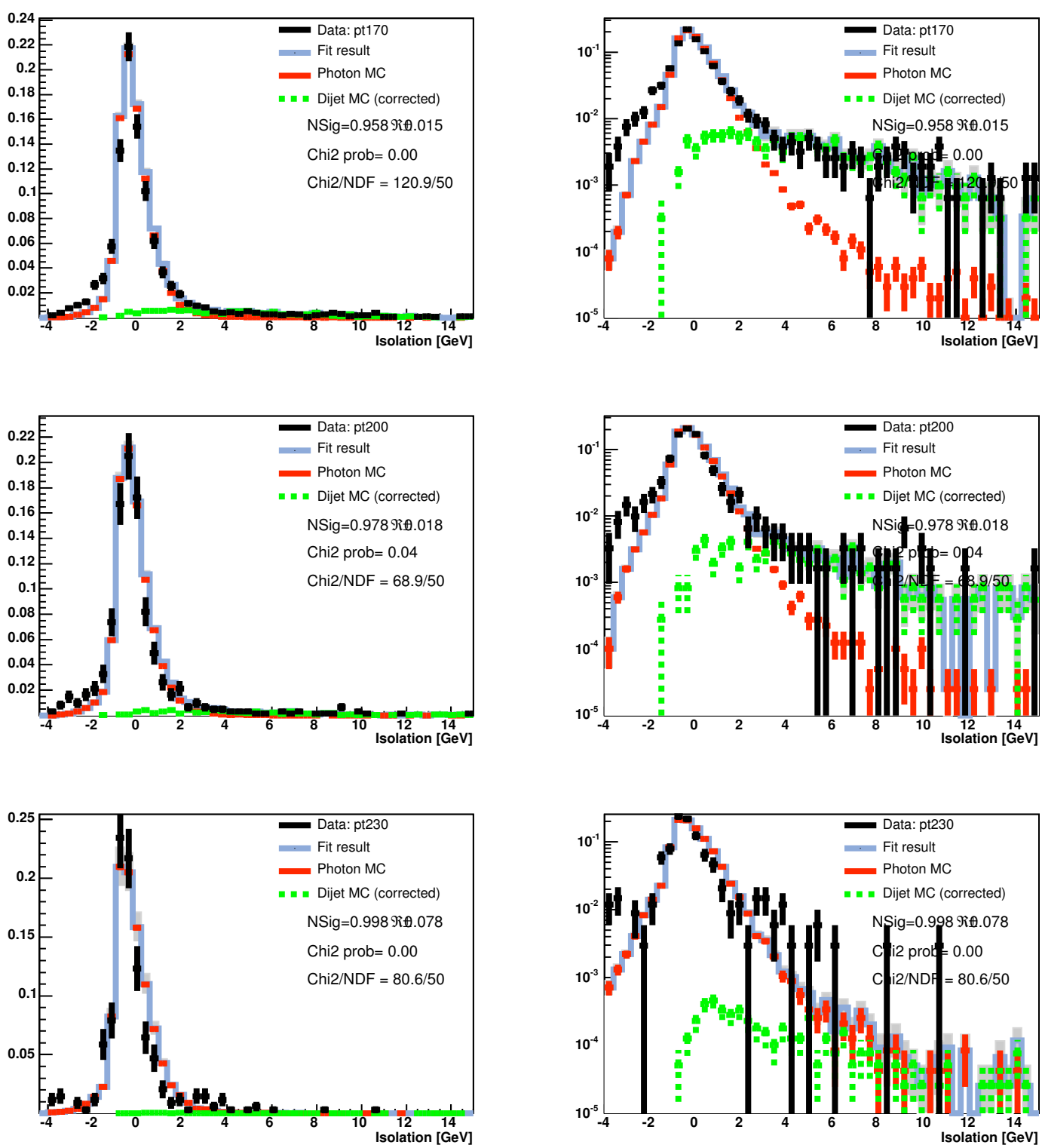

Figure B.5: Fits to the isolation distribution in bins of $p_{T}$ for $170<p_{T}<300 \mathrm{GeV}$. Left column shows the fit results in linear scale. Logarithmic scales are displayed in right column. The signal template is from photon Monte Carlo, the background template is from corrected jet Monte Carlo with brem photons removed. 

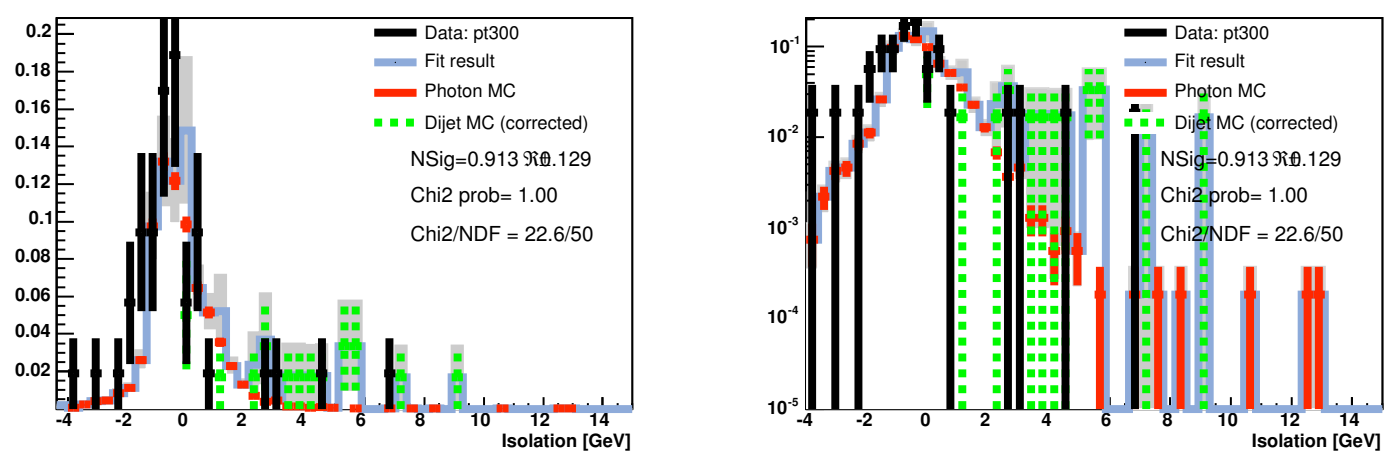

Figure B.6: Fits to the isolation distribution in bins of $p_{T}$ for $300<p_{T}<400 \mathrm{GeV}$. Left column shows the fit results in linear scale. Logarithmic scales are displayed in right column. The signal template is from photon Monte Carlo, the background template is from corrected jet Monte Carlo with brem photons removed. 



\section{Appendix C}

\section{Study of the Isolation in the Photon Monte Carlo}

For the fitting procedure in this analysis, we have to make an ad hoc correction to the isolation energy in the signal Monte Carlo template (Section 4.6). At high photon energies, the isolation in the photon Monte Carlo samples does not describe the trend observed in the data. In the Monte Carlo, the mean and the width of the isolation peak tend to increase with the photon energy, while in the data the peak is always well isolated. For energies below $70 \mathrm{GeV}$, the isolation distributions from data and Monte Carlo agree. Fig. C.1 shows the fit result using the original (before our correction) photon Monte Carlo template for photon $p_{T}$ between 110 and $130 \mathrm{GeV} / \mathrm{c}$.

The isolation energy comes from two components. The first one comes from the underlying event energy and the pile-up, whose contributions are constant with the photon $p_{T}$. The second component comes from leakage across the $\phi$ boundary between wedges. Since the EM clustering is done only in the $\eta$-direction, energy which leaks out of the shower laterally into the adjacent wedge can be included in the isolation cone but lost from the shower. This contribution depends on the energy of the photon and the position of the maximum of the shower, given by the CES $x$ position.

The measured isolation is corrected to remove the lateral shower and the pile-up contributions. The functional form of the corrections was determined from $W$ and $Z$ data. The current version of the correction is the Run I correction plus a constant, implemented in the CdfEmObject class:

- leakage: $E_{T}^{i s o}=E_{T}^{i s o}-E_{T}\left(c_{1}+c_{2}\right)$ 


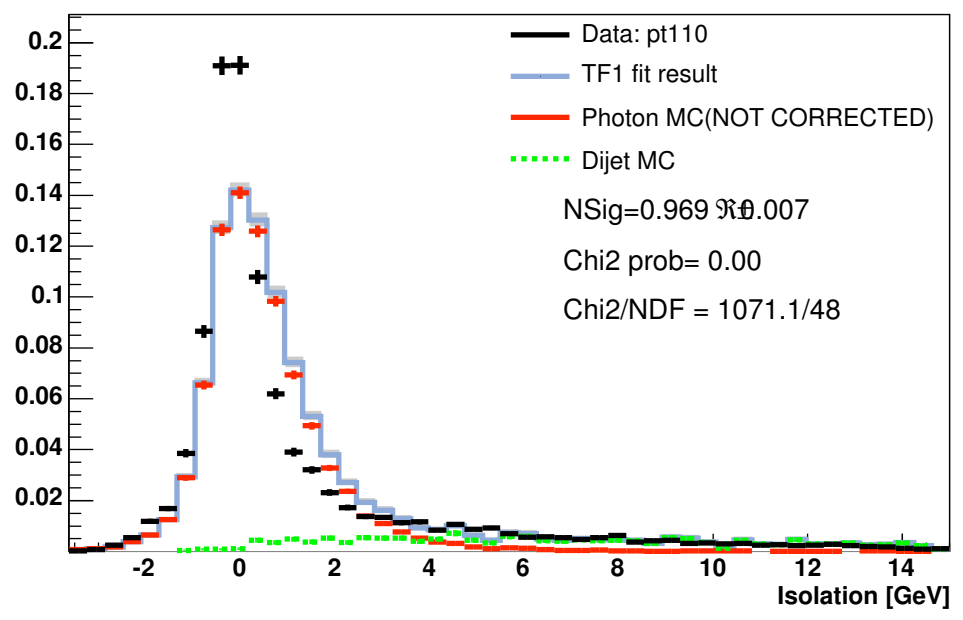

Figure C.1: Fit result for the bin $110<p_{T}<130 \mathrm{GeV}$ using non-corrected photon Monte Carlo templates. The peak in the photon Monte Carlo is wider and shifted to higher isolation values than the data.

$$
\begin{aligned}
& c_{1}=1 /(1.0+0.000280 \exp 0.407(48.4-|x|)) \\
& c_{2}=0.002+0.003\left(E_{T} / 400.0\right)
\end{aligned}
$$

- nver: $E_{T}^{i s o}=E_{T}^{i s o}-0.3563\left(N_{v e r}-1\right)$

The leakage correction is applied first, directly on the measured isolation. The $n$-vertex correction is applied on the leakage-corrected isolation. These corrections are identical for both the data and the Monte Carlo.

To investigate the origin of the discrepancies we see at high $p_{T}$, we study the isolation at raw, leakage and corrected levels in different regions of the (nver,cesx) space in the various photon $p_{T}$ bins of the measurement in both data and Monte Carlo samples (see Section 4.1 for details in the samples). The different regions present different sensitivity to the different components of the isolation energy:

- REGION 1: $n v e r=1, \mid$ ces $x \mid<15 \mathrm{~cm}$

In this region the pile-up contribution is zero and the leakage contribution is very small, so we are especially sensitive to the underlying event.

- REGION 2: nver $>1$, $\mid$ ces $x \mid<15 \mathrm{~cm}$

We use this region to study the effect of the pile-up. 
- REGION 3: $n v e r=1$, $\mid$ ces $x \mid>15 \mathrm{~cm}$

At large $\mid$ ces $x \mid$ the leakage energy is the most important effect. Given that we restrict $n v e r=1$ here, we are not seeing the pile-up effects.

- REGION 4: $n v e r>1, \mid$ ces $x \mid>15 \mathrm{~cm}$

In the region 1, we study the energies in a random cone of $\mathrm{R}=0.4$ situated between 45 and 135 degrees from the photon axis to check the underlying event contribution. These studies are done with the PHOTON_25_ISO trigger dataset and with photon Monte Carlo samples. We find neither the underlying event, the pile-up or the lateral leakage effects can explain the differences in the trend between data and Monte Carlo, and we conclude they can be related to other intrinsic aspects of the simulation, maybe related to soft gluon radiation. The results of these various studies are presented in detail in the sections below. These studies were carried out without having subtracted the background component from the data, of the order of $5 \%$ for the high $p_{T}$. The background contribution to the isolation is therefore very small, and its subtraction is not crucial here, since it is the Monte Carlo which is less isolated than the data.

\section{C.1 Isolation at Raw, Leakage and Corrected levels}

We study the isolation distribution in data and Monte Carlo at raw, leakage and totally corrected levels for the different bins in the photon $p_{T}$. Fig. C.2 shows the raw isolation distribution for the photon bin $150<p_{T}<170 \mathrm{GeV} / \mathrm{c}$ in the region 1 for data and Monte Carlo. In this region the pileup and the leakage contributions to the isolation energy are zero or negligible, and therefore the energy in the isolation cone comes mostly from the underlying event. From this Figure it is clear that even when no correction is applied to the isolation, the photon Monte Carlo predicts wider and higher isolation values than the photon data. This suggests that it could be the underlying event what is causing the shift at high $p_{T}$ (but the random cone studies presented below contradict this hypothesis). Also note the underlying event is not $p_{T}$-dependent but constant with the photon energy, and we observe a $p_{T}$ trend in the Monte Carlo templates ${ }^{1}$.

Fig. C.3 shows the raw isolation for the same $p_{T}$ bin but in the $2^{\text {nd }}$ region. Fig. C.4 shows the fully corrected isolation for the same region. The Monte Carlo and the data present similar differ-

\footnotetext{
${ }^{1}$ Detailed studies on the underlying event at CDF have already shown agreement between the underlying event in data and Monte Carlo using Drell-Yan processes. This studies are described in [61]
} 


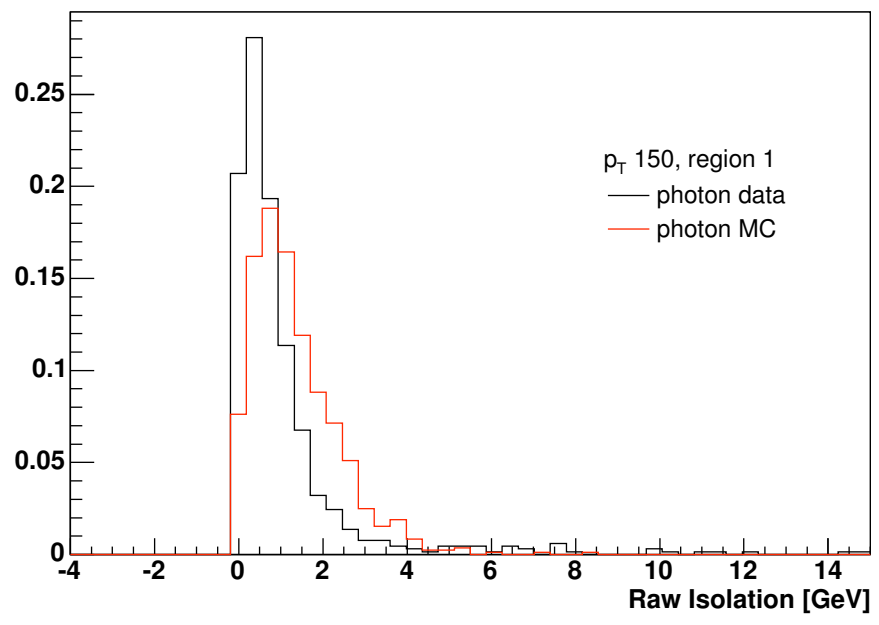

Figure C.2: Data and Monte Carlo raw isolation in the region 1 for $150<p_{T}<170 \mathrm{GeV} / \mathrm{c}$. In this region the pile-up and the leakage contributions to the isolation energy are zero or negligible.

ences than the ones seen at raw level for the region 1 . Therefore, the pile-up is not significantly affecting the discrepancies ${ }^{2}$.

Region 3 is the most sensitive to the leakage contribution, which, contrary to the underlying event and the pile-up, does depend on the photon energy. We have already shown the discrepancies start at raw level and in a region where the leakage effects are minor contributors, but at least this could help to partially explain them. Fig. C.5 shows the raw isolation for data and Monte Carlo in the same $p_{T}$ bin under study. Compared to the raw isolation from other regions (see Fig. C.2 and C.3) it is clear how the energy from the lateral leakage is contributing to the isolation distribution. The data and the Monte Carlo raw isolations present similar behaviour than in the previous regions: the Monte Carlo is wider and shifted to higher isolation values.

Fig. C.6 shows the isolation in the $3^{\text {rd }}$ region after it is corrected for the leakage. Although the raw isolations are not especially different, the same correction is performing differently in data and Monte Carlo. The leakage is not causing the discrepancies, but is clearly contributing to them at large $\mid$ cesx $\mid$.

We run on the Monte Carlo samples requiring $\mid$ ces $x \mid<15 \mathrm{~cm}$ instead of the standard $|\operatorname{ces} x|<21$

\footnotetext{
${ }^{2}$ Note that the fully corrected isolation in region 2 (Fig. C.4) also includes the leakage correction, very small for small $|\operatorname{ces} x|$.
} 


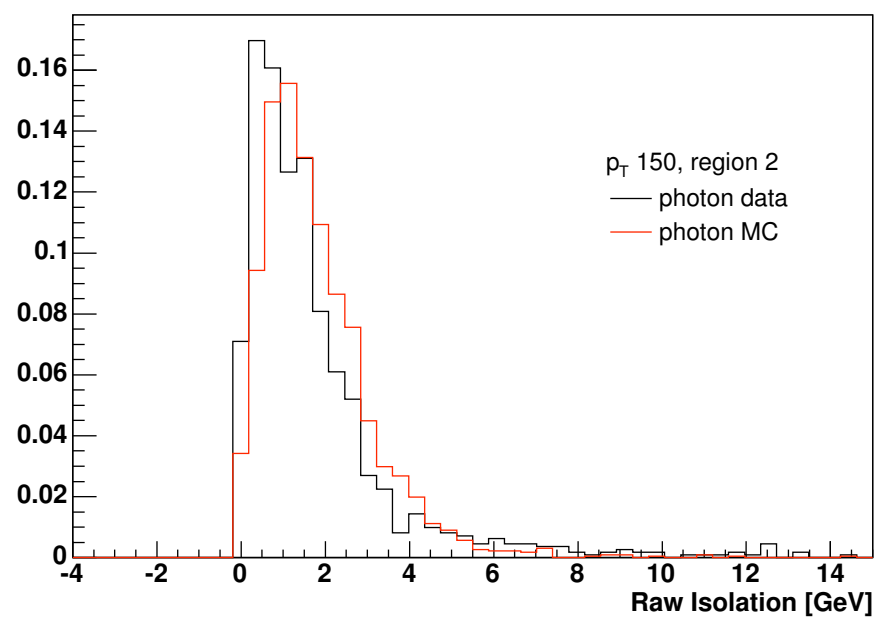

Figure C.3: Data and Monte Carlo raw isolation in the region 2 for $150<p_{T}<170 \mathrm{GeV} / \mathrm{c}$. In this region the pile-up together with the underlying event are the major contributors to the energy in the isolation cone.

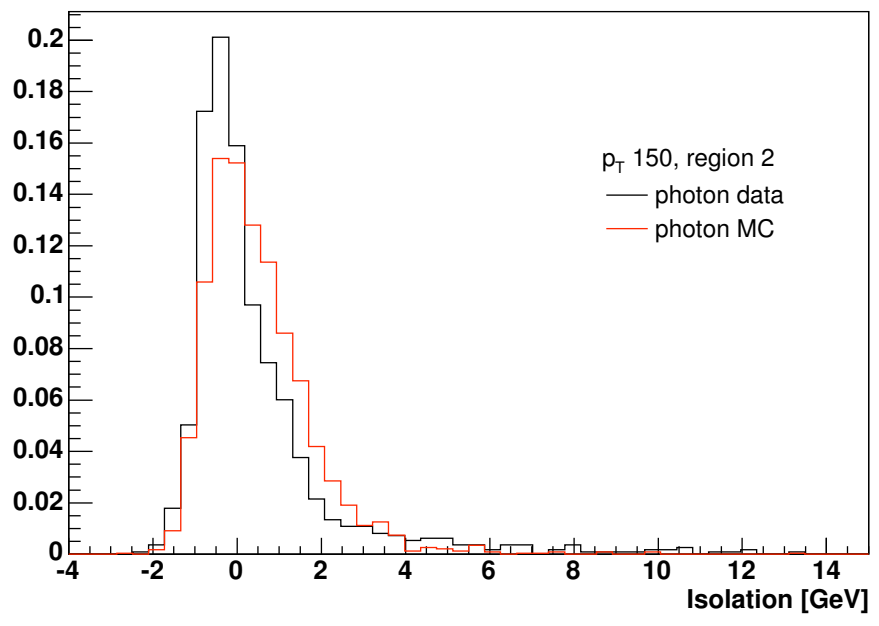

Figure C.4: Data and Monte Carlo corrected isolation in the region 2 for $150<p_{T}<170 \mathrm{GeV} / \mathrm{c}$. After the leakage correction (minor here) and the pile-up correction the discrepancies are not significantly worse than at raw level.

$\mathrm{cm}$ to check if, with this, we can remove the major part of the discrepancies. Fig. C.7 shows the comparison of the fully corrected isolation for data and photon Monte Carlo selected with these 


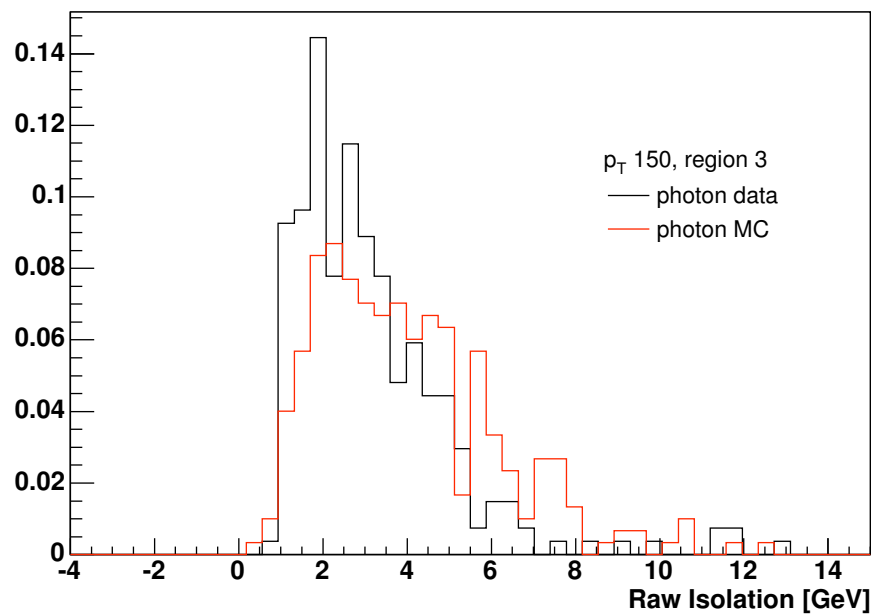

Figure C.5: Data and Monte Carlo raw isolation in the region 3 for $150<p_{T}<170 \mathrm{GeV} / \mathrm{c}$. In this region the leakage contribution dominates.

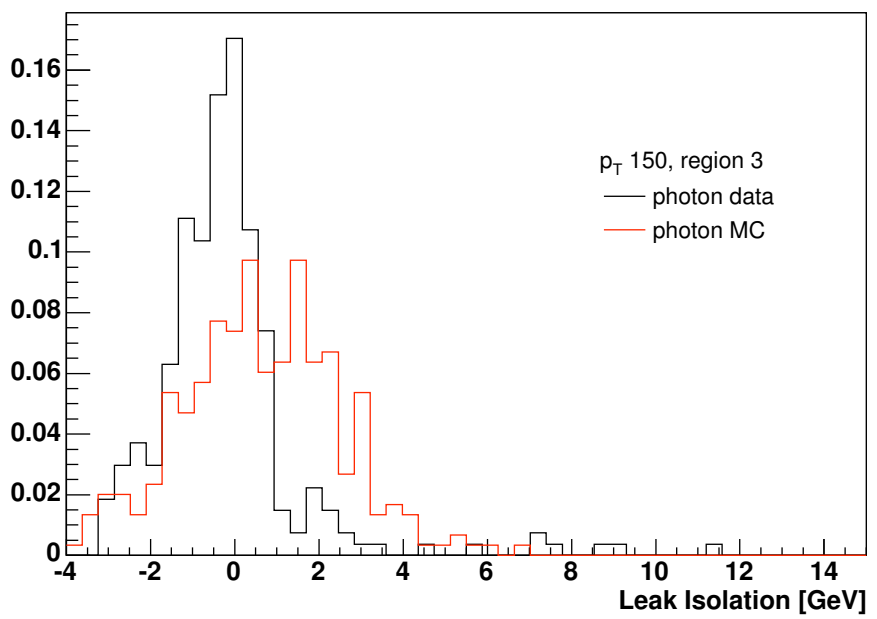

Figure C.6: Data and Monte Carlo leakage isolation in the region 3 for $150<p_{T}<170 \mathrm{GeV} / \mathrm{c}$. After the leakage corrections, differences are larger.

two different requirements for the same bin under study $\left(150<p_{T}<170 \mathrm{GeV} / \mathrm{c}\right)$. The removal of the most sensitive region to the lateral leakage effect does not change significantly the shape of the templates in the Monte Carlo. 


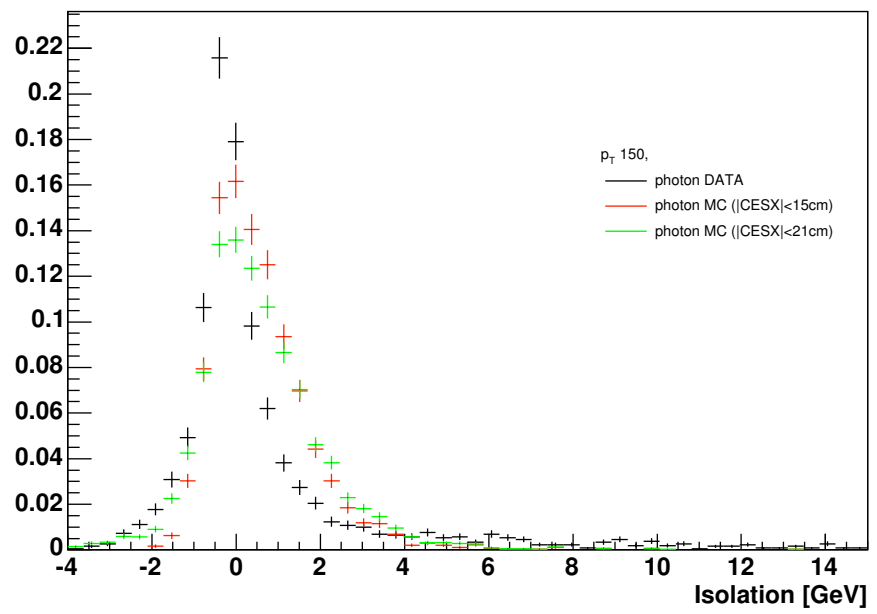

Figure C.7: Data and Monte Carlo corrected isolation for $150<p_{T}<170 \mathrm{GeV} / \mathrm{c}$. Red curve is Monte Carlo selected with $|c e s x|<15 \mathrm{~cm}$. Green curve is Monte Carlo with our nominal cuts and no ad hoc correction.

\section{C.2 Underlying Event study using random cones}

Fig. C.2 in the previous section shows that already the Monte Carlo differs from the data at raw isolation in a region where no pile-up is present and the leakage component is negligible. Therefore, this suggests is the underlying event what could be causing the differences between data and Monte Carlo. To further study the underlying event contribution, we compare the energy in a random cone of $\mathrm{R}=0.4$ between 45 and 135 degrees away from the photon axis. We perform this comparison in the region 1 , where the isolation energy is mainly formed by underlying event energy.

The random cone algorithm is as follows. We randomly select a position, $\hat{p}$, in the CES detector, within the ranges of $\mid$ ces $x \mid<15 \mathrm{~cm}$ and $9<\mid$ cesz $\mid<230 \mathrm{~cm}$. If $\hat{p}$ is far away from the most energetic photon in the event, i.e. the azimuthal angle between $\hat{p}$ and the most energetic photon is within $\pm\left(45^{\circ}-135^{\circ}\right)$, we proceed to calculate the amount of isolation energy $\left(E_{T}^{i s o}\right)$. The $E_{T}^{i s o}$ is the total amount of energy deposited in the calorimeter within a cone of $\mathrm{R}=0.4$ around $\hat{p}$, after subtracting the amount of energy in the seed tower and shoulder towers $\left(E_{E M} \text { and } E_{H A D}\right)^{3}$.

\footnotetext{
${ }^{3}$ The $E_{E M}$ and $E_{H A D}$ include the energies of the seed tower and one shoulder tower $2 / 3$ of the time, and the energies of the seed tower and two shoulder towers $1 / 3$ of the time.
} 
The $E_{H A D}$ is further required to be small ${ }^{4}$. The amount of energy deposited on the CES wires and strips must be smaller than $0.5 \mathrm{GeV}$, respectively. If all criteria are met, we plot $E_{T}^{\text {iso }}$ for the different bins in the photon $p_{T}$.

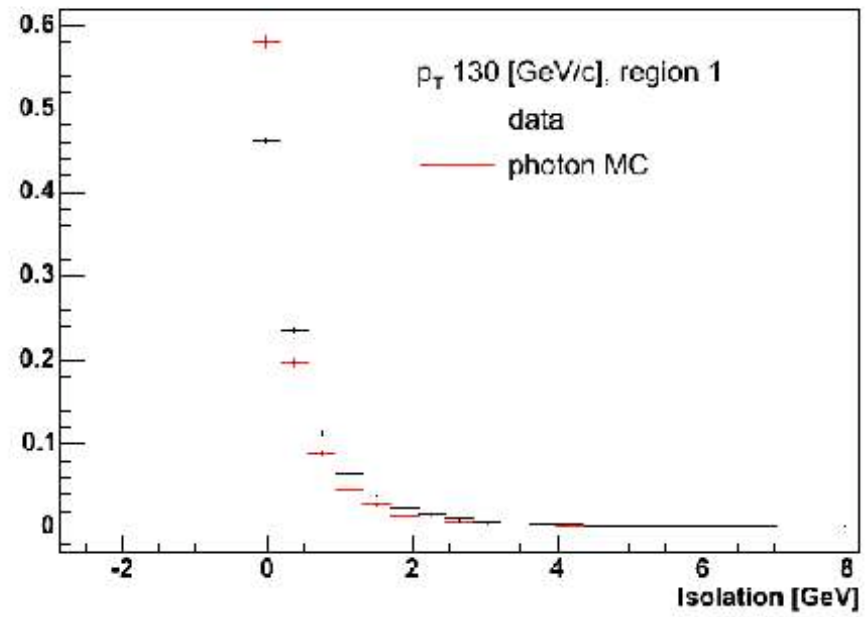

Figure C.8: Energy in the random cone for data (black) and Monte Carlo (red) for $130<p_{T}<150 \mathrm{GeV} / \mathrm{c}$.

Fig. C. 8 shows the energy in the random cone for data and Monte Carlo for $130<p_{T}<150 \mathrm{GeV} / \mathrm{c}$. Contrary to the trend observed in the isolation cone, the energy in the random cone is larger for data than for Monte Carlo. Therefore, the underlying event is not the cause of the discrepancies seen, neither are the corrections applied to the measured isolation.

From the various studies presented in this section, we conclude the discrepancies between data and Monte Carlo cannot be attributed to neither the underlying event, the lateral leakage or the pile-up effects by themselves alone, and that they could be caused by a combination of small effects in all of them or by other effects in the simulation, maybe related to the simulation of the soft radiation in the Monte Carlo.

\footnotetext{
${ }^{4}$ The $E_{H A D}$ is required to be smaller than $0.055 E_{E M}$ for $E_{E M}>25 \mathrm{GeV}$ and 1.375 for $E_{E M}<25 \mathrm{GeV}$.
} 


\section{Appendix D}

\section{Rewighting of the photon Monte Carlo}

The photon Monte Carlo samples used for the unfolding procedure of the cross section were generated with CTEQ5L PDF, which introduces a dependence of the shape of the $p_{T}$ spectrum different to that in the data. In order to avoid any effect in the shape unfolding factors due to the choice of PDFs in the Monte Carlo, the PYTHIA sample is reweighted to follow the prompt photon $p_{T}$ distribution measured from the data. The weights are extracted by fitting the ratio data/Monte Carlo in Fig. D.1. The obtained function is applied event by event in $\hat{p_{T}}$.

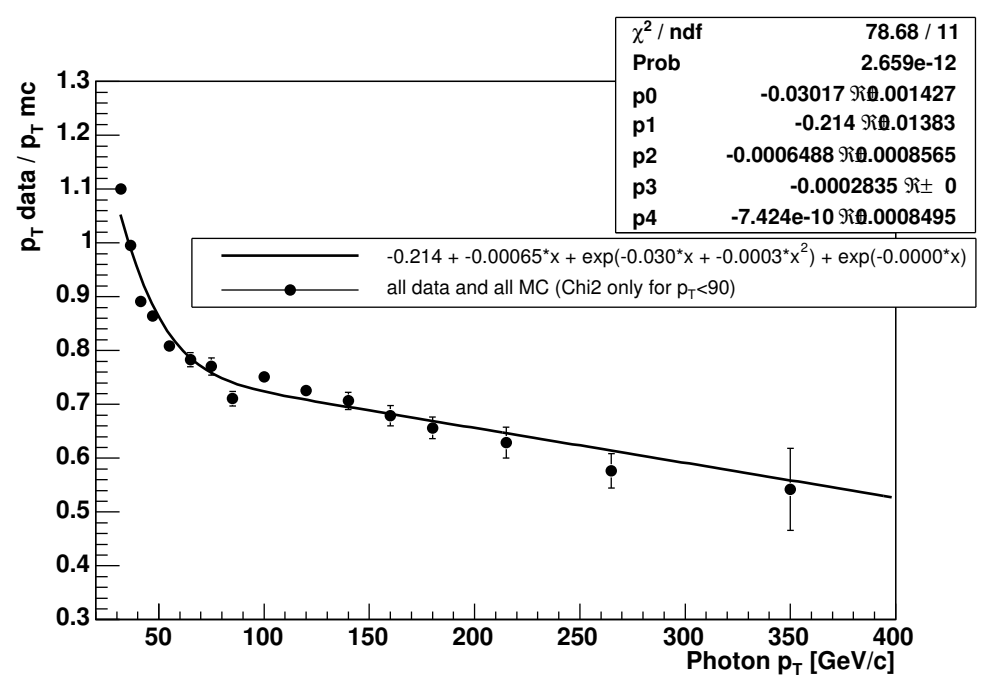

Figure D.1: Data/PYTHIA vs the $p_{T}$ of the photon. 
Fig. D.2 shows the comparison of the photon $p_{T}$ before the Monte Carlo $p_{T}$ spectrum is corrected. Fig. D.3 shows the same comparison after the events in the Monte Carlo are reweighted according to the function in Fig. D.1. After the reweighting procedure, the shapes of the photon $p_{T}$ distributions in data and Monte Carlo agree within $1 \%$. The change in the unfolding factors due to the reweighting is less than $1 \%$ for all the measured range.

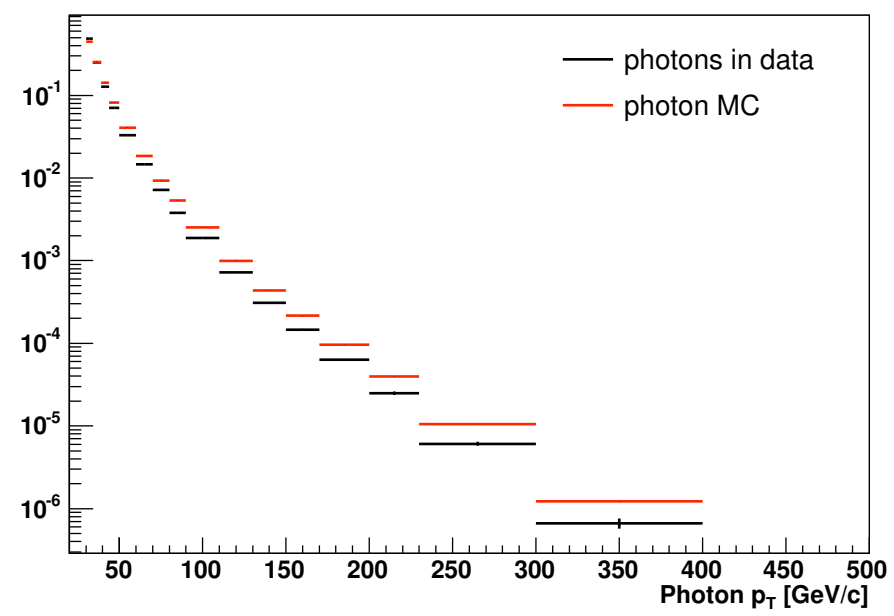

Figure D.2: Comparison of the $p_{T}$ of the photon in data (after the background is subtracted) and Monte Carlo before the reweighting function is applied to the Monte Carlo. The distributions are normalized to their area. 


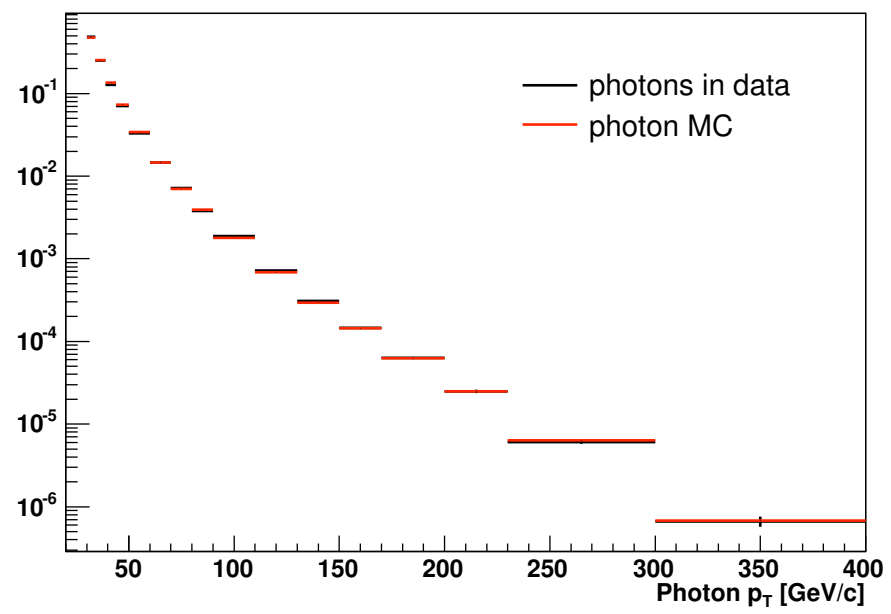

Figure D.3: Comparison of the $p_{T}$ of the photon in data (after the background is subtracted) and Monte Carlo after the reweighting function is applied to the Monte Carlo. The distributions are normalized to their area. 



\section{Bibliography}

[1] D. J. Gross and F. Wilczec, Asymptotically Free Gauge Theories, Phys. Rev. D 8, 3633 (1973).

[2] B. Abbott et al (DO Collaboration), Phys. Rev. Lett. 84, 2786 (2000);

V.M. Abazov et al (DO Collaboration), Phys. Rev. Lett. 87, 251805 (2001);

D. Acosta et al (CDF Collaboration), Phys. Rev. D 65, 112003 (2002).

[3] V.M. Abazov et al (DO Collaboration), Measurement of the Isolated Cross Section in $p \bar{p}$ Collsions at $\sqrt{s}=1.96 \mathrm{TeV}$, Phys. Lett. B 639, 151 (2006).

[4] E. Annassontzis et al. (R806 Collaboration), High $p_{T}$ direct photon production in pp collisions, Z. Phys. C 13277 (1982).

M Bonesini et al. (WA70 Collaboration), Production of high transverse momentum prompt photons and neutral pions in proton-proton interactions at $280 \mathrm{GeV} / \mathrm{c}$, Z. Phys. C 38371 (1988).

C. Alba et al. (UA1 Collaboration), Direct photon production at the CERN proton-antiproton collider, Phys. Lett. B 209385 (1988).

A.L.S. Angelis et al. (R110 Collaboration), Direct photon production at the CERN ISR, Nuc. Phys. B 327541 (1989).

E. Anassontzis et al. (R807/AFS Collaboration), High $p_{T}$ photon and $\pi^{0}$ production, inclusive and with a recoil hadronic jet, in pp collisions at $\sqrt{s}=63 \mathrm{GeV}$, Sov. J. Nuc. Phys. 515 (1990). J. Alliti et al. (UA2 Collaboration), A measurement of the direct photon producution cross section at the CERN pbarpcollider, Phys. Lett. B 263544 (1991).

G. Ballocchi et al. (UA6 Collaboration), Direct photon cross sections in proton-proton and antiproton-proton interactions at $\sqrt{s}=24.3 \mathrm{GeV}$, Phys. Lett. B 436222 (1998).

L. Apanasevich et al. (E706 Collaboration), Evidence for parton $k_{T}$ effects in high $p_{T}$ particle 
production, Phys. Rev. Lett. 812642 (1998).

[5] D. Acosta et al., Phys. Rev. D 65, 112003 (2002);

F. Abe et al., Phys.Rev.D 57, 67-77 (1998);

F. Abe et al., Phys. Rev. Lett. 73, 2662 (1994).

[6] R.K. Ellis, W.J. Stirling and B.R. Webber, $Q C D$ and Collider Physics, Cambridge University Press (1996).

[7] S. Bethke, Experimental Tests of Asymptotic Freedom, arXiv:hep-ex/0606035.

[8] L. N. Lipatov, Sov. J. Nucl. Phys. 20, 95 (1975);

V. N. Gribov and L. N. Lipatov, $e^{+} e^{-}$Pair Annihilation and Deep Inelastic ep Scattering in Perturbation Theory, Sov. J. Nucl. Phys. 15, 438 (1972);

G. Altarelli and G. Parisi, Nucl. Phys. B 126, 298 (1977);

Yu. L. Dokshitzer, Calculation of the Structure Functions for Deep Inelastic Scattering and $e^{+} e^{-}$Annihilation by Perturbation Theory in Quantum Chromodynamics, Sov. Phys. JETP 46, 416 (1977).

[9] CTEQ Webpage, http://www.phys.psu.edu/cteq

Phys. Rev. D 51, 4763-4782 (1995).

[10] S. Alekin, Phys. Rev. D 68, 014002 (2003).

[11] A. D. Martin, R. G. Roberts, W. J. Stirling, and R. S. Thorne, Phys. Lett. B 604, 61 (2004).

[12] J. Pumplin, et al., Uncertainties of Predictions from Parton Distribution Functions II. The Hessian Method, Phys. Rev. D 65, 014013 (2001) [arXiv:hep-ph/0101032].

[13] P. Aurenche, M. Fontannaz, J.P. Guillet and E. Pilon, Cross section of isolated prompt photons in hadron hadron collisions, JHEP 0205, 028 (2002) [arXiv:hep-ph/0204023].

[14] L. Bourhis, M. Fontannaz and J. Ph. Guillet, Quark and Gluon Fragmentation Funtions into photons, Eur. Phys. J. C 2, 529-537 (1998) [arXiv:hep-ph/9704447v1 (1997)].

[15] D. Brusculic et al., ALEPH Collaboration, Z. Phys. C 69, 379 (1996).

[16] S. Abachi et al., HRS Collaboration, Phys. Rev. D 40, 706 (1989). 
[17] H.-L. Lai, H.-N. Li, Origin of the $k_{T}$ smearing in direct photon production, arXiv:hep$\mathrm{ph} / 9802414 \mathrm{v} 1$ (1998).

[18] P. Aurenche, M. Fontannaz, J.P. Guillet, B. Kniehl, E. Pilon and M. Werlen, A critical phenomenological study of inclusive photon production in hadronic collisions, arXiv:hep$\mathrm{ph} / 9811382 \mathrm{v} 1$ (1998).

[19] L. Apanasevich et al., $k_{T}$ Effects in Direct-Photon Production, arXiv:hep-ph/9808467v1 (1998).

[20] J. Huston, private communication.

[21] P. Aurenche, M.Fontanaz, J.P. Guillet, E. Pilon and M. Werlen, A new critical study of photon production in hadronic collisions, Phys. Rev. D 73, 094007 (2006) [arXiv:hep$\mathrm{ph} / 0602133]$.

[22] http://wwwlapp.in2p3.fr/lapth/PHOX_FAMILY/jetphox_soon.html

[23] B. Melle and P. Nason, The Fragmentation Function for Heavy Quarks in QCD, Nucl. Phys. B 361, 626 (1991).

[24] B. Anderson, G. Gustafson, G. Ingelman and T. Sjöstrand, Parton Fragmentation and String Dynamics, Phys. Rev. Lett. 97, 31 (1983);

T. Sjöstrand, Jet Fragmentation of Nearby Partons, Nucl. Phys. B 248, 469 (1984).

[25] T. Sjöstrand et al., PYTHIA 6.4 Physics and Manual, FERMILAB-PUB-06-052-CD-T (2006) [arXiv:hep-ph/0603175].

[26] S. Agostinelli et al., GEANT 4 A Simulation Toolkit, Nuclear Instruments and Methods in Physics Research Section A 506, 3 (2003).

[27] G.A. Schuler and T. Sjöstrand, Phys. Lett. B 300 169, (1993);

G.A. Schuler and T. Sjöstrand, Nucl. Phys. B 407 539, (1993).

[28] C. Friberg and T. Sjöstrand, Eur. Phys. J. C 13 151, (2000); JHEP 09 010, (2000); Phys. Lett. B 492 123, (2000).

[29] T. Affolder et al. (CDF Collaboration), Charged Jet Evolution and the Underlying Event in Proton-Antiproton Collisions at 1.8 TeV, Phys. Rev. D 65, 092002 (2002). 
[30] R. Field, CDF Run II Monte Carlo Tunes, FERMILAB-PUB-06-408-E (2006).

[31] CDF Collaboration, CDF Run II: Technical Design Report, FERMILAB-PUB-96-390-E (1996).

[32] A. Sill, CDF Run II Silicon Tracking Projects, Nucl. Instrum. Meth. A 447, 1 (2000).

[33] T. Affolder et al., Central Outer Tracker, Nucl. Instrum. Meth. A 526, 249 (2004).

[34] D. Acosta et al., A Time-Of-Flight Detector in CDF-II, Nucl. Instrum. Meth. A 518, 605 (2004).

[35] L. Balka et al., The CDF Central Electromagnetic Calorimeter, Nucl. Instrum. Meth. A 267, 272 (1988), FERMILAB-PUB-87-172-E.

[36] S. Bertolucci et al., The CDF Central and Endwall Hadron Calorimeter, Nucl. Instrum. Meth. A 267, 301 (1988), FERMILAB-PUB-87-174-E.

[37] M. Albrow et al., The CDF Plug Upgrade Electromagnetic Calorimeter: Test Beam Results, Nucl. Instrum. Meth. A 480, 524 (2002), FERMILAB-PUB-01-045-E.

[38] S. Lusin, Calibration and Testing of the CDF II Endplug Calorimeter, FERMILAB-CONF98-003-E (1007).

[39] G. Apollinari et al., Shower Maximum Detector for the CDF Plug Upgrade Calorimeter, Nucl. Instrum. Meth. A 412, 512 (1998).

[40] M. Albrow et al., A Preshower Detector for the CDF Plug Upgrade: Test Beam Results, Nucl. Instrum. Meth. A 431, 104 (1999).

[41] C. M. Ginsburg, CDF Run 2 Muon System, Eur. Phys. J. C 33, S1002 (2004), FERMILABCONF-03-386-E.

[42] D. Acosta et al., The Performance of the CDF Luminosity Monitor, Nucl. Instrum. Meth. A 494, 57 (2002).

[43] H.-L. Lai et al (CTEQ), Eur. Phys. J C 12, 375 (2000), [arXiv:hep-ph/9903282].

[44] R. Culbertson, T. Pratt, Search for a High-Mass Diphoton State and Limits on RandallSundrum Gravitons at CDF, CDF/PUB/EXOTIC/PUBLIC/7098;

T. Aaltonen et al. (CDF Collaboration), Phys. Rev. Lett. 99, 171801 (2007). 
[45] M. Goncharov, Calorimeter Timing System at CDF, Calor 2006 conference proceedings, CDF/PUB/CALORIMETRY/PUBLIC/8456.

[46] K. Yasuoka et al., Response maps of the CDF central electromagnetic calorimeter with electrons, Nucl. Instrum. Methods A 267, 315 (1988).

[47] F. Abe et al. (CDF Collaboration), Nucl. Instrum. Methods A 271, 387 (1988).

[48] S. Malik, $W$ mass and width measurements at the Tevatron., CDF/PUB/ELECTROWEAK/PUBLIC/8999, Proceedings for the 15th International Workshop on Deep Inelastic Scattering (DIS 2007).

[49] K. Ikado, A. V. Kotwal, K. Maeshima, Level-2 Trigger and Level-3 Filters for Ultra-High $E_{T}$ Electrons and Photons in Run 2, CDF/DOC/TRIGGER/CDFR/5602.

[50] R. Culbertson, C. Deluca, M. Martinez-Perez, S.-S. Yu (CDF Collaboration), Measurement of the Inclusive Isolated Photon Cross Section at CDF, Proceedings for ICHEP'08, [arXiv:hepex/0810.0553].

[51] M. Goncharov, T. Kamon, S.W. Lee, V. Krutelyov, V. Khotilovich, D. Toback, P. Wagner, H. Frisch, H. Sanders, M. Cordelli, F. Happacher, S. Miscetti, and R. Wagner, The Timing System for the CDF Electromagnetic Calorimeters, Nucl. Instrum. Meth. A 543 (2006), preprint server at physics/0512171.

[52] Yanwen Liu, Xin Wu, R. Culbertson, R. Blair, S. Kuhlmann, J. Huston, Diphoton centralcentral cross-section measurement at CDF Run II, CDF/PHYS/CDF/PUBLIC/6312;

D. Acosta et al., Measurement of the Cross Section for Prompt Diphoton Production at CDF 2, CDF/PUB/JET/PUBLIC/7669.

[53] F. Abe et al. (CDF Collaboration) Phys. Rev. D 48, 2998 (1993).

[54] R. Harris et al., Calibration of CPR conversion probability from $\eta$ and $\pi^{0}$ mesons, CDF/ANAL/JET/CDFR/2318.

[55] J. Alitti et al. (UA2 Collaboration), Phys. Lett. B 263, 544 (1991).

[56] C. Hays, private communication;

J. Lee, $\mathrm{W} Z \mathrm{Z}$ cross section working group. 
[57] T. Binoth et al., Eur. Phys. J. C 16, 311 (2000).

S. Catani et al., JHEP 05, 028 (2002).

[58] D. Stump et al., JHEP 10, 046 (2003).

[59] L. Bourhis, M. Fontannaz, and J. P. Guillet, Eur. Phys. J. C 2, 529 (1998).

[60] J. Alitti et al (UA2 Collaboration), Phys. Lett. B 288, 385 (1992);

D. Acosta et al (CDF Collaboration), Phys. Rev. D 70, 074008 (2004);

V.M. Abazov et al (DO Collaboration), Phys. Lett. B 639, 151 (2006).

[61] D. Kar, R. Field, Using Drell-Yan to Probe the Underlying Event in Run 2 at CDF., CDF/PUB/CDF/PUBLIC/9351. 